\title{
BOULIGAND-LEVENBERG-MARQUARDT ITERATION FOR A NON-SMOOTH ILL-POSED INVERSE PROBLEM*
}

\author{
CHRISTIAN CLASON ${ }^{\dagger}$ AND VU HUU NHU ${ }^{\dagger}$
}

\begin{abstract}
In this paper, we consider a modified Levenberg-Marquardt method for solving an ill-posed inverse problem where the forward mapping is not Gâteaux differentiable. By relaxing the standard assumptions for the classical smooth setting, we derive asymptotic stability estimates which are then used to prove convergence of the proposed method. This method can be applied to an inverse source problem for a non-smooth semilinear elliptic PDE where a Bouligand subdifferential can be used in place of the non-existing Fréchet derivative, and we show that the corresponding Bouligand-Levenberg-Marquardt iteration is an iterative regularization scheme. Numerical examples illustrate the advantage over the corresponding Bouligand-Landweber iteration.
\end{abstract}

Key words. inverse problem, iterative regularization, Levenberg-Marquardt method, non-smooth equation

AMS subject classifications. 49K20, 49K40, 90C 31

1. Introduction. We consider an inverse problem of the form

$$
F(u)=y^{\delta},
$$

where $F: D(F) \subset U \rightarrow Y$ is a non-smooth (i.e., not necessarily Gâteaux differentiable) nonlinear operator between Hilbert spaces and the available data $y^{\delta}$ are some approximations of the corresponding true data $y^{\dagger}:=F\left(u^{\dagger}\right)$. Furthermore, $D(F)$ denotes the domain of $F$ and $u^{\dagger}$ is the unknown true solution that needs to be reconstructed.

A typical example of problem (1.1) is the case where $U=Y:=L^{2}(\Omega)$ and $F$ is the solution operator of the non-smooth semilinear elliptic equation

$$
-\Delta y+\max (0, y)=u \quad \text { in } \Omega, \quad y \in H_{0}^{1}(\Omega),
$$

with $u \in L^{2}(\Omega)$ and a bounded domain $\Omega \subset \mathbb{R}^{d}, d \in\{2,3\}$. In this case, $F$ is not Gâteaux differentiable at $u^{\dagger}$ if the set of $x \in \Omega$ such that $y^{\dagger}(x)=0$ has positive measure; see [3, Prop. 3.4]. Moreover, $F$ is completely continuous (see [3, Lem. 3.2]), and (1.1) is therefore ill-posed in the sense that the solution to (1.1) does not depend continuously on the data. A stable solution of (1.1) thus needs regularization techniques. Here we consider iterative regularization techniques, which construct a sequence $\left\{u_{n}^{\delta}\right\}$ of approximations to $u^{\dagger}$ and ensure stability by early stopping at an iteration index $N\left(\delta, y^{\delta}\right)$ chosen, e.g., according to Morozov's discrepancy principle; see, e.g., [4, 15]. Iterative methods have the advantage over variational methods such as Tikhonov regularization that the selection of the regularization parameter (in this case, the stopping index) is part of the method and does not have to be performed by, e.g., checking a sequence of candidates or using additional information on the smoothness of the forward operator that is often not available. An iterative regularization method for (1.2) of Landweber type (which can be interpreted as a generalized gradient descent) was proposed and analyzed in [3]. However, like any first-order scheme, it usually requires a large number of iterations to satisfy the discrepancy principle, especially for small noise. This motivates considering iterative regularization methods of Newton type.

Recall that the Newton method for the smooth version of (1.1) with a continuously Fréchet differentiable operator $F$ reads as

$$
F^{\prime}\left(u_{n}^{\delta}\right)\left(u_{n+1}^{\delta}-u_{n}^{\delta}\right)=y^{\delta}-F\left(u_{n}^{\delta}\right),
$$

*Received February 28, 2019. Accepted July 23, 2019. Published online on September 10, 2019. Recommended by Stefan Kindermann.

${ }^{\dagger}$ Faculty of Mathematics, University of Duisburg-Essen, Thea-Leymann-Strasse 9, 45127 Essen, Germany ( christian.clason, huu.vu\}@uni-due.de). 


\section{ETNA}

Kent State University and

Johann Radon Institute (RICAM)

where $F^{\prime}(u): U \rightarrow Y$ denotes the Gâteaux derivative of $F$ at $u \in D(F)$. However, if (1.1) is ill-posed, then this equation is in general ill-posed as well and needs to be regularized. Applying Tikhonov regularization leads to the Levenberg-Marquardt method

$$
u_{n+1}^{\delta}=\operatorname{argmin}_{u \in D(F)}\left\|F^{\prime}\left(u_{n}^{\delta}\right)\left(u-u_{n}^{\delta}\right)-y^{\delta}-F\left(u_{n}^{\delta}\right)\right\|_{Y}^{2}+\alpha_{n}\left\|u-u_{n}^{\delta}\right\|_{U}^{2}
$$

or, equivalently,

$$
u_{n+1}^{\delta}=u_{n}^{\delta}+\left(\alpha_{n} I+F^{\prime}\left(u_{n}^{\delta}\right)^{*} F^{\prime}\left(u_{n}^{\delta}\right)\right)^{-1} F^{\prime}\left(u_{n}^{\delta}\right)^{*}\left(y^{\delta}-F\left(u_{n}^{\delta}\right)\right),
$$

where $\alpha_{n}>0$ is the Tikhonov parameter. For a linear operator, this method coincides with the non-stationary iterated Tikhonov method studied in, e.g., [1, 7]. As noted above, for noisy data the iteration has to be terminated at a stopping index $N_{\delta}:=N\left(\delta, y^{\delta}\right)<\infty$ in order to be stable. Assuming that $\left\|y^{\delta}-y^{\dagger}\right\|_{Y} \leq \delta$ and that the Tikhonov parameters $\alpha_{n}$ are chosen via a Morozov discrepancy principle, in [6] the regularization property $u_{N_{\delta}}^{\delta} \rightarrow u^{\dagger}$ as $\delta \rightarrow 0$ as well as the logarithmic estimate

$$
N_{\delta}=O(1+|\log (\delta)|)
$$

is shown provided that

$$
\left\|F\left(u_{1}\right)-F\left(u_{2}\right)-F^{\prime}\left(u_{2}\right)\left(u_{1}-u_{2}\right)\right\|_{Y} \leq c\left\|u_{1}-u_{2}\right\|_{U}\left\|F\left(u_{1}\right)-F\left(u_{2}\right)\right\|_{Y}
$$

for all $u_{1}, u_{2} \in \bar{B}_{U}\left(u^{\dagger}, \rho\right)$ and for some constants $c, \rho>0$. In [8], the regularization property as well as the logarithmic estimate (1.5) of the Levenberg-Marquardt method was verified under the a priori choice

$$
\alpha_{n}=\alpha_{0} r^{n}, \quad n=0,1, \ldots,
$$

with $\alpha_{0}>0$ and $r \in(0,1)$ and under the assumption that for any $u_{1}, u_{2} \in \bar{B}_{U}\left(u^{\dagger}, \rho\right)$, there exists a bounded linear operator $Q\left(u_{1}, u_{2}\right): Y \rightarrow Y$ satisfying

$$
F^{\prime}\left(u_{1}\right)=Q\left(u_{1}, u_{2}\right) F^{\prime}\left(u_{2}\right) \quad \text { and } \quad\left\|I-Q\left(u_{1}, u_{2}\right)\right\|_{\mathbb{L}(Y)} \leq L\left\|u_{1}-u_{2}\right\|_{U}
$$

for some constant $L>0$. It is noted that the convergence analysis in $[6,8]$ requires the stability of the method, that is, it holds that

$$
u_{n}^{\delta} \rightarrow u_{n} \quad \text { as } \delta \rightarrow 0 \quad \text { for all } n \leq N\left(\delta, y^{\delta}\right)
$$

with $\delta$ small enough, where $u_{n}^{\delta}$ and $u_{n}$ are generated by the method corresponding to the noisy $(\delta>0)$ and the noise-free $(\delta=0)$ situations, respectively. The continuity of the derivative $F^{\prime}$ (or more specifically, of the linear operator at the right-hand side of (1.4)) with respect to $u$ is therefore essential.

The purpose of this work is to present a modified Levenberg-Marquardt method for solving (1.1) in the spirit of [3,24], where we replace the-possibly nonexistent—Fréchet derivative $F^{\prime}(u)$ in (1.4) by another suitable bounded linear operator $G_{u}$. Our main aim is to show the regularization property of the proposed algorithm under the choice (1.7) of Tikhonov parameters and conditions that relax (1.6) and (1.8). We also prove the logarithmic estimate (1.5) of the stopping index. However, unlike the situation in $[6,8]$, we lack the continuity of the mapping $D(F) \ni u \mapsto G_{u} \in \mathbb{L}(U, Y)$. To overcome this essential difficulty, we shall combine a technique from [8] with the approach in [3] to prove asymptotic stability estimates of the iterates $u_{n}^{\delta}$; see Section 2.3 and Proposition 2.19 in place of the missing 
stability of the method. The proposed method is then applied to a non-smooth ill-posed inverse problem where the forward operator is the solution mapping of (1.2). In this case, the operator $G_{u}$ can be taken from the Bouligand subdifferential of the forward mapping and explicitly characterized by the solution of a suitable linearized PDE; see Proposition 3.1 below. We refer to this special case of the modified Levenberg-Marquardt method as Bouligand-LevenbergMarquardt iteration.

Let us briefly comment on related literature. Newton-type methods and in particular the Levenberg-Marquardt method for approximately solving smooth nonlinear ill-posed problems have been extensively investigated in Hilbert spaces; see, e.g., [4, 6, 15, 18, 21, 22, 23, 25] and the references therein. More recently, inverse problems in Banach spaces have attracted increasing attention, and corresponding iterative regularization methods of Newton type have been developed, e.g., in [9, 10,12, 13, 14, 16, 19, 20]. Considering (1.3) in Banach spaces (in particular, $L^{1}$ or the space of functions of bounded variation) or including additional constraints can lead to non-smooth optimization problems; however, none of the works so far has focused on inverse problems for non-smooth forward operators.

Organization. This paper is organized as follows. After briefly summarizing basic notation, we present the convergence analysis of the modified Levenberg-Marquardt method in Section 2: Section 2.1 is devoted to its well-posedness and the logarithmic estimate of the stopping index $N_{\delta}$; in Section 2.2, we prove its convergence in the noise-free case; in Section 2.3 we verify its asymptotic stability estimates, which are crucial for the proof of the regularization property of the iterative method in Section 2.4. Section 3 introduces an application of the modified Levenberg-Marquardt method to the non-smooth ill-posed inverse source problem for (1.2). Finally, some numerical examples are provided in Section 4.

Notation. For a Hilbert space $X$, we denote by $(\cdot, \cdot)_{X}$ and $\|\cdot\|_{X}$, respectively, the inner product and the norm on $X$. For a given $z$ belonging to a Banach space $Z$ and $\rho>0$, by $B_{Z}(z, \rho)$ and $\bar{B}_{Z}(z, \rho)$ we denote, respectively, the open and closed balls in $Z$ of radius $\rho$ centered at $z$. For each measurable function $u$ on $\Omega$ and a subset $T \subset \mathbb{R}$, the notation $\{u \in T\}$ stands for the sets of almost every $x \in \Omega$ at which $u(x) \in T$. Similarly, given measurable functions $u, v$ on $\Omega$ and subsets $T_{1}, T_{2} \subset \mathbb{R}$, we denote the set of a.e. $x \in \Omega$ such that $u(x) \in T_{1}$ and $v(x) \in T_{2}$ by $\left\{u \in T_{1}, v \in T_{2}\right\}$. For a measurable set $S$ in $\mathbb{R}^{d}$, we write $|S|$ for the $d$-dimensional Lebesgue measure of $S$ and denote by $\chi_{S}$ the characteristic function of the set $S$, i.e., $\chi_{S}(s)=1$ if $s \in S$ and $\chi_{S}(s)=0$ if $s \notin S$. The adjoint operator, the null space, and the range of a linear operator $G$ will be denoted by $G^{*}, \mathcal{N}(G)$, and $\mathcal{R}(G)$, respectively. Finally, we denote by $\mathbb{L}(X)$ and $\mathbb{L}(X, Y)$ the set of all bounded linear operators from a Hilbert space $X$ to itself and from $X$ to another Hilbert space $Y$, respectively.

2. A modified Levenberg-Marquardt method. Let $U$ and $Y$ be real Hilbert spaces and $F$ a non-smooth mapping from $U$ to $Y$ with its domain $D(F) \subset U$. We consider the non-smooth ill-posed problem

$$
F(u)=y^{\delta}
$$

where the noisy data $y^{\delta}$ satisfy

$$
\left\|y^{\delta}-y^{\dagger}\right\|_{Y} \leq \delta
$$

with $y^{\dagger} \in \mathcal{R}(F)$. From now on, let $u^{\dagger}$ be an arbitrary but fixed solution of (2.1) corresponding to the exact data $y^{\dagger}$. For a given number $\rho>0$, we denote by $S_{\rho}\left(u^{\dagger}\right)$ the set of all solutions in $\bar{B}_{U}\left(u^{\dagger}, \rho\right)$ of (2.1) corresponding to the exact data, that is,

$$
S_{\rho}\left(u^{\dagger}\right):=\left\{u^{*} \in D(F): F\left(u^{*}\right)=y^{\dagger},\left\|u^{*}-u^{\dagger}\right\|_{U} \leq \rho\right\} .
$$


Throughout this work, we make the following assumptions on $F$ :

(A1) There exists a constant $\rho_{0}>0$ such that $\bar{B}_{U}\left(u^{\dagger}, \rho_{0}\right) \subset D(F)$. Furthermore, there exists a family of bounded linear operators $\left\{G_{u}: u \in \bar{B}_{U}\left(u^{\dagger}, \rho\right)\right\} \subset \mathbb{L}(U, Y)$ such that for all $\rho \in\left(0, \rho_{0}\right]$ and $u, \hat{u}$ in $\bar{B}_{U}\left(u^{\dagger}, \rho\right)$, the generalized tangential cone condition

(GTCC)

$$
\left\|F(\hat{u})-F(u)-G_{u}(\hat{u}-u)\right\|_{Y} \leq \eta(\rho)\|F(\hat{u})-F(u)\|_{Y}
$$

holds for some non-decreasing function $\eta:\left(0, \rho_{0}\right] \rightarrow(0, \infty)$ satisfying

$$
\eta_{0}:=\eta\left(\rho_{0}\right)<1 \text {. }
$$

Moreover, for any pair $u_{1}, u_{2} \in \bar{B}_{U}\left(u^{\dagger}, \rho_{0}\right)$, there exists a bounded linear operator $Q\left(u_{1}, u_{2}\right) \in \mathbb{L}(Y)$ such that

$$
G_{u_{1}}=Q\left(u_{1}, u_{2}\right) G_{u_{2}}
$$

and that for all $\rho \in\left(0, \rho_{0}\right]$,

$$
\|I-Q(u, \hat{u})\|_{\mathbb{L}(Y)} \leq \kappa(\rho) \quad \text { for all } u, \hat{u} \in \bar{B}_{U}\left(u^{\dagger}, \rho\right)
$$

and for some non-negative and non-decreasing function $\kappa$ on the interval $\left(0, \rho_{0}\right]$ with $\kappa_{0}:=\kappa\left(\rho_{0}\right)$.

(A2) The operator $G_{u^{+}}: U \rightarrow Y$ is compact.

We will furthermore require that $\kappa(\rho)$ and $\eta(\rho)$ can be made sufficiently small by choosing $\rho$ small enough; we will make this more precise during the analysis in this section. In Section 3.2, we will verify that this is possible for the Bouligand-Levenberg-Marquardt method applied to the non-smooth PDE (1.2).

REMARK 2.1. If (GTCC) is valid, then for all $\rho \in\left(0, \rho_{0}\right]$ and $u, \hat{u} \in \bar{B}_{U}\left(u^{\dagger}, \rho\right)$, it holds that

$$
\left\|F(\hat{u})-F(u)-G_{u}(\hat{u}-u)\right\|_{Y} \leq \frac{\eta(\rho)}{1-\eta_{0}}\left\|G_{u}(\hat{u}-u)\right\|_{Y}
$$

and

$$
\|F(\hat{u})-F(u)\|_{Y} \leq \frac{1}{1-\eta_{0}}\left\|G_{u}(\hat{u}-u)\right\|_{Y}
$$

where the last inequality leads to continuity of $F$ at $u$ and hence on $\bar{B}_{U}\left(u^{\dagger}, \rho\right)$. Moreover, if (2.4) holds, then it holds that

$$
\left\|Q\left(u_{1}, u_{2}\right)\right\|_{\mathbb{L}(Y)} \leq \kappa_{0}+1
$$

for all $u_{1}, u_{2} \in \bar{B}_{U}\left(u^{\dagger}, \rho_{0}\right)$. In addition, if Assumptions (A1) and (A2) are satisfied, then all of the Assumptions (A1) and (A2) are fulfilled with $F$ and $G_{u}$ replaced, respectively, by $t F$ and $t G_{u}$ for some positive number $t$ small enough.

We shall consider the modified Levenberg-Marquardt method defined as

$$
u_{n+1}^{\delta}=u_{n}^{\delta}+\left(\alpha_{n} I+G_{u_{n}^{\delta}}^{*} G_{u_{n}^{\delta}}\right)^{-1} G_{u_{n}^{\delta}}^{*}\left(y^{\delta}-F\left(u_{n}^{\delta}\right)\right), \quad n=0,1, \ldots,
$$

where $u_{0}^{\delta}:=u_{0}$ and $\left\{\alpha_{n}\right\}$ is given by

$$
\alpha_{n}=\alpha_{0} r^{n}, \quad n=0,1, \ldots
$$


for some constants $\alpha_{0}>0$ and $r \in(0,1)$. We will assume that $\alpha_{0}$ is chosen such that

$$
\left\|G_{u^{\dagger}}\right\|_{\mathbb{L}(U, Y)} \leq \alpha_{0}^{1 / 2} .
$$

Note that according to Remark 2.1, this condition can be enforced for any $\alpha_{0}>0$ by scaling the problem (1.1) as well as $G_{u}$ accordingly.

The iteration is terminated via the discrepancy principle

$$
\left\|y^{\delta}-F\left(u_{N_{\delta}}^{\delta}\right)\right\|_{Y} \leq \tau \delta<\left\|y^{\delta}-F\left(u_{n}^{\delta}\right)\right\|_{Y} \quad \text { for all } 0 \leq n<N_{\delta},
$$

where $\tau>1$ is a given number. Here $N_{\delta}:=N\left(\delta, y^{\delta}\right)$ stands for the stopping index of the iterative method.

By $\left\{u_{n}\right\}$, we denote the sequence of iterates defined by (2.8) corresponding to the noisefree case $(\delta=0)$, i.e.,

$$
u_{n+1}=u_{n}+\left(\alpha_{n} I+G_{u_{n}}^{*} G_{u_{n}}\right)^{-1} G_{u_{n}}^{*}\left(y^{\dagger}-F\left(u_{n}\right)\right), \quad n=0,1, \ldots
$$

For the ease of exposition, from now on, we use the notations

$$
\begin{aligned}
& e_{n}^{\delta}:=u_{n}^{\delta}-u^{\dagger}, \quad G_{n}^{\delta}:=G_{u_{n}^{\delta}}, \quad A_{n}^{\delta}:=G_{n}^{\delta *} G_{n}^{\delta}, \quad B_{n}^{\delta}:=G_{n}^{\delta} G_{n}^{\delta *}, \\
& e_{n}:=u_{n}-u^{\dagger}, \quad G_{n}:=G_{u_{n}}, \quad A_{n}:=G_{n}^{*} G_{n}, \quad B_{n}:=G_{n} G_{n}^{*}, \\
& G_{\dagger}:=G_{u^{\dagger}}, \quad A:=G_{\dagger}^{*} G_{\dagger}, \quad B:=G_{\dagger} G_{\dagger}^{*} .
\end{aligned}
$$

REMARK 2.2. If $F$ is smooth and if $G_{u}=F^{\prime}(u)$, then (2.8) coincides with the classical Levenberg-Marquardt method; see, e.g., [6, 8, 15, 25].

2.1. Well-posedness. We first show the well-posedness of the proposed iterative method as well as the logarithmic estimate of the stopping index $N_{\delta}$.

The first lemma gives a useful tool to estimate the difference between the iterates.

LEMMA 2.3 (cf. [8, Lem. 2]). Assume that (2.3) and (2.4) are fulfilled. Let $\rho \in\left(0, \rho_{0}\right]$. For any $u, v \in \bar{B}_{U}\left(u^{\dagger}, \rho\right)$, let $A_{u}:=G_{u}^{*} G_{u}$ and $A_{v}:=G_{v}^{*} G_{v}$. Then for any $\alpha>0$, the following identities hold

$$
\left(\alpha I+A_{u}\right)^{-1}-\left(\alpha I+A_{v}\right)^{-1}=\left(\alpha I+A_{v}\right)^{-1} G_{v}^{*} R_{\alpha}(u, v)
$$

and

$$
\left(\alpha I+A_{u}\right)^{-1} G_{u}^{*}-\left(\alpha I+A_{v}\right)^{-1} G_{v}^{*}=\left(\alpha I+A_{v}\right)^{-1} G_{v}^{*} S_{\alpha}(u, v),
$$

where $R_{\alpha}(u, v): U \rightarrow Y$ and $S_{\alpha}(u, v): Y \rightarrow Y$ are bounded linear operators satisfying

$$
\left\|R_{\alpha}(u, v)\right\|_{\mathbb{L}(U, Y)} \leq \alpha^{-1 / 2} \kappa(\rho) \quad \text { and } \quad\left\|S_{\alpha}(u, v)\right\|_{\mathbb{L}(Y)} \leq 3 \kappa(\rho) .
$$

Proof. The proof is analogous to that in [8]. We see from (2.3) that

$$
\begin{aligned}
\left(\alpha I+A_{u}\right)^{-1}-\left(\alpha I+A_{v}\right)^{-1} & \\
& =\left(\alpha I+A_{v}\right)^{-1}\left(A_{v}-A_{u}\right)\left(\alpha I+A_{u}\right)^{-1} \\
& =\left(\alpha I+A_{v}\right)^{-1}\left(G_{v}^{*}\left(G_{v}-G_{u}\right)+\left(G_{v}^{*}-G_{u}^{*}\right) G_{u}\right)\left(\alpha I+A_{u}\right)^{-1} \\
& =\left(\alpha I+A_{v}\right)^{-1}\left(G_{v}^{*}(Q(v, u)-I) G_{u}+G_{v}^{*}\left(I-Q(u, v)^{*}\right) G_{u}\right)\left(\alpha I+A_{u}\right)^{-1} \\
& =\left(\alpha I+A_{v}\right)^{-1} G_{v}^{*}\left((Q(v, u)-I)+\left(I-Q(u, v)^{*}\right)\right) G_{u}\left(\alpha I+A_{u}\right)^{-1} \\
& =\left(\alpha I+A_{v}\right)^{-1} G_{v}^{*} R_{\alpha}(u, v)
\end{aligned}
$$


with

$$
R_{\alpha}(u, v):=\left((Q(v, u)-I)+\left(I-Q(u, v)^{*}\right)\right) G_{u}\left(\alpha I+A_{u}\right)^{-1} .
$$

Easily, (2.4) and Lemma A.4 ensure that $R_{\alpha}(u, v)$ satisfies the first inequality in (2.13). On the other hand, using (2.3) and the above representation yields

$$
\begin{aligned}
(\alpha I & \left.+A_{u}\right)^{-1} G_{u}^{*}-\left(\alpha I+A_{v}\right)^{-1} G_{v}^{*} \\
& =\left(\alpha I+A_{v}\right)^{-1}\left(G_{u}^{*}-G_{v}^{*}\right)+\left[\left(\alpha I+A_{u}\right)^{-1}-\left(\alpha I+A_{v}\right)^{-1}\right] G_{u}^{*} \\
& =\left(\alpha I+A_{v}\right)^{-1} G_{v}^{*}\left(Q(u, v)^{*}-I\right)+\left(\alpha I+A_{v}\right)^{-1} G_{v}^{*} R_{\alpha}(u, v) G_{u}^{*} \\
& =\left(\alpha I+A_{v}\right)^{-1} G_{v}^{*} S_{\alpha}(u, v)
\end{aligned}
$$

with

$$
\begin{aligned}
S_{\alpha}(u, v) & :=Q(u, v)^{*}-I+R_{\alpha}(u, v) G_{u}^{*} \\
& =Q(u, v)^{*}-I+\left((Q(v, u)-I)+\left(I-Q(u, v)^{*}\right)\right)\left(\alpha I+B_{u}\right)^{-1} B_{u}
\end{aligned}
$$

and $B_{u}:=G_{u} G_{u}^{*}$. From the definition of $S_{\alpha},(2.4)$ and Lemma A.3 lead to the second inequality in (2.13).

To simplify the notation in the following proofs, we introduce the constants

$$
c_{0}:=\frac{1}{\sqrt{r}}, \quad c_{1}:=\frac{1}{1-\sqrt{r}}, \quad c_{2}:=\frac{1}{\sqrt{r}(1-\sqrt{r})}, \quad c_{3}:=\frac{\sqrt{r}}{1-r}, \quad c_{4}:=\frac{1}{1-r},
$$

as well as

$$
K_{0}(r, \nu):=\frac{1}{\sqrt{r}\left(r^{\nu-1 / 2}-1\right)}, \quad K_{1}(r, \nu):=\frac{1}{r\left(r^{\nu-1 / 2}-1\right)}
$$

for $0 \leq \nu<\frac{1}{2}$.

Let now $\tilde{N}_{\delta} \in \mathbb{N}$ be such that

$$
\alpha_{\tilde{N}_{\delta}} \leq\left(\frac{\delta}{\gamma_{0}\left\|e_{0}\right\|_{U}}\right)^{2}<\alpha_{n}, \quad \text { for all } 0 \leq n<\tilde{N}_{\delta},
$$

for a constant

$$
\gamma_{0}>\frac{2 c_{0}}{\left(1-\eta_{0}\right)\left(\tau-\tau_{0}\right)}
$$

with $\tau>\tau_{0}>1$. We can now prove a logarithmic estimate for $\tilde{N}_{\delta}$, which will later be used to obtain the corresponding estimate for the actual stopping index $N_{\delta}$.

LEMMA 2.4. Let $\tilde{N}_{\delta}$ be defined by (2.16). Then it holds that

$$
\tilde{N}_{\delta}=O(1+|\log (\delta)|) .
$$

Proof. From (2.16) and (2.9), we conclude that

$$
\left(\frac{\delta}{\gamma_{0}\left\|e_{0}\right\|_{U}}\right)^{2}<\alpha_{0} r^{\tilde{N}_{\delta}-1}
$$


which, together with the fact that $0<r<1$, directly gives

$$
\tilde{N}_{\delta}<1+2 \log _{r} \delta-2 \log _{r}\left(\gamma_{0}\left\|e_{0}\right\|_{U}\right)-\log _{r} \alpha_{0}
$$

and hence the desired estimate.

We now show a uniform bound for the iterates and the error by, if necessary, further restricting the radius $\rho$ of the neighborhood of $u^{\dagger}$.

LEMMA 2.5 (cf. [8, Lem. 4]). Let $\left\{\alpha_{n}\right\}$ be defined by (2.9) and (2.10). Assume that Assumption (A1) holds. Assume further that there exists a positive constant $\rho_{1} \leq \rho_{0}$ such that

$$
\left\{\begin{aligned}
2 \kappa\left(\rho_{1}\right)+\frac{c_{0}}{1-\eta_{0}}\left(1+3 \kappa_{0}\right) \eta\left(\rho_{1}\right) & \leq \frac{c_{0}}{2 c_{2}}, \\
\left(c_{1}+3\right) \kappa\left(\rho_{1}\right)+\frac{2 c_{2}}{1-\eta_{0}}\left(1+3 \kappa_{0}\right) \eta\left(\rho_{1}\right) & \leq 1 .
\end{aligned}\right.
$$

Let $\rho \in\left(0, \rho_{1}\right]$ be arbitrary, and let $u_{0} \in U$ be such that $\left(2+c_{1} \gamma_{0}\right)\left\|e_{0}\right\|_{U}<\rho$. Then it holds that

(i) $u_{n}^{\delta} \in \bar{B}_{U}\left(u^{\dagger}, \rho\right)$;

(ii) $\left\|e_{n}^{\delta}\right\|_{U} \leq\left(2+c_{1} \gamma_{0}\right)\left\|e_{0}\right\|_{U}<\rho$;

(iii) $\left\|G_{\dagger} e_{n}^{\delta}\right\|_{Y} \leq\left(c_{0}+2 c_{2} \gamma_{0}\right)\left\|e_{0}\right\|_{U} \alpha_{n}^{1 / 2}$

for all $0 \leq n \leq \tilde{N}_{\delta}$, where the constants $c_{i}, i=0,1,2$, are given by (2.14).

Proof. It is sufficient to show (ii) and (iii) by induction on $n$ with $0 \leq n \leq \tilde{N}_{\delta}$. Obviously, (ii) and (iii) are fulfilled with $n=0$. Now for any fixed $0 \leq l<\tilde{N}_{\delta}$, we assume that (ii) and (iii) hold true for all $0 \leq n \leq l$. We shall prove these assertions for $n=l+1$. To this end, we set for any $0 \leq m \leq l$

$$
z_{m}^{\delta}:=F\left(u_{m}^{\delta}\right)-y^{\dagger}-G_{m}^{\delta} e_{m}^{\delta} .
$$

Moreover, we see from (2.8) and the identity $I=\alpha(\alpha I+T)^{-1}+(\alpha I+T)^{-1} T$ that

$$
\begin{aligned}
e_{m+1}^{\delta}= & u_{m+1}^{\delta}-u^{\dagger} \\
= & e_{m}^{\delta}+\left(\alpha_{n} I+A_{m}^{\delta}\right)^{-1} G_{m}^{\delta *}\left(y^{\delta}-F\left(u_{m}^{\delta}\right)\right) \\
= & \alpha_{m}\left(\alpha_{m} I+A_{m}^{\delta}\right)^{-1} e_{m}^{\delta}+\left(\alpha_{m} I+A_{m}^{\delta}\right)^{-1} A_{m}^{\delta} e_{m}^{\delta} \\
& \quad+\left(\alpha_{m} I+A_{m}^{\delta}\right)^{-1} G_{m}^{\delta *}\left(y^{\delta}-F\left(u_{m}^{\delta}\right)\right) \\
= & \alpha_{m}\left(\alpha_{m} I+A_{m}^{\delta}\right)^{-1} e_{m}^{\delta}+\left(\alpha_{m} I+A_{m}^{\delta}\right)^{-1} G_{m}^{\delta *}\left(y^{\delta}-F\left(u_{m}^{\delta}\right)+G_{m}^{\delta} e_{m}^{\delta}\right) \\
= & \alpha_{m}\left(\alpha_{m} I+A_{m}^{\delta}\right)^{-1} e_{m}^{\delta}+\left(\alpha_{m} I+A_{m}^{\delta}\right)^{-1} G_{m}^{\delta *}\left(y^{\delta}-y^{\dagger}-z_{m}^{\delta}\right),
\end{aligned}
$$

which together with Lemma 2.3 gives

$$
\begin{aligned}
e_{m+1}^{\delta}=\alpha_{m} & \left(\alpha_{m} I+A\right)^{-1}\left[I+G_{\dagger}^{*} R_{\alpha_{m}}\left(u_{m}^{\delta}, u^{\dagger}\right)\right] e_{m}^{\delta} \\
& +\left(\alpha_{m} I+A\right)^{-1} G_{\dagger}^{*}\left[I+S_{\alpha_{m}}\left(u_{m}^{\delta}, u^{\dagger}\right)\right]\left(y^{\delta}-y^{\dagger}-z_{m}^{\delta}\right) .
\end{aligned}
$$

Consequently, it holds that

$$
e_{m+1}^{\delta}=\alpha_{m}\left(\alpha_{m} I+A\right)^{-1} e_{m}^{\delta}+\left(\alpha_{m} I+A\right)^{-1} G_{\uparrow}^{*} w_{m}^{\delta}
$$

with

$$
w_{m}^{\delta}:=\alpha_{m} R_{\alpha_{m}}\left(u_{m}^{\delta}, u^{\dagger}\right) e_{m}^{\delta}+\left[I+S_{\alpha_{m}}\left(u_{m}^{\delta}, u^{\dagger}\right)\right]\left(y^{\delta}-y^{\dagger}-z_{m}^{\delta}\right)
$$


The definition of $w_{m}^{\delta}$ and the estimates (2.13) imply that

$$
\left\|w_{m}^{\delta}\right\|_{Y} \leq \kappa(\rho) \alpha_{m}^{1 / 2}\left\|e_{m}^{\delta}\right\|_{U}+(1+3 \kappa(\rho))\left(\delta+\left\|z_{m}^{\delta}\right\|_{Y}\right)
$$

Furthermore, (GTCC) and (2.6) give

$$
\left\|z_{m}^{\delta}\right\|_{Y} \leq \frac{\eta(\rho)}{1-\eta_{0}}\left\|G_{\dagger} e_{m}^{\delta}\right\|_{Y}
$$

We thus have

$$
\left\|w_{m}^{\delta}\right\|_{Y} \leq \kappa(\rho) \alpha_{m}^{1 / 2}\left\|e_{m}^{\delta}\right\|_{U}+(1+3 \kappa(\rho))\left(\delta+\frac{\eta(\rho)}{1-\eta_{0}}\left\|G_{\dagger} e_{m}^{\delta}\right\|_{Y}\right)
$$

By telescoping (2.19), we obtain

$$
e_{l+1}^{\delta}=\prod_{m=0}^{l} \alpha_{m}\left(\alpha_{m} I+A\right)^{-1} e_{0}+\sum_{m=0}^{l} \alpha_{m}^{-1} \prod_{j=m}^{l} \alpha_{j}\left(\alpha_{j} I+A\right)^{-1} G_{\dagger}^{*} w_{m}^{\delta}
$$

and thus

$$
G_{\dagger} e_{l+1}^{\delta}=\prod_{m=0}^{l} \alpha_{m}\left(\alpha_{m} I+B\right)^{-1} G_{\dagger} e_{0}+\sum_{m=0}^{l} \alpha_{m}^{-1} \prod_{j=m}^{l} \alpha_{j}\left(\alpha_{j} I+B\right)^{-1} B w_{m}^{\delta}
$$

where we have used that $G_{\dagger}(\alpha I+A)^{-1}=(\alpha I+B)^{-1} G_{\dagger}$. Applying Lemmas A.3 and A.4 to (2.23) and using (2.22) yields

$$
\begin{aligned}
&\left\|e_{l+1}^{\delta}\right\|_{U} \leq\left\|e_{0}\right\|_{U}+ \frac{1}{2} \sum_{m=0}^{l} \alpha_{m}^{-1}\left(\sum_{j=m}^{l} \alpha_{j}^{-1}\right)^{-1 / 2}\left\|w_{m}^{\delta}\right\|_{Y} \\
& \leq\left\|e_{0}\right\|_{U}+\frac{1}{2} \sum_{m=0}^{l} \alpha_{m}^{-1}\left(\sum_{j=m}^{l} \alpha_{j}^{-1}\right)^{-1 / 2} \\
& \times\left[\kappa(\rho) \alpha_{m}^{1 / 2}\left\|e_{m}^{\delta}\right\|_{U}+(1+3 \kappa(\rho))\left(\delta+\frac{\eta(\rho)}{1-\eta_{0}}\left\|G_{\dagger} e_{m}^{\delta}\right\|_{Y}\right)\right] .
\end{aligned}
$$

We now use the induction hypothesis to deduce that

$$
\begin{aligned}
\left\|e_{l+1}^{\delta}\right\|_{U} \leq\left\|e_{0}\right\|_{U} & +\frac{1}{2} \sum_{m=0}^{l} \alpha_{m}^{-1}\left(\sum_{j=m}^{l} \alpha_{j}^{-1}\right)^{-1 / 2} \kappa(\rho) \alpha_{m}^{1 / 2}\left(2+c_{1} \gamma_{0}\right)\left\|e_{0}\right\|_{U} \\
+ & \frac{1}{2} \sum_{m=0}^{l} \alpha_{m}^{-1}\left(\sum_{j=m}^{l} \alpha_{j}^{-1}\right)^{-1 / 2} \\
& \times\left[(1+3 \kappa(\rho))\left(\delta+\frac{\eta(\rho)}{1-\eta_{0}}\left(c_{0}+2 c_{2} \gamma_{0}\right)\left\|e_{0}\right\|_{U} \alpha_{m}^{1 / 2}\right)\right]
\end{aligned}
$$


From the choice of $\tilde{N}_{\delta}$, it holds that $\delta \leq \gamma_{0}\left\|e_{0}\right\|_{U} \alpha_{m}^{1 / 2}$ for all $0 \leq m \leq l<\tilde{N}_{\delta}$. The above estimates and Lemma A.1 imply that

$$
\begin{aligned}
&\left\|e_{l+1}^{\delta}\right\|_{U} \leq\left\|e_{0}\right\|_{U}+\frac{1}{2} \sum_{m=0}^{l} \alpha_{m}^{-1 / 2}\left(\sum_{j=m}^{l} \alpha_{j}^{-1}\right)^{-1 / 2} \kappa(\rho)\left(2+c_{1} \gamma_{0}\right)\left\|e_{0}\right\|_{U} \\
&+ \frac{1}{2} \sum_{m=0}^{l} \alpha_{m}^{-1 / 2}\left(\sum_{j=m}^{l} \alpha_{j}^{-1}\right)^{-1 / 2} \\
& \times\left[(1+3 \kappa(\rho))\left(\gamma_{0}+\frac{\eta(\rho)}{1-\eta_{0}}\left(c_{0}+2 c_{2} \gamma_{0}\right)\right)\right]\left\|e_{0}\right\|_{U} \\
& \leq\left\|e_{0}\right\|_{U}+\frac{1}{2} c_{1}\left\|e_{0}\right\|_{U} \times\left[\kappa(\rho)\left(2+c_{1} \gamma_{0}\right)+(1+3 \kappa(\rho))\left(\gamma_{0}+\frac{\eta(\rho)}{1-\eta_{0}}\left(c_{0}+2 c_{2} \gamma_{0}\right)\right)\right] \\
&=\left\|e_{0}\right\|_{U}\left[1+\frac{c_{1}}{2} H_{1}(\rho)+\frac{c_{1}}{2} \gamma_{0}\left(1+H_{2}(\rho)\right)\right]
\end{aligned}
$$

with

$$
H_{1}(\rho):=2 \kappa(\rho)+(1+3 \kappa(\rho)) \frac{c_{0} \eta(\rho)}{1-\eta_{0}}, \quad H_{2}(\rho):=\left(c_{1}+3\right) \kappa(\rho)+\frac{2 c_{2} \eta(\rho)}{1-\eta_{0}}(1+3 \kappa(\rho)) .
$$

Combining this with the monotonic growth of $\kappa, \eta$ on $\left(0, \rho_{0}\right]$, the fact that $c_{0} /\left(2 c_{2}\right) \leq 2 / c_{1}$, and (2.17) yields

$$
\left\|e_{l+1}^{\delta}\right\|_{U} \leq\left\|e_{0}\right\|_{U}\left(2+c_{1} \gamma_{0}\right) .
$$

On the other hand, (2.24) along with Lemmas A.4 and A.3 gives

$$
\left\|G_{\dagger} e_{l+1}^{\delta}\right\|_{Y} \leq \frac{1}{2}\left(\sum_{j=0}^{l} \alpha_{j}^{-1}\right)^{-1 / 2}\left\|e_{0}\right\|_{U}+\sum_{m=0}^{l} \alpha_{m}^{-1}\left(\sum_{j=m}^{l} \alpha_{j}^{-1}\right)^{-1}\left\|w_{m}^{\delta}\right\|_{Y} .
$$

From this, Lemma A.1, and (2.22), the induction hypothesis and the choice of $\tilde{N}_{\delta}$ satisfying (2.16) lead to

$$
\begin{aligned}
& \left\|G_{\dagger} e_{l+1}^{\delta}\right\|_{Y} \\
& \leq \frac{1}{2} c_{0} \alpha_{l+1}^{1 / 2}\left\|e_{0}\right\|_{U}+\left\|e_{0}\right\|_{U} \sum_{m=0}^{l} \alpha_{m}^{-1 / 2}\left(\sum_{j=m}^{l} \alpha_{j}^{-1}\right)^{-1} \\
& \quad \times\left[\kappa(\rho)\left(2+c_{1} \gamma_{0}\right)+(1+3 \kappa(\rho))\left(\gamma_{0}+\frac{\eta(\rho)}{1-\eta_{0}}\left(c_{0}+2 c_{2} \gamma_{0}\right)\right)\right] \\
& \leq\left\|e_{0}\right\|_{U} \alpha_{l+1}^{1 / 2} \\
& \quad \times\left\{\frac{1}{2} c_{0}+c_{2}\left[\kappa(\rho)\left(2+c_{1} \gamma_{0}\right)+(1+3 \kappa(\rho))\left(\gamma_{0}+\frac{\eta(\rho)}{1-\eta_{0}}\left(c_{0}+2 c_{2} \gamma_{0}\right)\right)\right]\right\} \\
& \leq\left\|e_{0}\right\|_{U} \alpha_{l+1}^{1 / 2}\left[\frac{1}{2} c_{0}+c_{2} H_{1}(\rho)+c_{2} \gamma_{0}\left(1+H_{2}(\rho)\right)\right] .
\end{aligned}
$$


From this, the monotonic growth of $\kappa, \eta$ on $\left(0, \rho_{0}\right]$, and (2.17), we have that

$$
\left\|G_{\dagger} e_{l+1}^{\delta}\right\|_{Y} \leq\left(c_{0}+2 c_{2} \gamma_{0}\right)\left\|e_{0}\right\|_{U} \alpha_{l+1}^{1 / 2},
$$

which together with (2.25) implies that (ii) and (iii) are fulfilled with $n=l+1$.

By a similar argument for $\left\{u_{n}\right\}$ defined by (2.12), we obtain the following result:

Lemma 2.6 (cf. [8, Lem. 5]). Let $\left\{\alpha_{n}\right\}$ be defined by (2.9) and (2.10). Assume that Assumption (A1) and the first condition in (2.17) hold. Then, for any $u_{0} \in U$ such that $2\left\|e_{0}\right\|_{U}<\rho$ with $\rho \in\left(0, \rho_{1}\right]$, it holds that

(i) $u_{n} \in \bar{B}_{U}\left(u^{\dagger}, \rho\right)$;

(ii) $\left\|e_{n}\right\|_{U} \leq 2\left\|e_{0}\right\|_{U}<\rho$;

(iii) $\left\|G_{\dagger} e_{n}\right\|_{Y} \leq c_{0}\left\|e_{0}\right\|_{U} \alpha_{n}^{1 / 2}$

for all $n \geq 0$.

The next lemma is a crucial tool in our analysis to prove the well-posedness of the method as well as the asymptotic stability estimates.

LEMMA 2.7. Assume that all assumptions in Lemma 2.5 are satisfied. Then

$$
u_{n+1}^{\delta}-u_{n+1}=\alpha_{n}\left(\alpha_{n} I+A\right)^{-1}\left(u_{n}^{\delta}-u_{n}\right)+\sum_{i=1}^{5} s_{n}^{(i)} \quad \text { for all } 0 \leq n<\tilde{N}_{\delta},
$$

where

$$
s_{n}^{(i)}=\left(\alpha_{k} I+A\right)^{-1} G_{\dagger}^{*} \xi_{n}^{(i)}, \quad i=1, \ldots, 5,
$$

with some $\xi_{n}^{(i)} \in Y$ satisfying

$$
\begin{aligned}
\sum_{i=1}^{4}\left\|\xi_{n}^{(i)}\right\|_{Y} \leq L_{1}(\rho)\left(\alpha_{n}^{1 / 2}\left\|e_{n}\right\|_{U}+\alpha_{n}^{1 / 2}\left\|u_{n}^{\delta}-u_{n}\right\|_{U}\right. \\
\left.+\left\|G_{\dagger} e_{n}\right\|_{Y}+\left\|G_{\dagger}\left(u_{n}^{\delta}-u_{n}\right)\right\|_{Y}\right)
\end{aligned}
$$

and

$$
\xi_{n}^{(5)}=\left(y^{\delta}-y^{\dagger}\right)+\tilde{\xi}_{n}^{(5)} \quad \text { with } \quad\left\|\tilde{\xi}_{n}^{(5)}\right\|_{Y} \leq L_{1}(\rho) \delta
$$

for

$$
L_{1}(\rho):=\max \left\{3 \kappa(\rho),\left(1+3 \kappa_{0}\right)\left(2+\frac{3 \eta_{0}}{1-\eta_{0}}\right) \kappa(\rho), \frac{\left(1+3 \kappa_{0}\right)\left(1+\kappa_{0}\right)}{1-\eta_{0}} \eta(\rho)\right\} .
$$

Proof. For any $m \in \mathbb{N}$, we define

$$
z_{m}:=F\left(u_{m}\right)-y^{\dagger}-G_{m} e_{m} .
$$

We thus obtain from (2.8), (2.12), (2.18), and (2.31) that

$$
\begin{aligned}
& u_{n+1}^{\delta}-u_{n+1} \\
& \begin{aligned}
=u_{n}^{\delta}- & u_{n}+\left(\alpha_{n} I+A_{n}^{\delta}\right)^{-1} G_{n}^{\delta *}\left(y^{\delta}-F\left(u_{n}^{\delta}\right)\right)-\left(\alpha_{n} I+A_{n}\right)^{-1} G_{n}^{*}\left(y^{\dagger}-F\left(u_{n}\right)\right) \\
=u_{n}^{\delta}- & u_{n}+\left(\alpha_{n} I+A_{n}^{\delta}\right)^{-1} G_{n}^{\delta *}\left(y^{\delta}-y^{\dagger}-z_{n}^{\delta}-G_{n}^{\delta} e_{n}^{\delta}\right) \\
\quad & \quad+\left(\alpha_{n} I+A_{n}\right)^{-1} G_{n}^{*}\left(z_{n}+G_{n} e_{n}\right) \\
=u_{n}^{\delta}- & u_{n}+\left(\alpha_{n} I+A_{n}^{\delta}\right)^{-1} G_{n}^{\delta *}\left(y^{\delta}-y^{\dagger}\right)-\left(\alpha_{n} I+A_{n}^{\delta}\right)^{-1} G_{n}^{\delta *} z_{n}^{\delta} \\
\quad & \quad+\left(\alpha_{n} I+A_{n}\right)^{-1} G_{n}^{*} z_{n}+\left[\left(\alpha_{n} I+A_{n}\right)^{-1} A_{n} e_{n}-\left(\alpha_{n} I+A_{n}^{\delta}\right)^{-1} A_{n}^{\delta} e_{n}^{\delta}\right] .
\end{aligned}
\end{aligned}
$$


Furthermore, using the identity $(\alpha I+T)^{-1} T=I-\alpha(\alpha I+T)^{-1}$ gives

$$
\begin{aligned}
\left(\alpha_{n} I+A_{n}\right)^{-1} A_{n} e_{n}-\left(\alpha_{n} I+A_{n}^{\delta}\right)^{-1} A_{n}^{\delta} e_{n}^{\delta} & \\
=\left(\alpha_{n} I+A_{n}\right)^{-1} A_{n}\left(u_{n}-u_{n}^{\delta}\right)+ & {\left[\left(\alpha_{n} I+A_{n}\right)^{-1} A_{n}-\left(\alpha_{n} I+A_{n}^{\delta}\right)^{-1} A_{n}^{\delta}\right] e_{n}^{\delta} } \\
=\left(\alpha_{n} I+A\right)^{-1} A\left(u_{n}-u_{n}^{\delta}\right) & +\alpha_{n}\left[\left(\alpha_{n} I+A_{n}\right)^{-1}-\left(\alpha_{n} I+A\right)^{-1}\right]\left(u_{n}^{\delta}-u_{n}\right) \\
& +\alpha_{n}\left[\left(\alpha_{n} I+A_{n}^{\delta}\right)^{-1}-\left(\alpha_{n} I+A_{n}\right)^{-1}\right] e_{n}^{\delta}
\end{aligned}
$$

and thus

$$
\begin{aligned}
& \left(\alpha_{n} I+A_{n}\right)^{-1} A_{n} e_{n}-\left(\alpha_{n} I+A_{n}^{\delta}\right)^{-1} A_{n}^{\delta} e_{n}^{\delta} \\
& =\left(\alpha_{n} I+A\right)^{-1} A\left(u_{n}-u_{n}^{\delta}\right)+\alpha_{n}\left[\left(\alpha_{n} I+A_{n}^{\delta}\right)^{-1}-\left(\alpha_{n} I+A\right)^{-1}\right]\left(u_{n}^{\delta}-u_{n}\right) \\
& +\alpha_{n}\left[\left(\alpha_{n} I+A_{n}^{\delta}\right)^{-1}-\left(\alpha_{n} I+A_{n}\right)^{-1}\right] e_{n} .
\end{aligned}
$$

Defining

$$
\begin{aligned}
s_{n}^{(1)} & :=\alpha_{n}\left[\left(\alpha_{n} I+A_{n}^{\delta}\right)^{-1}-\left(\alpha_{n} I+A\right)^{-1}\right]\left(u_{n}^{\delta}-u_{n}\right), \\
s_{n}^{(2)} & :=\alpha_{n}\left[\left(\alpha_{n} I+A_{n}^{\delta}\right)^{-1}-\left(\alpha_{n} I+A_{n}\right)^{-1}\right] e_{n}, \\
s_{n}^{(3)} & :=\left[\left(\alpha_{n} I+A_{n}\right)^{-1} G_{n}^{*}-\left(\alpha_{n} I+A_{n}^{\delta}\right)^{-1} G_{n}^{\delta *}\right] z_{n}, \\
s_{n}^{(4)} & :=\left(\alpha_{n} I+A_{n}^{\delta}\right)^{-1} G_{n}^{\delta *}\left(z_{n}-z_{n}^{\delta}\right), \\
s_{n}^{(5)} & :=\left(\alpha_{n} I+A_{n}^{\delta}\right)^{-1} G_{n}^{\delta *}\left(y^{\delta}-y^{\dagger}\right)
\end{aligned}
$$

yields (2.26). We now verify (2.27), (2.28), and (2.29). To this end, we use Lemma 2.3 and obtain that

$$
s_{n}^{(1)}=\alpha_{n}\left(\alpha_{n} I+A\right)^{-1} G_{\dagger}^{*} R_{\alpha_{n}}\left(u_{n}^{\delta}, u^{\dagger}\right)\left(u_{n}^{\delta}-u_{n}\right),
$$

and so (2.27) holds for $i=1$ with

$$
\xi_{n}^{(1)}:=\alpha_{n} R_{\alpha_{n}}\left(u_{n}^{\delta}, u^{\dagger}\right)\left(u_{n}^{\delta}-u_{n}\right) .
$$

Note that $u_{n}^{\delta}, u_{n} \in \bar{B}\left(u^{\dagger}, \rho\right)$ according to Lemmas 2.5 and 2.6. We thus deduce from (2.13) that

$$
\left\|\xi_{n}^{(1)}\right\|_{Y} \leq \kappa(\rho) \alpha_{n}^{1 / 2}\left\|u_{n}^{\delta}-u_{n}\right\|_{U}
$$

Similarly, we obtain

$$
\left\{\begin{aligned}
\xi_{n}^{(2)} & :=\alpha_{n}\left[I+S_{\alpha_{n}}\left(u_{n}, u^{\dagger}\right)\right] R_{\alpha_{n}}\left(u_{n}^{\delta}, u_{n}\right) e_{n} \\
\xi_{n}^{(3)} & :=-\left[I+S_{\alpha_{n}}\left(u_{n}, u^{\dagger}\right)\right] S_{\alpha_{n}}\left(u_{n}^{\delta}, u_{n}\right) z_{n} \\
\xi_{n}^{(4)} & :=\left[I+S_{\alpha_{n}}\left(u_{n}^{\delta}, u^{\dagger}\right)\right]\left(z_{n}-z_{n}^{\delta}\right) \\
\xi_{n}^{(5)} & :=\left[I+S_{\alpha_{n}}\left(u_{n}^{\delta}, u^{\dagger}\right)\right]\left(y^{\delta}-y^{\dagger}\right)
\end{aligned}\right.
$$

and (2.27) then follows. Obviously, (2.29) is verified with $\tilde{\xi}_{n}^{(5)}:=S_{\alpha_{n}}\left(u_{n}^{\delta}, u^{\dagger}\right)\left(y^{\delta}-y^{\dagger}\right)$. It remains to prove the estimate (2.28). First, it is easy to see from (2.13) and the definition of $\xi_{n}^{(2)}$ in (2.33) that

$$
\left\|\xi_{n}^{(2)}\right\|_{Y} \leq(1+3 \kappa(\rho)) \kappa(\rho) \alpha_{n}^{1 / 2}\left\|e_{n}\right\|_{U}
$$


As a result of (GTCC), we have

$$
\left\|z_{n}\right\|_{Y} \leq \frac{\eta(\rho)}{1-\eta_{0}}\left\|G_{\dagger} e_{n}\right\|_{Y}
$$

which together with (2.13) and the definition of $\xi_{n}^{(3)}$ yields

$$
\left\|\xi_{n}^{(3)}\right\|_{Y} \leq \frac{3 \kappa(\rho) \eta(\rho)(1+3 \kappa(\rho))}{1-\eta_{0}}\left\|G_{\dagger} e_{n}\right\|_{Y}
$$

Furthermore, we can conclude from the definitions of $z_{n}^{\delta}$ and $z_{n}$, (GTCC), (2.3), and (2.4) that

$$
\begin{aligned}
\left\|z_{n}^{\delta}-z_{n}\right\|_{Y} & \leq\left\|F\left(u_{n}\right)-F\left(u_{n}^{\delta}\right)-G_{n}^{\delta}\left(u_{n}-u_{n}^{\delta}\right)\right\|_{Y}+\left\|\left(G_{n}-G_{n}^{\delta}\right) e_{n}\right\| Y \\
& \leq \frac{\eta(\rho)}{1-\eta_{0}}\left\|G_{n}\left(u_{n}-u_{n}^{\delta}\right)\right\|_{Y}+\left\|Q\left(u_{n}, u^{\dagger}\right)-Q\left(u_{n}^{\delta}, u^{\dagger}\right)\right\|_{\mathbb{L}(Y)}\left\|G_{\dagger} e_{n}\right\|_{Y} \\
& \leq \frac{\eta(\rho)}{1-\eta_{0}}\left\|Q\left(u_{n}, u^{\dagger}\right) G_{\dagger}\left(u_{n}-u_{n}^{\delta}\right)\right\|_{Y} \\
& +\left\|Q\left(u_{n}, u^{\dagger}\right)-Q\left(u_{n}^{\delta}, u^{\dagger}\right)\right\|_{\mathbb{L}(Y)}\left\|G_{\dagger} e_{n}\right\|_{Y} \\
& \leq \frac{\eta(\rho)}{1-\eta_{0}}(1+\kappa(\rho))\left\|G_{\dagger}\left(u_{n}-u_{n}^{\delta}\right)\right\|_{Y}+2 \kappa(\rho)\left\|G_{\dagger} e_{n}\right\|_{Y} .
\end{aligned}
$$

This, the definition of $\xi_{n}^{(4)}$, and (2.13) therefore imply that

$$
\left\|\xi_{n}^{(4)}\right\|_{Y} \leq(1+3 \kappa(\rho))\left[\frac{1+\kappa(\rho)}{1-\eta_{0}} \eta(\rho)\left\|G_{\dagger}\left(u_{n}-u_{n}^{\delta}\right)\right\|_{Y}+2 \kappa(\rho)\left\|G_{\dagger} e_{n}\right\|_{Y}\right] .
$$

From this, (2.32), (2.34), (2.35), and the monotonic growth of $\kappa$ on $\left(0, \rho_{0}\right]$, we obtain (2.28). $\square$

LEMMA 2.8. Let all assumptions of Lemma 2.7 be satisfied. Then it holds that

$$
\begin{aligned}
\left\|u_{l+1}^{\delta}-u_{l+1}\right\|_{U} \leq \frac{1}{2} c_{3}\left(1+L_{1}(\rho)\right) & \frac{\delta}{\sqrt{\alpha_{l+1}}} \\
& +\frac{L_{1}(\rho)}{2} \sum_{m=0}^{l} \alpha_{m}^{-1}\left(\sum_{j=m}^{l} \alpha_{j}^{-1}\right)^{-1 / 2} \sigma_{m}
\end{aligned}
$$

and

$$
\begin{aligned}
\left\|G_{\dagger}\left(u_{l+1}^{\delta}-u_{l+1}\right)-y^{\delta}+y^{\dagger}\right\|_{Y} \leq \delta & \left(1+c_{4} L_{1}(\rho)\right) \\
& +L_{1}(\rho) \sum_{m=0}^{l} \alpha_{m}^{-1}\left(\sum_{j=m}^{l} \alpha_{j}^{-1}\right)^{-1} \sigma_{m}
\end{aligned}
$$

for all $0 \leq l<\tilde{N}_{\delta}$ with $L_{1}(\rho)$ defined as (2.30) and

$$
\sigma_{m}:=\alpha_{m}^{1 / 2}\left\|e_{m}\right\|_{U}+\alpha_{m}^{1 / 2}\left\|u_{m}^{\delta}-u_{m}\right\|_{U}+\left\|G_{\dagger} e_{m}\right\|_{Y}+\left\|G_{\dagger}\left(u_{m}^{\delta}-u_{m}\right)\right\|_{Y} .
$$

Proof. Telescoping (2.26) and (2.27) gives

$$
u_{l+1}^{\delta}-u_{l+1}=\sum_{m=0}^{l} \alpha_{m}^{-1} \prod_{j=m}^{l} \alpha_{j}\left(\alpha_{j} I+A\right)^{-1} G_{\dagger}^{*} \sum_{i=1}^{5} \xi_{m}^{(i)}
$$

and thus 


$$
\begin{gathered}
G_{\dagger}\left(u_{l+1}^{\delta}-u_{l+1}\right)-\left(y^{\delta}-y^{\dagger}\right) \\
=\sum_{m=0}^{l} \alpha_{m}^{-1} \prod_{j=m}^{l} \alpha_{j}\left(\alpha_{j} I+B\right)^{-1} B\left[\sum_{i=1}^{4} \xi_{m}^{(i)}+\tilde{\xi}_{m}^{(5)}\right] \\
+\left[I-\sum_{m=0}^{l} \alpha_{m}^{-1} \prod_{j=m}^{l} \alpha_{j}\left(\alpha_{j} I+B\right)^{-1} B\right]\left(y^{\dagger}-y^{\delta}\right) \\
=\sum_{m=0}^{l} \alpha_{m}^{-1} \prod_{j=m}^{l} \alpha_{j}\left(\alpha_{j} I+B\right)^{-1} B\left[\sum_{i=1}^{4} \xi_{m}^{(i)}+\tilde{\xi}_{m}^{(5)}\right] \\
+\prod_{j=0}^{l} \alpha_{j}\left(\alpha_{j} I+B\right)^{-1}\left(y^{\dagger}-y^{\delta}\right),
\end{gathered}
$$

where we have used the identity

$$
I-\sum_{m=0}^{l} \alpha_{m}^{-1} \prod_{j=m}^{l} \alpha_{j}\left(\alpha_{j} I+B\right)^{-1} B=\prod_{j=0}^{l} \alpha_{j}\left(\alpha_{j} I+B\right)^{-1}
$$

to obtain the last equality. Applying Lemma A.4 to (2.38) and exploiting the estimates (2.28) as well as (2.29) yields

$$
\begin{aligned}
\left\|u_{l+1}^{\delta}-u_{l+1}\right\|_{U} & \leq \frac{1}{2} \sum_{m=0}^{l} \alpha_{m}^{-1}\left(\sum_{j=m}^{l} \alpha_{j}^{-1}\right)^{-1 / 2} \sum_{i=1}^{5}\left\|\xi_{m}^{(i)}\right\|_{Y} \\
\leq \frac{L_{1}(\rho)}{2} \sum_{m=0}^{l} \alpha_{m}^{-1}\left(\sum_{j=m}^{l} \alpha_{j}^{-1}\right)^{-1 / 2} & \times\left(\alpha_{m}^{1 / 2}\left\|e_{m}\right\|_{U}+\alpha_{m}^{1 / 2}\left\|u_{m}^{\delta}-u_{m}\right\|_{U}+\left\|G_{\dagger} e_{m}\right\|+\left\|G_{\dagger}\left(u_{m}^{\delta}-u_{m}\right)\right\|_{Y}\right) \\
+ & \frac{1}{2} \delta\left(1+L_{1}(\rho)\right) \sum_{m=0}^{l} \alpha_{m}^{-1}\left(\sum_{j=m}^{l} \alpha_{j}^{-1}\right)^{-1 / 2} .
\end{aligned}
$$

The estimate (2.36) then follows from the above estimate and Lemma A.1. Similarly, applying Lemma A.3 to (2.39), using Lemma A.1, and exploiting the estimates (2.28) as well as (2.29) yield

$$
\begin{aligned}
&\left\|G_{\dagger}\left(u_{l+1}^{\delta}-u_{l+1}\right)-y^{\delta}+y^{\dagger}\right\|_{Y} \\
& \leq \delta+\sum_{m=0}^{l} \alpha_{m}^{-1}\left(\sum_{j=m}^{l} \alpha_{j}^{-1}\right)^{-1}\left[\sum_{i=1}^{4}\left\|\xi_{m}^{(i)}\right\|_{Y}+\left\|\tilde{\xi}_{m}^{(5)}\right\|_{Y}\right] \\
& \leq L_{1}(\rho) \sum_{m=0}^{l} \alpha_{m}^{-1}\left(\sum_{j=m}^{l} \alpha_{j}^{-1}\right)^{-1} \\
& \times\left(\alpha_{m}^{1 / 2}\left\|e_{m}\right\|_{U}+\alpha_{m}^{1 / 2}\left\|u_{m}^{\delta}-u_{m}\right\|_{U}+\left\|G_{\dagger} e_{m}\right\|+\left\|G_{\dagger}\left(u_{m}^{\delta}-u_{m}\right)\right\|_{Y}\right) \\
&+\delta\left(1+c_{4} L_{1}(\rho)\right),
\end{aligned}
$$

which gives (2.37). 
COROLLARY 2.9. Under the assumptions in Lemma 2.8, it holds that

$$
\begin{aligned}
&\left\|G_{\dagger}\left(u_{l}^{\delta}-u_{l}\right)-y^{\delta}+y^{\dagger}\right\|_{Y} \\
& \leq \delta\left(1+c_{4} L_{1}(\rho)\right)+L_{1}(\rho) c_{2}\left[\left(6+3 c_{0}\right)+\gamma_{0}\left(c_{1}+2 c_{2}\right)\right]\left\|e_{0}\right\|_{U} \alpha_{l}^{1 / 2}
\end{aligned}
$$

and

$$
\left\|F\left(u_{l}^{\delta}\right)-F\left(u_{l}\right)-y^{\delta}+y^{\dagger}\right\|_{Y} \leq \delta\left(1+c_{4} L_{1}(\rho)\right)+\left(L_{2}(\rho)+\gamma_{0} L_{3}(\rho)\right)\left\|e_{0}\right\|_{U} \alpha_{l}^{1 / 2}
$$

for all $0 \leq l \leq \tilde{N}_{\delta}$ with

$$
\left\{\begin{array}{l}
L_{2}(\rho):=c_{2}\left(6+3 c_{0}\right) L_{1}(\rho)+2 c_{0}\left[\frac{\left(1+\kappa_{0}\right)}{1-\eta_{0}} \eta(\rho)+\kappa(\rho)\right], \\
L_{3}(\rho):=c_{2}\left(c_{1}+2 c_{2}\right) L_{1}(\rho)+2 c_{2}\left[\frac{\left(1+\kappa_{0}\right)}{1-\eta_{0}} \eta(\rho)+\kappa(\rho)\right] .
\end{array}\right.
$$

Proof. It suffices to prove (2.40) and (2.41) for all $1 \leq l \leq \tilde{N}_{\delta}$. According to (2.37), Lemmas 2.5 and 2.6, we have

$$
\begin{aligned}
& \left\|G_{\dagger}\left(u_{l}^{\delta}-u_{l}\right)-y^{\delta}+y^{\dagger}\right\|_{Y} \\
& \leq \delta\left(1+c_{4} L_{1}(\rho)\right)+L_{1}(\rho)\left[\left(6+3 c_{0}\right)+\gamma_{0}\left(c_{1}+2 c_{2}\right)\right]\left\|e_{0}\right\|_{U} \sum_{m=0}^{l-1} \alpha_{m}^{-1 / 2}\left(\sum_{j=m}^{l-1} \alpha_{j}^{-1}\right)^{-1},
\end{aligned}
$$

which along with Lemma A.1 gives (2.40). On the other hand, we can deduce from Assumption (A1) and (2.7) that

$$
\begin{aligned}
\| F\left(u_{l}^{\delta}\right)-F\left(u_{l}\right)- & y^{\delta}+y^{\dagger} \|_{Y} \\
\leq & \left\|G_{\dagger}\left(u_{l}^{\delta}-u_{l}\right)-y^{\delta}+y^{\dagger}\right\|_{Y} \\
& \quad+\left\|F\left(u_{l}^{\delta}\right)-F\left(u_{l}\right)-G_{l}\left(u_{l}^{\delta}-u_{l}\right)\right\|_{Y}+\left\|\left(G_{l}-G_{\dagger}\right)\left(u_{l}^{\delta}-u_{l}\right)\right\|_{Y} \\
\leq \| & G_{\dagger}\left(u_{l}^{\delta}-u_{l}\right)-y^{\delta}+y^{\dagger} \|_{Y} \\
\quad & \quad+\frac{\eta(\rho)}{1-\eta_{0}}\left\|G_{l}\left(u_{l}^{\delta}-u_{l}\right)\right\|_{Y}+\left\|\left(Q\left(u_{l}, u^{\dagger}\right)-I\right) G_{\dagger}\left(u_{l}^{\delta}-u_{l}\right)\right\|_{Y} \\
\leq \| & G_{\dagger}\left(u_{l}^{\delta}-u_{l}\right)-y^{\delta}+y^{\dagger} \|_{Y} \\
\quad & \quad \frac{\eta(\rho)}{1-\eta_{0}}\left\|Q\left(u_{l}, u^{\dagger}\right) G_{\dagger}\left(u_{l}^{\delta}-u_{l}\right)\right\|_{Y}+\kappa(\rho)\left\|G_{\dagger}\left(u_{l}^{\delta}-u_{l}\right)\right\|_{Y} \\
\leq \| & G_{\dagger}\left(u_{l}^{\delta}-u_{l}\right)-y^{\delta}+y^{\dagger} \|_{Y} \\
& \quad+\left[\frac{\left(1+\kappa_{0}\right)}{1-\eta_{0}} \eta(\rho)+\kappa(\rho)\right]\left\|G_{\dagger}\left(u_{l}^{\delta}-u_{l}\right)\right\|_{Y} .
\end{aligned}
$$

From this, (2.40), Lemmas 2.5 and 2.6, and the monotonic growth of $\kappa$, a simple computation verifies (2.41).

We finish this section by providing the logarithmic estimate of the stopping index $N_{\delta}$, where we again may have to further restrict the radius $\rho$ of the neighborhood of $u^{\dagger}$.

LEMMA 2.10. Let Assumption (A1) and (2.17) be fulfilled, and let $\left\{\alpha_{n}\right\}$ be defined by (2.9) and (2.10). Assume that $\tau>\tau_{0}>1, \gamma_{0} \geq \frac{2 c_{0}}{\left(1-\eta_{0}\right)\left(\tau-\tau_{0}\right)}$. Assume further that there exists a positive constant $\rho_{2} \leq \rho_{1}$, with $\rho_{1}$ given as in Lemma 2.5, such that

$$
c_{4} L_{1}\left(\rho_{2}\right)+L_{3}\left(\rho_{2}\right) \leq \tau_{0}-1 \quad \text { and } \quad L_{2}\left(\rho_{2}\right) \leq \frac{c_{0}}{1-\eta_{0}}
$$


with $L_{i}, 1 \leq i \leq 3$, defined as in (2.30) and (2.42). Let $\rho \in\left(0, \rho_{2}\right]$ and $u_{0} \in U$ be arbitrary such that $\left(2+c_{1} \gamma_{0}\right)\left\|e_{0}\right\|_{U}<\rho$. Then the modified Levenberg-Marquardt iteration (2.8)-(2.11) terminates after $N_{\delta}$ steps with

$$
N_{\delta}=O(1+|\log (\delta)|) .
$$

Proof. As a result of Lemma 2.4, it suffices to prove $N_{\delta} \leq \tilde{N}_{\delta}$. If $\tilde{N}_{\delta}=0$, then by definition we have $\alpha_{0}^{1 / 2}\left\|e_{0}\right\|_{U} \leq \frac{\delta}{\gamma_{0}}$. The estimate (2.6) thus gives

$$
\begin{aligned}
\left\|F\left(u_{0}\right)-y^{\delta}\right\|_{Y} & \leq\left\|y^{\dagger}-y^{\delta}\right\|_{Y}+\left\|F\left(u_{0}\right)-y^{\dagger}\right\|_{Y} \leq \delta+\frac{1}{1-\eta_{0}}\left\|G_{\dagger} e_{0}\right\|_{Y} \\
& \leq \delta+\frac{1}{1-\eta_{0}}\left\|e_{0}\right\|_{U} \alpha_{0}^{1 / 2} \leq \delta\left(1+\frac{1}{\left(1-\eta_{0}\right) \gamma_{0}}\right)<\tau \delta .
\end{aligned}
$$

In the following we shall assume $\tilde{N}_{\delta}>0$. We deduce from (2.41) for $l=\tilde{N}_{\delta}$ that

$$
\left\|F\left(u_{\tilde{N}_{\delta}}^{\delta}\right)-F\left(u_{\tilde{N}_{\delta}}\right)-y^{\delta}+y^{\dagger}\right\|_{Y} \leq \delta\left(1+c_{4} L_{1}(\rho)\right)+\left(L_{2}(\rho)+\gamma_{0} L_{3}(\rho)\right)\left\|e_{0}\right\|_{U} \alpha_{\tilde{N}_{\delta}}^{1 / 2} .
$$

Using (2.6), Lemma 2.6, and noting that $\alpha_{\tilde{N}_{\delta}}^{1 / 2}\left\|e_{0}\right\|_{U} \leq \frac{\delta}{\gamma_{0}}$, we derive

$$
\begin{aligned}
\| y^{\delta}- & F\left(u_{\tilde{N}_{\delta}}^{\delta}\right)\left\|_{Y} \leq\right\| F\left(u_{\tilde{N}_{\delta}}^{\delta}\right)-F\left(u_{\tilde{N}_{\delta}}\right)-y^{\delta}+y^{\dagger}\left\|_{Y}+\right\| F\left(u_{\tilde{N}_{\delta}}\right)-y^{\dagger} \|_{Y} \\
& \leq\left\|F\left(u_{\tilde{N}_{\delta}}^{\delta}\right)-F\left(u_{\tilde{N}_{\delta}}\right)-y^{\delta}+y^{\dagger}\right\|_{Y}+\frac{1}{1-\eta_{0}}\left\|G_{\dagger} e_{\tilde{N}_{\delta}}\right\|_{Y} \\
& \leq \delta\left(1+c_{4} L_{1}(\rho)\right)+\left(L_{2}(\rho)+\gamma_{0} L_{3}(\rho)\right)\left\|e_{0}\right\|_{U} \alpha_{\tilde{N}_{\delta}}^{1 / 2}+\frac{c_{0}}{1-\eta_{0}}\left\|e_{0}\right\|_{U} \alpha_{\tilde{N}_{\delta}}^{1 / 2} \\
& \leq \delta\left[1+\left(c_{4} L_{1}(\rho)+L_{3}(\rho)\right)+\frac{1}{\gamma_{0}}\left(\frac{c_{0}}{1-\eta_{0}}+L_{2}(\rho)\right)\right] .
\end{aligned}
$$

Combining this with (2.43), the definitions of $L_{i}, 1 \leq i \leq 3$, and the monotonic growth of $\kappa, \eta$, we obtain

$$
\left\|y^{\delta}-F\left(u_{\tilde{N}_{\delta}}^{\delta}\right)\right\|_{Y} \leq \delta\left(\tau_{0}+\frac{1}{\gamma_{0}} \frac{2 c_{0}}{1-\eta_{0}}\right) \leq \delta \tau .
$$

From this and the definition of $N_{\delta}$, we have $N_{\delta} \leq \tilde{N}_{\delta}$.

2.2. Convergence in the noise-free setting. In this section we will show convergence of the sequence $\left\{u_{n}\right\}$ defined via (2.12) provided that $e_{0} \in \mathcal{N}\left(G_{\dagger}\right)^{\perp}$ and that the parameter $\eta(\rho)$ and $\kappa(\rho)$ are small enough if the radius $\rho$ can be chosen accordingly.

We first derive some estimates for $e_{n}$ and $G_{\dagger} e_{n}$ under the generalized source condition

$$
e_{0}=A^{\nu} w
$$

for some $\nu \in\left(0, \frac{1}{2}\right)$ and some $w \in U$, where $A=G_{\dagger}^{*} G_{\dagger}$. Again, we may have to restrict $\rho$ further.

LEMMA 2.11. Let all assumptions in Lemma 2.6 hold. Assume further that there exists a constant $\bar{\rho}_{1} \in\left(0, \rho_{1}\right]$, with $\rho_{1}$ given as in Lemma 2.5 , satisfying

$$
\kappa\left(\bar{\rho}_{1}\right)+c_{0} \frac{1+3 \kappa_{0}}{1-\eta_{0}} \eta\left(\bar{\rho}_{1}\right) \leq \frac{c_{0}}{2 K_{1}(r, \nu)}
$$


for some constant $\nu \in\left(0, \frac{1}{2}\right)$. Let $\rho \in\left(0, \bar{\rho}_{1}\right]$ be arbitrary, and let $u_{0} \in U$ satisfy $2\left\|e_{0}\right\|_{U}<\rho$ and $e_{0}=A^{\nu} w$ for some $w \in U$. Then

$$
\left\|e_{n}\right\|_{U} \leq 2 c_{0} \alpha_{n}^{\nu}\|w\|_{U} \quad \text { and } \quad\left\|G_{\dagger} e_{n}\right\|_{Y} \leq 2 c_{0}^{2} \alpha_{n}^{\nu+1 / 2}\|w\|_{U}
$$

for all $n \geq 0$.

Proof. We shall prove the lemma by induction on $n$. Obviously, (2.46) is valid for $n=0$. We now assume that (2.46) holds for all $0 \leq n \leq l$ and prove that this is also true for $n=l+1$. An argument similar to the one used to obtain (2.23) and (2.24) yields

$$
e_{l+1}=\prod_{m=0}^{l} \alpha_{m}\left(\alpha_{m} I+A\right)^{-1} e_{0}+\sum_{m=0}^{l} \alpha_{m}^{-1} \prod_{j=m}^{l} \alpha_{j}\left(\alpha_{j} I+A\right)^{-1} G_{\dagger}^{*} w_{m}
$$

and

$$
G_{\dagger} e_{l+1}=\prod_{m=0}^{l} \alpha_{m}\left(\alpha_{m} I+B\right)^{-1} G_{\dagger} e_{0}+\sum_{m=0}^{l} \alpha_{m}^{-1} \prod_{j=m}^{l} \alpha_{j}\left(\alpha_{j} I+B\right)^{-1} B w_{m},
$$

where, analogous to (2.20),

$$
w_{m}:=\alpha_{m} R_{\alpha_{m}}\left(u_{m}, u^{\dagger}\right) e_{m}-\left[I+S_{\alpha_{m}}\left(u_{m}, u^{\dagger}\right)\right] z_{m}
$$

for all $0 \leq m \leq l$ with $z_{m}$ defined via (2.31). Similarly to (2.22), we have

$$
\left\|w_{m}\right\|_{Y} \leq \kappa(\rho) \alpha_{m}^{1 / 2}\left\|e_{m}\right\|_{U}+(1+3 \kappa(\rho)) \frac{\eta(\rho)}{1-\eta_{0}}\left\|G_{\dagger} e_{m}\right\|_{Y} .
$$

This and the induction hypothesis yield

$$
\left\|w_{m}\right\|_{Y} \leq Q_{1}(\rho) \alpha_{m}^{\nu+1 / 2}\|w\|_{U}
$$

for all $0 \leq m \leq l$ with

$$
Q_{1}(\rho):=2 c_{0} \kappa(\rho)+2 c_{0}^{2} \frac{1+3 \kappa(\rho)}{1-\eta_{0}} \eta(\rho) .
$$

Inserting $e_{0}=A^{\nu} w$ into (2.47) and then applying Lemmas A.3 and A.4, we deduce that

$$
\begin{aligned}
\left\|e_{l+1}\right\|_{U} & \leq\|w\|_{U}\left(\sum_{j=m}^{l} \alpha_{j}^{-1}\right)^{-\nu}+\frac{1}{2} \sum_{m=0}^{l} \alpha_{m}^{-1}\left(\sum_{j=m}^{l} \alpha_{j}^{-1}\right)^{-1 / 2}\left\|w_{m}\right\|_{Y} \\
& \leq c_{0}^{2 \nu} \alpha_{l+1}^{\nu}\|w\|_{U}+\frac{1}{2} Q_{1}(\rho)\|w\|_{U} \sum_{m=0}^{l} \alpha_{m}^{\nu-1 / 2}\left(\sum_{j=m}^{l} \alpha_{j}^{-1}\right)^{-1 / 2} \\
& \leq c_{0} \alpha_{l+1}^{\nu}\|w\|_{U}+\frac{1}{2} Q_{1}(\rho) K_{0}(r, \nu) \alpha_{l+1}^{\nu}\|w\|_{U}
\end{aligned}
$$

where we used (2.49) and Lemma A.1 to obtain the second inequality and Lemma A.2 to obtain the last inequality. By virtue of (2.45), the fact that $c_{0} /\left(2 K_{1}(r, \nu)\right) \leq 1 / K_{0}(r, \nu)$, and the monotonic growth of $\kappa$ and $\eta$, it holds that

$$
\left\|e_{l+1}\right\|_{U} \leq 2 c_{0} \alpha_{l+1}^{\nu}\|w\|_{U} .
$$


Moreover, by inserting $e_{0}=A^{\nu} w$ into (2.48), Corollary A.5, Lemma A.3, and (2.49) reveal that

$$
\begin{aligned}
\left\|G_{\dagger} e_{l+1}\right\|_{Y} & \leq\|w\|_{U}\left(\sum_{j=0}^{l} \alpha_{j}^{-1}\right)^{-\nu-1 / 2}+\sum_{m=0}^{l} \alpha_{m}^{-1}\left(\sum_{j=m}^{l} \alpha_{j}^{-1}\right)^{-1}\left\|w_{m}\right\|_{Y} \\
& \leq c_{0}^{2 \nu+1} \alpha_{l+1}^{\nu+1 / 2}\|w\|_{U}+Q_{1}(\rho)\|w\|_{U} \sum_{m=0}^{l} \alpha_{m}^{\nu-1 / 2}\left(\sum_{j=m}^{l} \alpha_{j}^{-1}\right)^{-1} \\
& \leq c_{0}^{2} \alpha_{l+1}^{\nu+1 / 2}\|w\|_{U}+Q_{1}(\rho) K_{1}(r, \nu) \alpha_{l+1}^{\nu+1 / 2}\|w\|_{U}
\end{aligned}
$$

Here the second estimate is derived using Lemma A.1 while the last estimate is obtained using Lemma A.2. Then

$$
\left\|G_{\dagger} e_{l+1}\right\|_{Y} \leq 2 c_{0}^{2} \alpha_{l+1}^{\nu+1 / 2}\|w\|_{U} .
$$

From (2.50) and (2.51), we conclude that (2.46) is fulfilled with $n=l+1$.

We now take $\hat{u}_{0} \in U$ to be a perturbation of $u_{0} \in U$ and denote by $\left\{\hat{u}_{n}\right\}$ the iterates given by (2.12) with $u_{0}$ replaced by $\hat{u}_{0}$, that is,

$$
\hat{u}_{n+1}=\hat{u}_{n}+\left(\alpha_{n} I+G_{\hat{u}_{n}}^{*} G_{\hat{u}_{n}}\right)^{-1} G_{\hat{u}_{n}}^{*}\left(y^{\dagger}-F\left(\hat{u}_{n}\right)\right), \quad n=0,1, \ldots
$$

For the ease of exposition, from now on we use the notations

$$
\begin{array}{ll}
\hat{e}_{n}:=\hat{u}_{n}-u^{\dagger}, & \hat{G}_{n}:=G_{\hat{u}_{n}}, \quad \hat{A}_{n}:=\hat{G}_{n}^{*} \hat{G}_{n}, \quad \hat{B}_{n}:=\hat{G}_{n} \hat{G}_{n}^{*}, \\
\hat{z}_{n}:=F\left(\hat{u}_{n}\right)-y^{\dagger}-\hat{G}_{n} \hat{e}_{n} . &
\end{array}
$$

The next lemma is analogous to Lemma 2.7 .

LEMMA 2.12. Let all assumptions in Lemma 2.6 be fulfilled, and let $\bar{\rho}_{1} \in\left(0, \rho_{1}\right]$ and $\nu \in\left(0, \frac{1}{2}\right)$ satisfy (2.45). Assume that $\rho \in\left(0, \bar{\rho}_{1}\right]$ and that $u_{0}, \hat{u}_{0} \in U$ satisfy $\min \left\{2\left\|e_{0}\right\|_{U}, 2\left\|\hat{e}_{0}\right\|_{U}\right\}<\rho$ and $\hat{e}_{0}=A^{\nu} w$ for some $w \in U$. Then for all $k \geq 0$, it holds that

$$
u_{k+1}-\hat{u}_{k+1}=\alpha_{k}\left(\alpha_{k} I+A\right)^{-1}\left(u_{k}-\hat{u}_{k}\right)+\sum_{i=1}^{4} t_{k}^{(i)}
$$

where

$$
t_{k}^{(i)}=\left(\alpha_{k} I+A\right)^{-1} G_{\dagger}^{*} h_{k}^{(i)}, \quad i=1,2,3,4,
$$

with some $h_{k}^{(i)} \in Y$ satisfying

$$
\sum_{i=1}^{4}\left\|h_{k}^{(i)}\right\|_{Y} \leq C(\rho)\left[\|w\|_{U} \alpha_{k}^{\nu+1 / 2}+\left\|u_{k}-\hat{u}_{k}\right\|_{U} \alpha_{k}^{1 / 2}+\left\|G_{\dagger}\left(u_{k}-\hat{u}_{k}\right)\right\|_{Y}\right]
$$

for

(2.56) $C(\rho):=\left(1+3 \kappa_{0}\right) \max \left\{2 c_{0}\left(\frac{3 c_{0} \eta_{0}}{1-\eta_{0}}+1+2 c_{0}\left(1+\kappa_{0}\right)\right) \kappa(\rho), \frac{\left(1+\kappa_{0}\right)}{1-\eta_{0}} \eta(\rho)\right\}$. 
Proof. Analogous to (2.26), we see from (2.12), (2.52), (2.31), and the definition of $\hat{z}_{k}$ that (2.53) is satisfied with

$$
\begin{aligned}
t_{k}^{(1)} & :=\alpha_{k}\left[\left(\alpha_{k} I+A_{k}\right)^{-1}-\left(\alpha_{k} I+A\right)^{-1}\right]\left(u_{k}-\hat{u}_{k}\right), \\
t_{k}^{(2)} & :=\alpha_{k}\left[\left(\alpha_{k} I+A_{k}\right)^{-1}-\left(\alpha_{k} I+\hat{A}_{k}\right)^{-1}\right] \hat{e}_{k}, \\
t_{k}^{(3)} & :=\left[\left(\alpha_{k} I+\hat{A}_{k}\right)^{-1} \hat{G}_{k}^{*}-\left(\alpha_{k} I+A_{k}\right)^{-1} G_{k}^{*}\right] \hat{z}_{k}, \\
t_{k}^{(4)} & :=\left(\alpha_{k} I+A_{k}\right)^{-1} G_{k}^{*}\left(\hat{z}_{k}-z_{k}\right) .
\end{aligned}
$$

We now prove (2.54) and (2.55). To verify these relations, we use Lemma 2.3 to obtain

$$
t_{k}^{(1)}=\alpha_{k}\left(\alpha_{k} I+A\right)^{-1} G_{\dagger}^{*} R_{\alpha_{k}}\left(u_{k}, u^{\dagger}\right)\left(u_{k}-\hat{u}_{k}\right)
$$

and thus $h_{k}^{(1)}:=\alpha_{k} R_{\alpha_{k}}\left(u_{k}, u^{\dagger}\right)\left(u_{k}-\hat{u}_{k}\right)$ verifies (2.54) for $i=1$. The estimate (2.13) then implies that

$$
\left\|h_{k}^{(1)}\right\|_{Y} \leq \kappa(\rho)\left\|u_{k}-\hat{u}_{k}\right\|_{U} \alpha_{k}^{1 / 2}
$$

Furthermore, we have

$$
\begin{aligned}
t_{k}^{(2)} & =\alpha_{k}\left(\alpha_{k} I+\hat{A}_{k}\right)^{-1} \hat{G}_{k}^{*} R_{\alpha_{k}}\left(u_{k}, \hat{u}_{k}\right) \hat{e}_{k} \\
& =\alpha_{k}\left(\alpha_{k} I+A\right)^{-1} G_{\dagger}^{*}\left[I+S_{\alpha_{k}}\left(\hat{u}_{k}, u^{\dagger}\right)\right] R_{\alpha_{k}}\left(u_{k}, \hat{u}_{k}\right) \hat{e}_{k},
\end{aligned}
$$

and so (2.54) is valid for $i=2$ with

$$
h_{k}^{(2)}:=\alpha_{k}\left[I+S_{\alpha_{k}}\left(\hat{u}_{k}, u^{\dagger}\right)\right] R_{\alpha_{k}}\left(u_{k}, \hat{u}_{k}\right) \hat{e}_{k} .
$$

This and (2.13) yield

$$
\left\|h_{k}^{(2)}\right\|_{Y} \leq(1+3 \kappa(\rho)) \kappa(\rho) \alpha_{k}^{1 / 2}\left\|\hat{e}_{k}\right\|_{U} \leq 2 c_{0}\left(1+3 \kappa_{0}\right) \kappa(\rho)\|w\|_{U} \alpha_{k}^{\nu+1 / 2},
$$

where we have used Lemma 2.11 and the monotonic growth of $\kappa$ to obtain the last estimate. Noting that $u_{k}, \hat{u}_{k} \in \bar{B}\left(u^{\dagger}, \rho\right)$, according to Lemma 2.6, we have

$$
t_{k}^{(3)}=\left(\alpha_{k} I+A\right)^{-1} G_{\dagger}^{*}\left[I+S_{\alpha_{k}}\left(u_{k}, u^{\dagger}\right)\right] S_{\alpha_{k}}\left(\hat{u}_{k}, u_{k}\right) \hat{z}_{k}
$$

and therefore $h_{k}^{(3)}:=\left[I+S_{\alpha_{k}}\left(u_{k}, u^{\dagger}\right)\right] S_{\alpha_{k}}\left(\hat{u}_{k}, u_{k}\right) \hat{z}_{k}$. The estimate (2.13) then yields

$$
\left\|h_{k}^{(3)}\right\|_{Y} \leq 3\left(1+3 \kappa_{0}\right) \kappa(\rho)\left\|\hat{z}_{k}\right\|_{Y} .
$$

On the other hand, as a result of (GTCC) and Lemma 2.11, we have

$$
\left\|\hat{z}_{k}\right\|_{Y} \leq \frac{\eta(\rho)}{1-\eta_{0}}\left\|G_{\dagger} \hat{e}_{k}\right\|_{Y} \leq 2 c_{0}^{2} \frac{\eta(\rho)}{1-\eta_{0}}\|w\|_{U} \alpha_{k}^{\nu+1 / 2}
$$

The two estimates above show that $h_{k}^{(3)}$ satisfies

$$
\left\|h_{k}^{(3)}\right\|_{Y} \leq 6 c_{0}^{2}\left(1+3 \kappa_{0}\right) \kappa(\rho) \frac{\eta(\rho)}{1-\eta_{0}}\|w\|_{U} \alpha_{k}^{\nu+1 / 2} .
$$


Finally,

$$
t_{k}^{(4)}=\left(\alpha_{k} I+A\right)^{-1} G_{\dagger}^{*}\left[I+S_{\alpha_{k}}\left(u_{k}, u^{\dagger}\right)\right]\left(\hat{z}_{k}-z_{k}\right)=\left(\alpha_{k} I+A\right)^{-1} G_{\dagger}^{*} h_{k}^{(4)}
$$

with $h_{k}^{(4)}:=\left[I+S_{\alpha_{k}}\left(u_{k}, u^{\dagger}\right)\right]\left(\hat{z}_{k}-z_{k}\right)$. From this and (2.13), we obtain

$$
\left\|h_{k}^{(4)}\right\|_{Y} \leq\left(1+3 \kappa_{0}\right)\left\|\hat{z}_{k}-z_{k}\right\|_{Y} .
$$

From the definitions of $z_{k}$ and $\hat{z}_{k}$, it follows that

$$
\begin{aligned}
\left\|\hat{z}_{k}-z_{k}\right\|_{Y} & =\left\|F\left(\hat{u}_{k}\right)-F\left(u_{k}\right)-G_{k}\left(\hat{u}_{k}-u_{k}\right)+\left(G_{k}-\hat{G}_{k}\right) \hat{e}_{k}\right\|_{Y} \\
& \leq\left\|F\left(\hat{u}_{k}\right)-F\left(u_{k}\right)-G_{k}\left(\hat{u}_{k}-u_{k}\right)\right\|_{Y}+\left\|\left(G_{k}-\hat{G}_{k}\right) \hat{e}_{k}\right\|_{Y} \\
& \leq \frac{\eta(\rho)}{1-\eta_{0}}\left\|G_{k}\left(\hat{u}_{k}-u_{k}\right)\right\|_{Y}+\left\|\left(G_{k}-\hat{G}_{k}\right) \hat{e}_{k}\right\|_{Y} .
\end{aligned}
$$

Here we used (2.5). Combining this with (2.3), (2.4), and (2.7), we obtain

$$
\begin{aligned}
\left\|\hat{z}_{k}-z_{k}\right\|_{Y} & \leq \frac{\eta(\rho)}{1-\eta_{0}}\left(1+\kappa_{0}\right)\left\|G_{\dagger}\left(\hat{u}_{k}-u_{k}\right)\right\|_{Y}+2 \kappa(\rho)\left(1+\kappa_{0}\right)\left\|G_{\dagger} \hat{e}_{k}\right\|_{Y} \\
& \leq\left(1+\kappa_{0}\right)\left[\frac{\eta(\rho)}{1-\eta_{0}}\left\|G_{\dagger}\left(\hat{u}_{k}-u_{k}\right)\right\|_{Y}+4 c_{0}^{2} \kappa(\rho)\|w\|_{U} \alpha_{k}^{\nu+1 / 2}\right],
\end{aligned}
$$

where we have used (2.46) to get the last inequality. This together with (2.60) shows that

$$
\left\|h_{k}^{(4)}\right\|_{Y} \leq\left(1+3 \kappa_{0}\right)\left(1+\kappa_{0}\right)\left[\frac{\eta(\rho)}{1-\eta_{0}}\left\|G_{\dagger}\left(\hat{u}_{k}-u_{k}\right)\right\|_{Y}+4 c_{0}^{2} \kappa(\rho)\|w\|_{U} \alpha_{k}^{\nu+1 / 2}\right] .
$$

Summing up from (2.57)-(2.59) to (2.61) yields (2.55).

LEMMA 2.13. Let all assumptions in Lemma 2.6 be fulfilled, and let $\bar{\rho}_{1}, \nu$ be defined as in Lemma 2.11. Assume that there exists a constant $\bar{\rho}_{2} \in\left(0, \bar{\rho}_{1}\right]$ satisfying

$$
C\left(\bar{\rho}_{2}\right) \leq \min \left\{\frac{c_{0}}{2 c_{2}\left(2+c_{0}\right)}, \frac{1}{K_{0}(r, \nu)+2 K_{1}(r, \nu)}\right\},
$$

where $C(\rho)$ is defined by (2.56). Let $\rho \in\left(0, \bar{\rho}_{2}\right]$ be arbitrary. Assume in addition that $u_{0}, \hat{u}_{0} \in U$ are such that $\min \left\{2\|\hat{e}\|_{U}, 2\left\|\hat{e}_{0}\right\|_{U}\right\}<\rho$ and $\hat{e}_{0}=A^{\nu} w$ for some $w \in U$. Then it holds that

$$
\left\|u_{n}-\hat{u}_{n}\right\|_{U} \leq 2\left\|u_{0}-\hat{u}_{0}\right\|_{U}+\pi_{1}(\rho)\|w\|_{U} \alpha_{n}^{\nu}
$$

and

$$
\left\|G_{\dagger}\left(u_{n}-\hat{u}_{n}\right)\right\|_{U} \leq c_{0} \alpha_{n}^{1 / 2}\left\|u_{0}-\hat{u}_{0}\right\|_{U}+\pi_{2}(\rho)\|w\|_{U} \alpha_{n}^{\nu+1 / 2}
$$

for all $n \geq 0$. Here

$$
\pi_{1}(\rho):=C(\rho) K_{0}(r, \nu), \quad \pi_{2}(\rho):=2 C(\rho) K_{1}(r, \nu) .
$$

Proof. We show (2.63) and (2.64) by induction on $n$. Easily, these estimates hold for $n=0$. Assume that (2.63) and (2.64) are satisfied for all $0 \leq n \leq l$. We shall prove that these estimates also hold for $n=l+1$. To that purpose, we apply Lemma 2.12 to obtain

$$
\begin{aligned}
u_{l+1}-\hat{u}_{l+1}=\prod_{m=0}^{l} \alpha_{m}\left(\alpha_{m} I+A\right)^{-1}\left(u_{0}-\hat{u}_{0}\right) & \\
& \quad+\sum_{m=0}^{l} \alpha_{m}^{-1} \prod_{j=m}^{l} \alpha_{j}\left(\alpha_{j} I+A\right)^{-1} G_{\dagger}^{*} \sum_{i=1}^{4} h_{m}^{(i)}
\end{aligned}
$$


and

$$
\begin{aligned}
G_{\dagger}\left(u_{l+1}-\hat{u}_{l+1}\right)=\prod_{m=0}^{l} \alpha_{m}\left(\alpha_{m} I+B\right)^{-1} G_{\dagger}\left(u_{0}-\hat{u}_{0}\right) \\
\quad+\sum_{m=0}^{l} \alpha_{m}^{-1} \prod_{j=m}^{l} \alpha_{j}\left(\alpha_{j} I+B\right)^{-1} B \sum_{i=1}^{4} h_{m}^{(i)} .
\end{aligned}
$$

Applying Lemma A.3 and Lemma A.4 to (2.65) and using Lemma 2.12, we obtain

$$
\begin{aligned}
\left\|u_{l+1}-\hat{u}_{l+1}\right\|_{U} \leq\left\|u_{0}-\hat{u}_{0}\right\|_{U}+\frac{1}{2} \sum_{m=0}^{l} \alpha_{m}^{-1}\left(\sum_{j=m}^{l} \alpha_{j}^{-1}\right)^{-1 / 2} \sum_{i=1}^{4}\left\|h_{m}^{(i)}\right\|_{Y} \\
\leq\left\|u_{0}-\hat{u}_{0}\right\|_{U}+\frac{1}{2} C(\rho) \sum_{m=0}^{l} \alpha_{m}^{-1}\left(\sum_{j=m}^{l} \alpha_{j}^{-1}\right)^{-1 / 2} \\
\times\left[\alpha_{m}^{\nu+1 / 2}\|w\|_{U}+\alpha_{m}^{1 / 2}\left\|u_{m}-\hat{u}_{m}\right\|_{U}+\left\|G_{\dagger}\left(u_{m}-\hat{u}_{m}\right)\right\|_{Y}\right],
\end{aligned}
$$

which together with the induction hypothesis as well as Lemmas A.1 and A.2 shows that

$$
\begin{aligned}
\left\|u_{l+1}-\hat{u}_{l+1}\right\|_{U} & \\
\leq\left\|u_{0}-\hat{u}_{0}\right\|_{U} & +\frac{1}{2} C(\rho)\left(2+c_{0}\right)\left\|u_{0}-\hat{u}_{0}\right\|_{U} \sum_{m=0}^{l} \alpha_{m}^{-1 / 2}\left(\sum_{j=m}^{l} \alpha_{j}^{-1}\right)^{-1 / 2} \\
& +\frac{1}{2} C(\rho)\left(1+\pi_{1}(\rho)+\pi_{2}(\rho)\right)\|w\|_{U} \sum_{m=0}^{l} \alpha_{m}^{\nu-1 / 2}\left(\sum_{j=m}^{l} \alpha_{j}^{-1}\right)^{-1 / 2} \\
\leq\left\|u_{0}-\hat{u}_{0}\right\|_{U} & {\left[1+\frac{1}{2} C(\rho)\left(2+c_{0}\right) c_{1}\right] } \\
& +\frac{1}{2} C(\rho) K_{0}(r, \nu)\left(1+\pi_{1}(\rho)+\pi_{2}(\rho)\right)\|w\|_{U} \alpha_{l+1}^{\nu} .
\end{aligned}
$$

Thanks to (2.62), the definition of $C(\rho)$, the fact that $c_{0} /\left(2 c_{2}\right) \leq 2 / c_{1}$, and the monotonic growth of $\kappa, \eta$, we obtain

$$
\left\|u_{l+1}-\hat{u}_{l+1}\right\|_{U} \leq 2\left\|u_{0}-\hat{u}_{0}\right\|_{U}+C(\rho) K_{0}(r, \nu)\|w\|_{U} \alpha_{l+1}^{\nu} .
$$

This verifies (2.63) for $n=l+1$. It remains to prove (2.64) for $n=l+1$. To this end, using similar argument as above, we obtain from (2.66), (2.55), Lemmas A.4 and A.3 that

$$
\begin{aligned}
&\left\|G_{\dagger}\left(u_{l+1}-\hat{u}_{l+1}\right)\right\|_{Y} \\
& \leq \frac{1}{2}\left(\sum_{j=0}^{l} \alpha_{j}^{-1}\right)^{-1 / 2}\left\|u_{0}-\hat{u}_{0}\right\|_{U}+\sum_{m=0}^{l} \alpha_{m}^{-1}\left(\sum_{j=m}^{l} \alpha_{j}^{-1}\right)^{-1} \sum_{i=1}^{4}\left\|h_{m}^{(i)}\right\|_{Y} \\
& \leq \frac{1}{2} c_{0} \alpha_{l+1}^{1 / 2}\left\|u_{0}-\hat{u}_{0}\right\|_{U}+C(\rho) \sum_{m=0}^{l} \alpha_{m}^{-1}\left(\sum_{j=m}^{l} \alpha_{j}^{-1}\right)^{-1} \\
& \times\left[\alpha_{m}^{\nu+1 / 2}\|w\|_{U}+\alpha_{m}^{1 / 2}\left\|u_{m}-\hat{u}_{m}\right\|_{U}+\left\|G_{\dagger}\left(u_{m}-\hat{u}_{m}\right)\right\|_{Y}\right] .
\end{aligned}
$$


The induction hypothesis as well as Lemmas A.1 and A.2 then imply that

$$
\begin{aligned}
& \left\|G_{\dagger}\left(u_{l+1}-\hat{u}_{l+1}\right)\right\|_{Y} \\
& \begin{array}{l}
\leq \frac{1}{2} c_{0} \alpha_{l+1}^{1 / 2}\left\|u_{0}-\hat{u}_{0}\right\|_{U} \\
+C(\rho)\left(2+c_{0}\right)\left\|u_{0}-\hat{u}_{0}\right\|_{U} \sum_{m=0}^{l} \alpha_{m}^{-1 / 2}\left(\sum_{j=m}^{l} \alpha_{j}^{-1}\right)^{-1} \\
\quad+C(\rho)\left(1+\pi_{1}(\rho)+\pi_{2}(\rho)\right)\|w\|_{U} \sum_{m=0}^{l} \alpha_{m}^{\nu-1 / 2}\left(\sum_{j=m}^{l} \alpha_{j}^{-1}\right)^{-1} \\
\leq\left\|u_{0}-\hat{u}_{0}\right\|_{U} \alpha_{l+1}^{1 / 2}\left[\frac{1}{2} c_{0}+C(\rho)\left(2+c_{0}\right) c_{2}\right] \\
\quad+C(\rho) K_{1}(r, \nu)\left(1+\pi_{1}(\rho)+\pi_{2}(\rho)\right)\|w\|_{U} \alpha_{l+1}^{\nu+1 / 2}
\end{array} \\
& \leq c_{0}\left\|u_{0}-\hat{u}_{0}\right\|_{U} \alpha_{l+1}^{1 / 2}+2 C(\rho) K_{1}(r, \nu)\|w\|_{U} \alpha_{l+1}^{\nu+1 / 2},
\end{aligned}
$$

where the last inequality follows from (2.62), the definition of $C(\rho)$, and the monotonic growth of $\kappa, \eta$. We thus obtain the desired conclusion.

The following corollary is a direct consequence of Lemmas 2.11 and 2.13.

COROLLARY 2.14. Under the assumptions of Lemma 2.13, it holds that

$$
\left\|e_{n}\right\|_{U} \leq 2\left\|u_{0}-\hat{u}_{0}\right\|_{U}+\left(2 c_{0}+\pi_{1}(\rho)\right) \alpha_{n}^{\nu}\|w\|_{U}
$$

and

$$
\left\|G_{\dagger} e_{n}\right\|_{Y} \leq c_{0} \alpha_{n}^{1 / 2}\left\|u_{0}-\hat{u}_{0}\right\|_{U}+\left(2 c_{0}^{2}+\pi_{2}(\rho)\right) \alpha_{n}^{\nu+1 / 2}\|w\|_{U}
$$

for all $n \geq 0$.

In the remainder of this section, we show the convergence of the sequence $\left\{u_{n}\right\}$ to $u^{\dagger}$.

THEOREM 2.15. Let $\left\{\alpha_{n}\right\}$ be defined by (2.9) and (2.10). Assume that Assumption (A1) holds and that there exists a constant $\bar{\rho}_{2}$ satisfying (2.62) corresponding to $\nu=\frac{1}{4}$. Let $\rho \in\left(0, \bar{\rho}_{2}\right]$ and $u_{0} \in U$ satisfy $4\left\|e_{0}\right\|_{U}<\rho$ and $e_{0} \in \mathcal{N}\left(G_{\dagger}\right)^{\perp}$. Then, it holds that

$$
\left\|e_{n}\right\|_{U} \rightarrow 0 \quad \text { and } \quad \frac{\left\|G_{\dagger} e_{n}\right\|_{Y}}{\sqrt{\alpha_{n}}} \rightarrow 0 \quad \text { as } n \rightarrow \infty .
$$

Proof. Let $\varepsilon>0$ be such that $4 \varepsilon<\rho$. Since $e_{0} \in \mathcal{N}\left(G_{\dagger}\right)^{\perp}$ and

$$
\mathcal{N}\left(G_{\dagger}\right)^{\perp}=\overline{\mathcal{R}\left(G_{\dagger}^{*}\right)}=\overline{\mathcal{R}\left(A^{1 / 2}\right)} \subset \overline{\mathcal{R}\left(A^{1 / 4}\right)},
$$

there exists an element $\hat{u} \in U$ such that $\left\|\hat{u}_{0}-u_{0}\right\|<\varepsilon$ and $\hat{u}_{0}-u^{\dagger}=A^{1 / 4} w$ for some $w \in U$. Obviously, $2\left\|\hat{e}_{0}\right\|_{U}<\rho$ with $\hat{e}_{0}:=\hat{u}_{0}-u^{\dagger}$. Applying Corollary 2.14 to the case $\nu=1 / 4$ leads to the estimates

$$
\left\|e_{n}\right\|_{U} \leq 2\left\|u_{0}-\hat{u}_{0}\right\|_{U}+\left(2 c_{0}+\pi_{1}(\rho)\right) \alpha_{n}^{1 / 4}\|w\|_{U}
$$

and

$$
\left\|G_{\dagger} e_{n}\right\|_{Y} \leq c_{0} \alpha_{n}^{1 / 2}\left\|u_{0}-\hat{u}_{0}\right\|_{U}+\left(2 c_{0}^{2}+\pi_{2}(\rho)\right) \alpha_{n}^{3 / 4}\|w\|_{U} .
$$

Since $\alpha_{n} \rightarrow 0$ as $n \rightarrow \infty$, there exists a number $n_{0}:=n_{0}\left(\varepsilon,\|w\|_{U}\right)$ such that

$$
\left(2 c_{0}+\pi_{1}(\rho)\right) \alpha_{n}^{1 / 4}\|w\|_{U} \leq \varepsilon \quad \text { for all } n \geq n_{0} .
$$

This and (2.68) give $\left\|e_{n}\right\|_{U} \leq 3 \varepsilon$ for all $n \geq n_{0}$. The first limit in (2.67) then follows. The second limit in (2.67) is similarly obtained from (2.69). 
2.3. Asymptotic stability estimates. This section provides estimates for $\left\|u_{n}^{\delta}-u_{n}\right\|_{U}$ and $\left\|G_{\dagger}\left(u_{n}^{\delta}-u_{n}\right)\right\|_{Y}$, with $0 \leq n \leq \tilde{N}_{\delta}$, that are crucial to prove the regularization property of the modified Levenberg-Marquardt method.

PROPOSITION 2.16. Let all assumptions in Lemma 2.13 hold true. Assume furthermore that a positive constant $\rho_{3} \leq \min \left\{\rho_{2}, \bar{\rho}_{2}\right\}$ exists such that

$$
\left\{\begin{array}{l}
T_{1}\left(\rho_{3}\right)+T_{2}\left(\rho_{3}\right) \leq 2+c_{0} \\
\pi_{1}\left(\rho_{3}\right)+\pi_{2}\left(\rho_{3}\right)+T_{1}\left(\rho_{3}\right)+T_{2}\left(\rho_{3}\right) \leq 2 c_{0}\left(1+c_{0}\right), \\
L_{1}\left(\rho_{3}\right)\left(3+c_{3}+T_{3}\left(\rho_{3}\right)\right) \leq 1 \\
T_{3}\left(\rho_{3}\right) \leq 3+c_{3}
\end{array}\right.
$$

with

$$
\begin{aligned}
& T_{1}(\rho):=\left(2 c_{0}+2 c_{0}^{2}\right) K_{0}(r, \nu) L_{1}(\rho), \quad T_{2}(\rho):=4 c_{0}\left(1+c_{0}\right) K_{1}(r, \nu) L_{1}(\rho), \\
& T_{3}(\rho):=2 c_{4}\left(3+c_{3}\right) L_{1}(\rho)
\end{aligned}
$$

$L_{1}(\rho)$ defined as in Lemma 2.7, and $\pi_{1}(\rho)$ and $\pi_{2}(\rho)$ given in Lemma 2.13. Let $\rho \leq \rho_{3}$ and $u_{0}, \hat{u}_{0} \in U$ be such that $\min \left\{2\left\|e_{0}\right\|_{U}, 2\left\|\hat{e}_{0}\right\|_{U}\right\}<\rho$ and $\hat{e}_{0}=A^{\nu} w$ for some $w \in U$. Then it holds that

$$
\left\|u_{n}^{\delta}-u_{n}\right\|_{U} \leq T_{1}(\rho)\left(\left\|u_{0}-\hat{u}_{0}\right\|_{U}+\alpha_{n}^{\nu}\|w\|_{U}\right)+c_{3} \frac{\delta}{\sqrt{\alpha_{n}}}
$$

and

$$
\left\|G_{\dagger}\left(u_{n}^{\delta}-u_{n}\right)\right\|_{Y} \leq T_{2}(\rho)\left(\left\|u_{0}-\hat{u}_{0}\right\|_{U} \alpha_{n}^{1 / 2}+\alpha_{n}^{\nu+1 / 2}\|w\|_{U}\right)+\left(2+T_{3}(\rho)\right) \delta
$$

Proof. We show (2.71) and (2.72) by induction on $0 \leq n \leq \tilde{N}_{\delta}$. It is easy to see that these estimates are valid for $n=0$. Now for any fixed $0 \leq l<\tilde{N}_{\delta}$ we assume that (2.71) and (2.72) are fulfilled for all $0 \leq n \leq l$ and show that these estimates also hold true for $n=l+1$. To this end, using (2.36), the induction hypothesis, and Corollary 2.14, we estimate

$$
\begin{aligned}
\left\|u_{l+1}^{\delta}-u_{l+1}\right\|_{U} \leq \frac{L_{1}(\rho)}{2} & \sum_{m=0}^{l} \alpha_{m}^{-1}\left(\sum_{j=m}^{l} \alpha_{j}^{-1}\right)^{-1 / 2} \\
& \times\left\|u_{0}-\hat{u}_{0}\right\|_{U} \alpha_{m}^{1 / 2}\left(2+c_{0}+T_{1}(\rho)+T_{2}(\rho)\right) \\
& +\frac{L_{1}(\rho)}{2} \sum_{m=0}^{l} \alpha_{m}^{-1}\left(\sum_{j=m}^{l} \alpha_{j}^{-1}\right)^{-1 / 2} \\
& \times\|w\|_{U} \alpha_{m}^{\nu+1 / 2}\left(2 c_{0}+2 c_{0}^{2}+\pi_{1}(\rho)+\pi_{2}(\rho)+T_{1}(\rho)+T_{2}(\rho)\right) \\
+ & \frac{L_{1}(\rho)}{2} \sum_{m=0}^{l} \alpha_{m}^{-1}\left(\sum_{j=m}^{l} \alpha_{j}^{-1}\right)^{-1 / 2} \\
& \times \delta\left(2+c_{3}+T_{3}(\rho)\right)+\frac{1}{2} c_{3}\left(1+L_{1}(\rho)\right) \frac{\delta}{\sqrt{\alpha_{l+1}}},
\end{aligned}
$$


which together with Lemmas A.1 and A.2 leads to

$$
\begin{aligned}
& \left\|u_{l+1}^{\delta}-u_{l+1}\right\|_{U} \\
& \leq \frac{1}{2} L_{1}(\rho)\left\|u_{0}-\hat{u}_{0}\right\|_{U}\left(2+c_{0}+T_{1}(\rho)+T_{2}(\rho)\right) c_{1} \\
& \quad+\frac{1}{2} L_{1}(\rho)\|w\|_{U}\left(2 c_{0}+2 c_{0}^{2}+\pi_{1}(\rho)+\pi_{2}(\rho)+T_{1}(\rho)+T_{2}(\rho)\right) K_{0}(r, \nu) \alpha_{l+1}^{\nu} \\
& \quad+\frac{1}{2} L_{1}(\rho)\left(2+c_{3}+T_{3}(\rho)\right) c_{3} \delta \alpha_{l+1}^{-1 / 2}+\frac{1}{2} c_{3}\left(1+L_{1}(\rho)\right) \frac{\delta}{\sqrt{\alpha_{l+1}}},
\end{aligned}
$$

or, equivalently,

$$
\begin{aligned}
& \left\|u_{l+1}^{\delta}-u_{l+1}\right\|_{U} \\
& \leq \frac{1}{2} c_{3}\left[1+L_{1}(\rho)\left(3+c_{3}+T_{3}(\rho)\right)\right] \frac{\delta}{\sqrt{\alpha_{l+1}}} \\
& \quad+\frac{1}{2} L_{1}(\rho)\left\|u_{0}-\hat{u}_{0}\right\|_{U}\left(2+c_{0}+T_{1}(\rho)+T_{2}(\rho)\right) c_{1} \\
& \quad+\frac{1}{2} L_{1}(\rho)\|w\|_{U}\left(2 c_{0}+2 c_{0}^{2}+\pi_{1}(\rho)+\pi_{2}(\rho)+T_{1}(\rho)+T_{2}(\rho)\right) K_{0}(r, \nu) \alpha_{l+1}^{\nu} .
\end{aligned}
$$

From this and (2.70), the monotonic growth of $\kappa$ and $\eta$ gives

$$
\left\|u_{l+1}^{\delta}-u_{l+1}\right\|_{U} \leq T_{1}(\rho)\left(\left\|u_{0}-\hat{u}_{0}\right\|_{U}+\alpha_{l+1}^{\nu}\|w\|_{U}\right)+c_{3} \frac{\delta}{\sqrt{\alpha_{l+1}}} .
$$

The estimate (2.71) is thus verified for $n=l+1$. Similarly, from (2.37), the induction hypothesis, and Corollary 2.14, we obtain

$$
\begin{aligned}
& \left\|G_{\dagger}\left(u_{l+1}^{\delta}-u_{l+1}\right)-y^{\delta}+y^{\dagger}\right\|_{Y} \\
& \leq L_{1}(\rho) \sum_{m=0}^{l} \alpha_{m}^{-1}\left(\sum_{j=m}^{l} \alpha_{j}^{-1}\right)^{-1}\left\|u_{0}-\hat{u}_{0}\right\|_{U} \alpha_{m}^{1 / 2}\left(2+c_{0}+T_{1}(\rho)+T_{2}(\rho)\right) \\
& \quad+L_{1}(\rho) \sum_{m=0}^{l} \alpha_{m}^{-1}\left(\sum_{j=m}^{l} \alpha_{j}^{-1}\right)^{-1}\|w\|_{U} \alpha_{m}^{\nu+1 / 2} \\
& \quad \times\left(2 c_{0}+2 c_{0}^{2}+\pi_{1}(\rho)+\pi_{2}(\rho)+T_{1}(\rho)+T_{2}(\rho)\right) \\
& \quad+L_{1}(\rho) \sum_{m=0}^{l} \alpha_{m}^{-1}\left(\sum_{j=m}^{l} \alpha_{j}^{-1}\right)^{-1} \delta\left(2+c_{3}+T_{3}(\rho)\right)+\delta\left(1+c_{4} L_{1}(\rho)\right),
\end{aligned}
$$

which together with Lemmas A.1 and A.2 leads to

$$
\begin{aligned}
& \left\|G_{\dagger}\left(u_{l+1}^{\delta}-u_{l+1}\right)-y^{\delta}+y^{\dagger}\right\|_{Y} \\
& \leq \delta \\
& \quad\left[1+L_{1}(\rho) c_{4}\left(3+c_{3}+T_{3}(\rho)\right)\right] \\
& \quad+L_{1}(\rho)\left\|u_{0}-\hat{u}_{0}\right\|_{U}\left(2+c_{0}+T_{1}(\rho)+T_{3}(\rho)\right) c_{2} \alpha_{l+1}^{1 / 2} \\
& \quad+L_{1}(\rho)\|w\|_{U}\left(2 c_{0}+2 c_{0}^{2}+\pi_{1}(\rho)+\pi_{2}(\rho)+T_{1}(\rho)+T_{2}(\rho)\right) K_{1}(r, \nu) \alpha_{l+1}^{\nu+1 / 2} .
\end{aligned}
$$


By virtue of (2.70) and the monotonicity of $\kappa$ and $\eta$, we have that

$$
\begin{aligned}
& \left\|G_{\dagger}\left(u_{l+1}^{\delta}-u_{l+1}\right)-y^{\delta}+y^{\dagger}\right\|_{Y} \\
& \leq L_{1}(\rho)\left\|u_{0}-\hat{u}_{0}\right\|_{U}\left(4+2 c_{0}\right) c_{2} \alpha_{l+1}^{1 / 2}+L_{1}(\rho)\|w\|_{U}\left(4 c_{0}+4 c_{0}^{2}\right) K_{1}(r, \nu) \alpha_{l+1}^{\nu+1 / 2} \\
& \quad+\delta\left[1+2 L_{1}(\rho) c_{4}\left(3+c_{3}\right)\right] \\
& \leq T_{2}(\rho)\left(\left\|u_{0}-\hat{u}_{0}\right\|_{U} \alpha_{l+1}^{1 / 2}+\|w\|_{U} \alpha_{l+1}^{\nu+1 / 2}\right)+\delta\left(1+T_{3}(\rho)\right)
\end{aligned}
$$

Noting that $L_{1}(\rho)\left(4+2 c_{0}\right) c_{2} \leq L_{1}(\rho)\left(4 c_{0}+4 c_{0}^{2}\right) K_{1}(r, \nu)=: T_{2}(\rho)$, the estimate (2.72) is therefore satisfied for $n=l+1$.

As a result of (2.73) and Proposition 2.16, we have the following corollary, whose proof is similar to that of (2.41).

COROLlary 2.17. Let all assumptions of Proposition 2.16 be satisfied. Then it holds that

$\left\|F\left(u_{n}^{\delta}\right)-F\left(u_{n}\right)-y^{\delta}+y^{\dagger}\right\|_{Y} \leq T_{4}(\rho)\left(\left\|u_{0}-\hat{u}_{0}\right\|_{U} \alpha_{n}^{1 / 2}+\|w\|_{U} \alpha_{n}^{\nu+1 / 2}\right)+\delta\left(1+T_{5}(\rho)\right)$

for all $0 \leq n \leq \tilde{N}_{\delta}$, where

$$
\begin{aligned}
& T_{4}(\rho):=T_{2}(\rho)\left(1+\kappa(\rho)+\frac{1+\kappa_{0}}{1-\eta_{0}} \eta(\rho)\right), \\
& T_{5}(\rho):=T_{3}(\rho)+\left(\kappa(\rho)+\frac{1+\kappa_{0}}{1-\eta_{0}} \eta(\rho)\right)\left(2+T_{3}(\rho)\right),
\end{aligned}
$$

and $T_{2}(\rho)$ and $T_{3}(\rho)$ are defined as in Proposition 2.16.

2.4. Regularization property. This section is concerned with the convergence of the sequence $\left\{u_{N_{\delta}}^{\delta}\right\}$ as $\delta \rightarrow 0$ provided that $e_{0} \in \mathcal{N}\left(G_{\dagger}\right)^{\perp}$ and that $u_{0}$ is sufficiently close to $u^{\dagger}$. Let $\left\{\delta_{k}\right\}$ be a positive zero sequence. To simplify the notation, from now on we write $N_{k}:=N_{\delta_{k}}$. The next lemma will be used to show the convergence of subsequences of $\left\{u_{N_{k}}^{\delta_{k}}\right\}$ for the case where $\left\{N_{k}\right\}$ is bounded.

LEMMA 2.18. Assume that all assumptions of Lemma 2.5 are satisfied. Let $\bar{N} \in \mathbb{N}$ be arbitrary but fixed, and let $\left\{\delta_{k}\right\}$ be a positive zero sequence such that $\tilde{N}_{\delta_{k}} \geq \bar{N}$ for all $k \geq 1$. Assume in addition that Assumption (A2) holds. Then for any subsequence of $\left\{\delta_{k}\right\}$ there exist a subsequence $\left\{\delta_{k_{i}}\right\}$ and elements $\tilde{u}_{j} \in \bar{B}_{U}\left(u^{\dagger}, \rho\right)$, for $0 \leq j \leq \bar{N}$, such that

$$
u_{j}^{\delta_{k_{i}}} \rightarrow \tilde{u}_{j} \quad \text { as } i \rightarrow \infty
$$

for all $0 \leq j \leq \bar{N}$.

Proof. We shall show by induction on $j$ the existence of a subsequence $\left\{\delta_{k_{i}}\right\}$ and elements $\tilde{u}_{j} \in \bar{B}_{U}\left(u^{\dagger}, \rho\right)$, for $0 \leq j \leq \bar{N}$, that satisfy (2.74).

First, (2.74) holds for $j=0$ with $\tilde{u}_{0}:=u_{0}$. By a slight abuse of notation, we assume that $\left\{\delta_{k_{i}}\right\}$ itself is a subsequence satisfying $u_{j}^{\delta_{k_{i}}} \rightarrow \tilde{u}_{j}$ as $i \rightarrow \infty$ for some $\tilde{u}_{j} \in \bar{B}_{U}\left(u^{\dagger}, \rho\right)$ and some $0 \leq j<\bar{N}$. To simplify the notation, we write

$$
u_{j}^{(i)}:=u_{j}^{\delta_{k_{i}}}, \quad u_{j+1}^{(i)}:=u_{j+1}^{\delta_{k_{i}}}, \quad A_{j}^{(i)}:=A_{j}^{\delta_{k_{i}}}, \quad \text { and } \quad G_{j}^{(i)}:=G_{j}^{\delta_{k_{i}}} .
$$


It follows from (2.8) and Lemma 2.3 that

$$
\begin{aligned}
u_{j+1}^{(i)}= & u_{j}^{(i)}+\left(\alpha_{j} I+A_{j}^{(i)}\right)^{-1} G_{j}^{(i) *}\left(y^{\delta_{k_{i}}}-F\left(u_{j}^{(i)}\right)\right) \\
= & u_{j}^{(i)}+\left(\alpha_{j} I+A\right)^{-1} G_{\dagger}^{*}\left[I+S_{\alpha_{j}}\left(u_{j}^{(i)}, u^{\dagger}\right)\right]\left(y^{\delta_{k_{i}}}-F\left(u_{j}^{(i)}\right)\right) \\
= & u_{j}^{(i)}+\left(\alpha_{j} I+A\right)^{-1} G_{\dagger}^{*}\left[I+S_{\alpha_{j}}\left(u_{j}^{(i)}, u^{\dagger}\right)\right]\left(y^{\dagger}-F\left(\tilde{u}_{j}\right)\right) \\
& \quad+\left(\alpha_{j} I+A\right)^{-1} G_{\dagger}^{*}\left[I+S_{\alpha_{j}}\left(u_{j}^{(i)}, u^{\dagger}\right)\right]\left(y^{\delta_{k_{i}}}-F\left(u_{j}^{(i)}\right)-y^{\dagger}+F\left(\tilde{u}_{j}\right)\right)
\end{aligned}
$$

and thus

$$
u_{j+1}^{(i)}=u_{j}^{(i)}+a_{i}+b_{i},
$$

where

$$
\begin{aligned}
& a_{i}:=\left(\alpha_{j} I+A\right)^{-1} G_{\dagger}^{*} h_{i}, \\
& b_{i}:=\left(\alpha_{j} I+A\right)^{-1} G_{\dagger}^{*}\left[I+S_{\alpha_{j}}\left(u_{j}^{(i)}, u^{\dagger}\right)\right]\left(y^{\delta_{k_{i}}}-F\left(u_{j}^{(i)}\right)-y^{\dagger}+F\left(\tilde{u}_{j}\right)\right)
\end{aligned}
$$

with

$$
h_{i}:=\left[I+S_{\alpha_{j}}\left(u_{j}^{(i)}, u^{\dagger}\right)\right]\left(y^{\dagger}-F\left(\tilde{u}_{j}\right)\right) .
$$

Applying Lemma A.4 with $m=l$ and using (2.13) gives

$$
\left\|b_{i}\right\|_{U} \leq \frac{1}{2 \sqrt{\alpha_{j}}}(1+3 \kappa(\rho))\left\|y^{\delta_{k_{i}}}-F\left(u_{j}^{(i)}\right)-y^{\dagger}+F\left(\tilde{u}_{j}\right)\right\|_{Y} .
$$

Letting $i \rightarrow \infty$ and employing the continuity of $F$ yields

$$
b_{i} \rightarrow 0 \quad \text { as } i \rightarrow \infty .
$$

Furthermore, (2.13) ensures the boundedness of the sequence $\left\{h_{i}\right\}$ in $Y$. Moreover, as a result of Assumption (A2), the operator $\left(\alpha_{j} I+A\right)^{-1} G_{\dagger}^{*}$ is compact. This implies that $\left\{a_{i}\right\}$ is compact in $U$. There thus exist a subsequence of $\left\{a_{i}\right\}$, denoted by the same symbol, and an element $a \in U$ such that

$$
a_{i} \rightarrow a \quad \text { as } i \rightarrow \infty .
$$

From (2.75), (2.76), (2.77), and the induction hypothesis, we deduce $u_{j+1}^{(i)} \rightarrow \tilde{u}_{j}+a=: \tilde{u}_{j+1}$. Consequently, (2.74) holds for $j+1$. From Lemma 2.5, we have $u_{j+1}^{(i)} \in \bar{B}\left(u^{\dagger}, \rho\right)$ for all $i \geq 0$ and so $\tilde{u}_{j+1} \in \bar{B}\left(u^{\dagger}, \rho\right)$. The proof is complete.

Before presenting our main theorem, we give a result on the asymptotic stability of the modified Levenberg-Marquardt method. The definition of this notion in the following proposition generalizes that in [3, Def. 2.1].

Proposition 2.19. Let Assumptions (A1) and (A2) be fulfilled. Assume that there exists a constant $\rho_{3}$ satisfying (2.70) in Proposition 2.16 corresponding to $\nu=\frac{1}{4}$. Let $\rho \in\left(0, \rho_{3}\right]$ and $u_{0} \in U$ satisfy $e_{0} \in \mathcal{N}\left(G_{\dagger}\right)^{\perp}$ and $2\left(2+c_{1} \gamma_{0}\right)\left\|e_{0}\right\|_{U}<\rho$. Then the modified Levenberg-Marquardt method (2.8)-(2.11) is asymptotically stable in the following sense: For any subsequence of a positive zero sequence $\left\{\delta_{k}\right\}$, there exist a subsequence $\left\{\delta_{k_{i}}\right\}$ and 
elements $\tilde{u}_{n} \in \bar{B}_{U}\left(u^{\dagger}, \rho\right)$ for all $0 \leq n \leq \bar{N}:=\lim _{i \rightarrow \infty} N_{k_{i}}$ (where the last inequality is strict if $\bar{N}=\infty$ ) such that

$$
\lim _{n \rightarrow \bar{N}}\left(\limsup _{i \rightarrow \infty}\left\|u_{n}^{\delta_{k_{i}}}-\tilde{u}_{n}\right\|_{U}\right)=0
$$

and

$$
\tilde{u}_{n} \rightarrow u^{*} \quad \text { as } n \rightarrow \bar{N}
$$

for some $u^{*} \in S_{\rho}\left(u^{\dagger}\right)$.

Proof. Let $\left\{\delta_{k}\right\}$ itself be a subsequence. Since $\left\{N_{k}\right\}$ is a sequence of integers, there exists a subsequence $\left\{N_{k_{i}}\right\}$ such that either it is a constant sequence or it tends to infinity. For the first case where $N_{k_{i}}=\bar{N}$ for some integer $\bar{N}$ and for all $i \geq 0$, Lemma 2.18 and the discrepancy principle (2.11) give the conclusion of the proposition. For the second case where $N_{k_{i}} \rightarrow \infty$, we shall show that the elements $\tilde{u}_{n}:=u_{n}, n \geq 0$, any subsequence $\left\{\delta_{k_{i}}\right\}$, and $u^{*}:=u^{\dagger}$ satisfy (2.78) and (2.79). To this end, we first see that Theorem 2.15 implies (2.79). Let $\varepsilon>0$ be arbitrary small but fixed such that $2\left(2+c_{1} \gamma_{0}\right) \varepsilon \leq \rho$. Since $e_{0} \in \mathcal{N}\left(G_{\dagger}\right)^{\perp}$ and $\mathcal{N}\left(G_{\dagger}\right)^{\perp}=\overline{\mathcal{R}\left(G_{\dagger}^{*}\right)}=\overline{\mathcal{R}\left(A^{1 / 2}\right)} \subset \overline{\mathcal{R}\left(A^{1 / 4}\right)}$, there is an element $\hat{u} \in U$ such that $\left\|\hat{u}_{0}-u_{0}\right\|<\varepsilon$ and $\hat{e}_{0}:=\hat{u}_{0}-u^{\dagger}=A^{1 / 4} w$ for some $w \in U$. Obviously, we have $\left(2+c_{1} \gamma_{0}\right)\left\|\hat{e}_{0}\right\|_{U}<\rho$. From this and the choice of $\rho$, we thus can apply Proposition 2.16 to obtain the estimate

$$
\left\|u_{n}^{\delta_{k_{i}}}-u_{n}\right\|_{U} \leq T_{1}(\rho)\left(\left\|u_{0}-\hat{u}_{0}\right\|_{U}+\alpha_{n}^{1 / 4}\|w\|_{U}\right)+c_{3} \frac{\delta_{k_{i}}}{\sqrt{\alpha_{n}}}
$$

for all $0 \leq n \leq N_{k_{i}}$ and for all $i \geq 0$. By letting $i \rightarrow \infty$ and then $n \rightarrow \infty$, we therefore have

$$
\limsup _{n \rightarrow \infty}\left(\limsup _{i \rightarrow \infty}\left\|u_{n}^{\delta_{k_{i}}}-u_{n}\right\|_{U}\right) \leq T_{1}(\rho) \varepsilon .
$$

The limit (2.78) then follows.

We are now well prepared to derive the main result of the paper, where some lines in the proof follow the ones in [11].

THEOREM 2.20 (regularization property). Let $\left\{\alpha_{n}\right\}$ be defined by (2.9) and (2.10), and let $\left\{\delta_{k}\right\}$ be a positive zero sequence. Assume that Assumptions (A1) and (A2) hold and that $\tau>\tau_{0}>1, \gamma_{0}>\frac{2 c_{0}}{\left(1-\eta_{0}\right)\left(\tau-\tau_{0}\right)}$. Assume further that a constant $\rho_{3} \leq \rho_{0}$ exists and satisfies (2.70) corresponding to $\nu=\frac{1}{4}$ as well as

$$
T_{5}\left(\rho_{3}\right)<\tau-1
$$

with $T_{5}(\rho)$ defined as in Corollary 2.17 .

Let $\rho \in\left(0, \rho_{3}\right]$ and $u_{0} \in U$ satisfy $2\left(2+c_{1} \gamma_{0}\right)\left\|u_{0}-u^{\dagger}\right\|_{U}<\rho$. Then the method (2.8)-(2.11) is well-defined and the integer $N_{\delta_{k}}$ defined by the discrepancy principle (2.11) satisfies

$$
N_{\delta_{k}}=O\left(1+\left|\log \left(\delta_{k}\right)\right|\right) .
$$

Moreover, if $u_{0}-u^{\dagger} \in \mathcal{N}\left(G_{\dagger}\right)^{\perp}$, then

$$
u_{N_{\delta_{k}}}^{\delta_{k}} \rightarrow u^{\dagger} \quad \text { as } k \rightarrow \infty .
$$


Proof. Under the assumptions, the well-posedness of the method follows from Lemma 2.5, while the logarithmic estimate (2.81) is shown in Lemma 2.10. It is therefore sufficient to prove (2.82).

To this end, we first assume that there exists a subsequence $\delta_{k_{i}}$ such that $N_{k_{i}}=\bar{N}$ for all $i \geq 0$. By virtue of Lemma 2.18, there exist a subsequence $\left\{k_{m}\right\}$ of $\left\{k_{i}\right\}$ and elements $\tilde{u}_{j} \in \bar{B}_{U}\left(u^{\dagger}, \rho\right)$, with $j=0,1, \ldots, \bar{N}$, such that

$$
u_{j}^{\delta_{k_{m}}} \rightarrow \tilde{u}_{j} \quad \text { as } m \rightarrow \infty
$$

for all $0 \leq j \leq \bar{N}$. Moreover, from the discrepancy principle (2.11), we obtain

$$
\left\|F\left(u_{\frac{k_{m}}{N}}^{\delta}\right)-y^{\delta_{k_{m}}}\right\|_{Y} \leq \tau \delta_{k_{m}} .
$$

Letting $m \rightarrow \infty$ and using the continuity of $F$ yields

$$
F\left(\tilde{u}_{\bar{N}}\right)=y^{\dagger}
$$

which together with (2.83) yields that

$$
u_{\frac{k_{m}}{N}}^{\delta_{1}} \rightarrow \tilde{u}_{\bar{N}} \quad \text { as } m \rightarrow \infty
$$

with $\tilde{u}_{\bar{N}} \in S_{\rho}\left(u^{\dagger}\right)$. We now show that $\tilde{u}_{\bar{N}}=u^{\dagger}$. According to (2.8), it holds for all $0 \leq n \leq \bar{N}-1$ and $m \geq 0$ that

$$
u_{n+1}^{\delta_{k_{m}}}-u_{n}^{\delta_{k_{m}}}=G_{n}^{\delta_{k_{m}}} *\left(\alpha_{n} I+G_{n}^{\delta_{k_{m}}} G_{n}^{\delta_{k_{m}} *}\right)^{-1}\left(y^{\delta_{k_{m}}}-F\left(u_{n}^{\delta_{k_{m}}}\right)\right) \subset \mathcal{R}\left(G_{n}^{\delta_{k_{m}} *}\right) .
$$

Combining this inclusion with (2.3), we have $u_{n+1}^{\delta_{k_{m}}}-u_{n}^{\delta_{k_{m}}} \subset \mathcal{R}\left(G_{\dagger}^{*}\right) \subset \mathcal{N}\left(G_{\dagger}\right)^{\perp}$ for all $m \geq 0$ and $0 \leq n \leq \bar{N}-1$. Consequently, using $u_{0}-u^{\dagger} \in \mathcal{N}\left(G_{\dagger}\right)^{\perp}$, it then follows that $u_{\bar{N}}^{\delta_{k_{m}}}-u^{\dagger} \subset \overline{\mathcal{R}}\left(G_{\dagger}^{*}\right) \subset \mathcal{N}\left(G_{\dagger}\right)^{\perp}$. From this and the limit (2.84), we find $\tilde{u}_{\bar{N}}-u^{\dagger} \in \mathcal{N}\left(G_{\dagger}\right)^{\perp}$. On the other hand, as a result of (GTCC) and the fact that $u^{\dagger}, \tilde{u}_{\bar{N}} \in S_{\rho}\left(u^{\dagger}\right)$, it holds that $\tilde{u}_{\bar{N}}-u^{\dagger} \in \mathcal{N}\left(G_{\dagger}\right)$. We thus have $\tilde{u}_{\bar{N}}-u^{\dagger} \in \mathcal{N}\left(G_{\dagger}\right) \cap \mathcal{N}\left(G_{\dagger}\right)^{\perp}=\{0\}$. Therefore, a subsequence-subsequence argument allows us to conclude that

$$
u_{\overline{k_{i}}}^{\delta_{N}} \rightarrow u^{\dagger} \quad \text { as } i \rightarrow \infty
$$

We next assume that there exists a subsequence $\delta_{k_{i}}$ such that $N_{k_{i}} \rightarrow \infty$ as $i \rightarrow \infty$. In this case, let $\varepsilon>0$ be arbitrary but fixed such that $0<2\left(2+c_{1} \gamma_{0}\right) \varepsilon<\rho$. Since $e_{0} \in \mathcal{N}\left(G_{\dagger}\right)^{\perp}$ and $\mathcal{N}\left(G_{\dagger}\right)^{\perp}=\overline{\mathcal{R}\left(G_{\dagger}^{*}\right)}=\overline{\mathcal{R}\left(A^{1 / 2}\right)} \subset \overline{\mathcal{R}\left(A^{1 / 4}\right)}$, there exists an element $\hat{u} \in U$ such that $\left\|\hat{u}_{0}-u_{0}\right\|<\varepsilon$ and $\hat{u}_{0}-u^{\dagger}=A^{1 / 4} w$ for some $w \in U$. Easily, $2\left\|\hat{e}_{0}\right\|_{U} \leq\left(2+c_{1} \gamma_{0}\right)\left\|\hat{e}_{0}\right\|_{U}<\rho$ with $\hat{e}_{0}:=\hat{u}_{0}-u^{\dagger}$. From Proposition 2.16 and Corollary 2.17, we have

$$
\left\|u_{j}^{\delta_{k_{i}}}-u_{j}\right\|_{U} \leq T_{1}(\rho)\left(\left\|u_{0}-\hat{u}_{0}\right\|_{U}+\alpha_{j}^{1 / 4}\|w\|_{U}\right)+c_{3} \frac{\delta_{k_{i}}}{\sqrt{\alpha_{j}}}
$$

and

$$
\begin{gathered}
\left\|F\left(u_{j}^{\delta_{k_{i}}}\right)-F\left(u_{j}\right)-y^{\delta_{k_{i}}}+y^{\dagger}\right\|_{Y} \leq T_{4}(\rho)\left(\left\|u_{0}-\hat{u}_{0}\right\|_{U} \alpha_{j}^{1 / 2}+\alpha_{j}^{3 / 4}\|w\|_{U}\right) \\
+\left(1+T_{5}(\rho)\right) \delta_{k_{i}}
\end{gathered}
$$


for all $0 \leq j \leq N_{k_{i}}$ and for all $i \geq 0$. On the other hand, we can conclude from the discrepancy principle (2.11) and the estimate (2.6) for all $0 \leq j<N_{k_{i}}$ that

$$
\begin{aligned}
\tau \delta_{k_{i}} & <\left\|F\left(u_{j}^{\delta_{k_{i}}}\right)-y^{\delta_{k_{i}}}\right\|_{Y} \leq\left\|F\left(u_{j}^{\delta_{k_{i}}}\right)-F\left(u_{j}\right)-y^{\delta_{k_{i}}}+y^{\dagger}\right\|_{Y}+\left\|F\left(u_{j}\right)-y^{\dagger}\right\|_{Y} \\
& \leq\left\|F\left(u_{j}^{\delta_{k_{i}}}\right)-F\left(u_{j}\right)-y^{\delta_{k_{i}}}+y^{\dagger}\right\|_{Y}+\frac{1}{1-\eta_{0}}\left\|G_{\dagger} e_{j}\right\|_{Y} .
\end{aligned}
$$

Combining this with (2.87) yields for all $0 \leq j<N_{k_{i}}$ that

$$
\delta_{k_{i}}\left(\tau-\left(1+T_{5}(\rho)\right)\right) \leq T_{4}(\rho)\left(\left\|u_{0}-\hat{u}_{0}\right\|_{U} \alpha_{j}^{1 / 2}+\alpha_{j}^{3 / 4}\|w\|_{U}\right)+\frac{1}{1-\eta_{0}}\left\|G_{\dagger} e_{j}\right\|_{Y}
$$

and thus

$$
\begin{aligned}
& \left(\tau-\left(1+T_{5}(\rho)\right) \frac{\delta_{k_{i}}}{\sqrt{\alpha_{k_{i}}}}\right. \\
& \quad \leq T_{4}(\rho)\left(\frac{1}{r^{1 / 2}}\left\|u_{0}-\hat{u}_{0}\right\|_{U}+\frac{1}{r^{3 / 4}} \alpha_{N_{k_{i}}}^{1 / 4}\|w\|_{U}\right)+\frac{1}{\sqrt{r}\left(1-\eta_{0}\right)} \frac{\left\|G_{\dagger} e_{N_{k_{i}}-1}\right\|_{Y}}{\sqrt{\alpha_{N_{k_{i}}}-1}} .
\end{aligned}
$$

Letting $i \rightarrow \infty$, employing (2.80), and using the second limit in (2.67) gives

$$
\left(\tau-\left(1+T_{5}(\rho)\right)\right) \limsup _{i \rightarrow \infty} \frac{\delta_{k_{i}}}{\sqrt{\alpha_{N_{k_{i}}}}} \leq \frac{T_{4}(\rho)}{r^{1 / 2}}\left\|u_{0}-\hat{u}_{0}\right\|_{Y} \leq \frac{T_{4}(\rho)}{r^{1 / 2}} \varepsilon .
$$

Noting that $N_{k_{i}} \rightarrow \infty$ as $i \rightarrow \infty$, this implies that

$$
\limsup _{i \rightarrow \infty} \frac{\delta_{k_{i}}}{\sqrt{\alpha_{N_{k_{i}}}}}=0
$$

This and (2.86) yield

$$
\limsup _{i \rightarrow \infty}\left\|u_{N_{k_{i}}}^{\delta_{k_{i}}}-u_{N_{k_{i}}}\right\|_{U} \leq T_{1}(\rho)\left\|u_{0}-\hat{u}_{0}\right\|_{U} \leq T_{1}(\rho) \varepsilon,
$$

and hence, since $\varepsilon>0$ was arbitrary,

$$
\limsup _{i \rightarrow \infty}\left\|u_{N_{k_{i}}}^{\delta_{k_{i}}}-u_{N_{k_{i}}}\right\|_{U}=0
$$

Together with (2.67), this implies that

$$
u_{N_{k_{i}}}^{\delta_{k_{i}}} \rightarrow u^{\dagger} \quad \text { as } i \rightarrow \infty
$$

From this, (2.85), and a subsequence-subsequence argument, we obtain (2.82).

3. Iterative regularization for a non-smooth forward operator. In this section, we study the solution operator to (1.2) based on previous results from [2,3]. In particular, we show that this operator together with one of its Bouligand subderivatives satisfies the assumptions in Section 2. 
3.1. Well-posedness and directional differentiability. Let $\Omega \subset \mathbb{R}^{d}, 2 \leq d \leq 3$, be a bounded domain with Lipschitz boundary $\partial \Omega$. For $u \in L^{2}(\Omega)$, we consider the equation

$$
\left\{\begin{aligned}
-\Delta y+y^{+}=u & \text { in } \Omega, \\
y=0 & \text { on } \partial \Omega,
\end{aligned}\right.
$$

where $y^{+}(x):=\max (y(x), 0)$ for all $x \in \Omega$. From [27, Thm. 4.7], we obtain for each $u \in L^{2}(\Omega)$ a unique weak solution $y_{u}$ belonging to $H_{0}^{1}(\Omega) \cap C(\bar{\Omega})$ and satisfying the a priori estimate

$$
\left\|y_{u}\right\|_{H_{0}^{1}(\Omega)}+\left\|y_{u}\right\|_{C(\bar{\Omega})} \leq c_{\infty}\|u\|_{L^{2}(\Omega)}
$$

for some constant $c_{\infty}>0$ independent of $u$.

Let us denote by $F: L^{2}(\Omega) \rightarrow H_{0}^{1}(\Omega) \cap C(\bar{\Omega}) \hookrightarrow L^{2}(\Omega)$ the solution operator of (3.1). As shown in [3, Prop. 3.1] (see also [2, Prop. 2.1]), $F$ is Lipschitz continuous as a function from $L^{2}(\Omega)$ to $H_{0}^{1}(\Omega) \cap C(\bar{\Omega})$, that is,

$$
\|F(u)-F(v)\|_{H_{0}^{1}(\Omega)}+\|F(u)-F(v)\|_{C(\bar{\Omega})} \leq C_{F}\|u-v\|_{L^{2}(\Omega)}
$$

for all $u, v \in L^{2}(\Omega)$ and for some constant $C_{F}$. Moreover, $F$ is completely continuous as a function from $L^{2}(\Omega)$ to $H_{0}^{1}(\Omega)$ and from $L^{2}(\Omega)$ to itself. However, $F$ is in general not Gâteaux differentiable, but it is Gâteaux differentiable at $u$ if and only if $|\{F(u)=0\}|=0$.

Similarly to [3], we shall use as a replacement for the Fréchet derivative a Bouligand subderivative of $F$ as the operator $G_{u}$ in Section 2. We first define the set of Gâteaux points of $F$ as

$$
D:=\left\{v \in L^{2}(\Omega): F: L^{2}(\Omega) \rightarrow H_{0}^{1}(\Omega) \text { is Gâteaux differentiable in } v\right\} .
$$

Denoting the Gâteaux derivative of $F$ at $u \in D$ by $F^{\prime}(u) \in \mathbb{L}\left(L^{2}(\Omega), H_{0}^{1}(\Omega)\right.$ ), the (strongstrong) Bouligand subdifferential at $u \in L^{2}(\Omega)$ is then defined as

$$
\begin{aligned}
& \partial_{B} F(u):=\left\{G_{u} \in \mathbb{L}\left(L^{2}(\Omega), H_{0}^{1}(\Omega)\right): \text { there exists }\left\{u_{n}\right\}_{n \in \mathbb{N}} \subset D\right. \text { such that } \\
&\left.u_{n} \rightarrow u \text { in } L^{2}(\Omega) \text { and } F^{\prime}\left(u_{n}\right) h \rightarrow G_{u} h \text { in } H_{0}^{1}(\Omega) \text { for all } h \in L^{2}(\Omega)\right\} .
\end{aligned}
$$

We have the following convenient characterization of a specific Bouligand subderivative of $F$.

Proposition 3.1 ([2, Prop. 3.16]). Given $u \in L^{2}(\Omega)$, let $G_{u}: L^{2}(\Omega) \rightarrow H_{0}^{1}(\Omega) \hookrightarrow$ $L^{2}(\Omega)$ be the solution operator mapping $h \in L^{2}(\Omega)$ to the unique solution $\zeta \in H_{0}^{1}(\Omega)$ of

$$
\left\{\begin{aligned}
-\Delta \zeta+\chi_{\left\{y_{u}>0\right\}} \zeta=h & & \text { in } \Omega, \\
\zeta=0 & & \text { on } \partial \Omega,
\end{aligned}\right.
$$

where $y_{u}:=F(u)$. Then $G_{u} \in \partial_{B} F(u)$.

In general, for a given $h \in L^{2}(\Omega)$, the mapping $L^{2}(\Omega) \ni u \mapsto G_{u} h \in L^{2}(\Omega)$ is not continuous (see, e.g., [3, Exam. 3.8]), and the mapping $L^{2}(\Omega) \ni u \mapsto G_{u} \in \mathbb{L}\left(L^{2}(\Omega)\right)$ is thus not continuous.

3.2. Verification of the assumptions. We now verify that the solution mapping for our example together with the mapping $G_{u}$ defined as in Proposition 3.1 satisfies Assumption (A1) as well as that-allowing $\rho$ to be taken sufficiently small— the conditions of Theorems 2.15 and 2.20 are satisfied. We begin with the verification of the generalized tangential cone condition (GTCC). 
Proposition 3.2. Let $\bar{u} \in L^{2}(\Omega), \bar{y}:=F(\bar{u})$, and $\rho>0$. Then it holds that

$$
\left\|F(\hat{u})-F(u)-G_{u}(\hat{u}-u)\right\|_{L^{2}(\Omega)} \leq \eta(\rho)\|F(\hat{u})-F(u)\|_{L^{2}(\Omega)}
$$

for all $u, \hat{u} \in \bar{B}_{L^{2}(\Omega)}(\bar{u}, \rho)$ with

$$
\eta(\rho):=C_{\Omega}\left|\left\{|\bar{y}| \leq C_{F} \rho\right\}\right|^{1 / 14}
$$

for some constant $C_{\Omega}>0$.

Proof. Applying to [3, Lem. 3.9] for $p=\frac{7}{4}$ yields

$$
\left\|F(\hat{u})-F(u)-G_{u}(\hat{u}-u)\right\|_{L^{2}(\Omega)} \leq C_{\Omega} M(u, \hat{u})^{1 / 14}\|F(\hat{u})-F(u)\|_{L^{2}(\Omega)}
$$

for some constant $C_{\Omega}$ and $M(u, \hat{u}):=\left|\left\{y_{u} \leq 0, y_{\hat{u}}>0\right\} \cup\left\{y_{u}>0, y_{\hat{u}} \leq 0\right\}\right|$. According to (3.2), we thus have that

$$
\left\|\bar{y}-y_{u}\right\|_{C(\bar{\Omega})} \leq C_{F}\|\bar{u}-u\|_{L^{2}(\Omega)} \leq C_{F} \rho=: \varepsilon
$$

for all $u \in \bar{B}_{L^{2}(\Omega)}(\bar{u}, \rho)$ and $y_{u}:=F(u)$. This implies, for any $u \in \bar{B}_{L^{2}(\Omega)}(\bar{u}, \rho)$, that

$$
-\varepsilon+y_{u}(x) \leq \bar{y} \leq \varepsilon+y_{u}(x)
$$

for all $x \in \bar{\Omega}$ with $y_{u}:=F(u)$. We then have for any $u, \hat{u} \in \bar{B}_{L^{2}(\Omega)}(\bar{u}, \rho)$ that

$$
\begin{aligned}
& \left\{y_{u}>0, y_{\hat{u}} \leq 0\right\} \subset\{-\varepsilon \leq \bar{y} \leq \varepsilon\}, \\
& \left\{y_{u} \leq 0, y_{\hat{u}}>0\right\} \subset\{-\varepsilon \leq \bar{y} \leq \varepsilon\}
\end{aligned}
$$

with $y_{u}:=F(u)$ and $y_{\hat{u}}:=F(\hat{u})$. It therefore holds that

$$
\left|\chi_{\left\{y_{u}>0\right\}}-\chi_{\left\{y_{\hat{u}}>0\right\}}\right|=\left|\chi_{\left\{y_{u}>0, y_{\hat{u}} \leq 0\right\}}-\chi_{\left\{y_{\hat{u}}>0, y_{u} \leq 0\right\}}\right| \leq \chi_{\{-\varepsilon \leq \bar{y} \leq \varepsilon\}} .
$$

From this, we have

$$
M(u, \hat{u}) \leq|\{|\bar{y}| \leq \varepsilon\}|=\left|\left\{|\bar{y}| \leq C_{F} \rho\right\}\right|,
$$

which together with (3.5) allows us to deduce the desired result.

We next construct, for any $u_{1}, u_{2} \in L^{2}(\Omega)$, a bounded linear operator $Q\left(u_{1}, u_{2}\right)$ : $L^{2}(\Omega) \rightarrow L^{2}(\Omega)$ that satisfies (2.3) and (2.4).

LemmA 3.3. Let $u_{1}, u_{2} \in L^{2}(\Omega)$ be arbitrary, and let $G_{u_{i}}, i=1,2$, be defined as in Proposition 3.1. Then there exists a bounded linear operator $Q\left(u_{1}, u_{2}\right): L^{2}(\Omega) \rightarrow L^{2}(\Omega)$ such that

$$
G_{u_{1}}=Q\left(u_{1}, u_{2}\right) G_{u_{2}}
$$

and

$$
\left\|I-Q\left(u_{1}, u_{2}\right)\right\|_{\mathbb{L}\left(L^{2}(\Omega)\right)} \leq C\left(u_{1}, u_{2}\right),
$$

where

$$
C\left(u_{1}, u_{2}\right):=C_{*}\left\|\chi_{\left\{F\left(u_{1}\right)>0\right\}}-\chi_{\left\{F\left(u_{2}\right)>0\right\}}\right\|_{L^{3}(\Omega)}
$$

with some constant $C_{*}>0$ independent of $u_{1}$ and $u_{2}$. 
Proof. To prove the existence of the bounded linear operator $Q\left(u_{1}, u_{2}\right)$, we first construct this operator on $H_{0}^{1}(\Omega)$ and then extend it to $L^{2}(\Omega)$ by a density argument. To this end, we set $y_{i}:=F\left(u_{i}\right)$ with $i=1,2$. We now define the linear operator $Q\left(u_{1}, u_{2}\right): H_{0}^{1}(\Omega) \rightarrow$ $H_{0}^{1}(\Omega) \hookrightarrow L^{2}(\Omega)$ as follows: for any $v \in H_{0}^{1}(\Omega)$, we set $w:=Q\left(u_{1}, u_{2}\right) v$ defined as the unique solution in $H_{0}^{1}(\Omega)$ to

$$
-\Delta w+\chi_{\left\{y_{1}>0\right\}} w=-\Delta v+\chi_{\left\{y_{2}>0\right\}} v \quad \text { in } \Omega .
$$

We now show that

$$
\left\|Q\left(u_{1}, u_{2}\right) v\right\|_{L^{2}(\Omega)} \leq C\|v\|_{L^{2}(\Omega)} \quad \text { for all } v \in H_{0}^{1}(\Omega)
$$

and for some constant $C$ independent of $v$. First, we have for any $v \in H_{0}^{1}(\Omega)$ that

$$
-\Delta(w-v)+\chi_{\left\{y_{1}>0\right\}}(w-v)=\left[\chi_{\left\{y_{2}>0\right\}}-\chi_{\left\{y_{1}>0\right\}}\right] v \quad \text { in } \Omega .
$$

It follows that

$$
\|w-v\|_{H_{0}^{1}(\Omega)} \leq C\|v\|_{L^{2}(\Omega)}
$$

for some constant $C>0$. This and the continuous embedding $H_{0}^{1}(\Omega) \hookrightarrow L^{2}(\Omega)$ give

$$
\|w-v\|_{L^{2}(\Omega)} \leq C\|v\|_{L^{2}(\Omega)},
$$

which along with the triangle inequality yields (3.9). From the estimate (3.9) and the density of $H_{0}^{1}(\Omega)$ in $L^{2}(\Omega)$, the operator $Q\left(u_{1}, u_{2}\right)$ has a unique continuous extension, also denoted by $Q\left(u_{1}, u_{2}\right)$, from $L^{2}(\Omega)$ to $L^{2}(\Omega)$.

It remains to show (3.7) and (3.8). It is easy to obtain the identity (3.7) from the definition of $Q\left(u_{1}, u_{2}\right)$ and the uniqueness of solutions to (3.3). By density, to prove (3.8) we only need to show that

$$
\left\|v-Q\left(u_{1}, u_{2}\right) v\right\|_{L^{2}(\Omega)} \leq C\left(u_{1}, u_{2}\right)\|v\|_{L^{2}(\Omega)} \quad \text { for all } v \in H_{0}^{1}(\Omega) .
$$

Since $\Omega$ is bounded in $\mathbb{R}^{d}$ with $d \in\{2,3\}$, one has $H_{0}^{1}(\Omega) \hookrightarrow L^{6}(\Omega)$. Testing (3.10) by $w-v$ and exploiting the Hölder inequality yield

$$
\begin{aligned}
\|\nabla(w-v)\|_{L^{2}(\Omega)}^{2} & \leq \int_{\Omega}\left[\chi_{\left\{y_{2}>0\right\}}-\chi_{\left\{y_{1}>0\right\}}\right] v(w-v) d x \\
& \leq\left\|\chi_{\left\{y_{2}>0\right\}}-\chi_{\left\{y_{1}>0\right\}}\right\|_{L^{3}(\Omega)}\|v\|_{L^{2}(\Omega)}\|w-v\|_{L^{6}(\Omega)} .
\end{aligned}
$$

From this and the continuous embedding $H_{0}^{1}(\Omega) \hookrightarrow L^{6}(\Omega)$, we obtain

$$
\|\nabla(w-v)\|_{L^{2}(\Omega)} \leq C\left\|\chi_{\left\{y_{2}>0\right\}}-\chi_{\left\{y_{1}>0\right\}}\right\|_{L^{3}(\Omega)}\|v\|_{L^{2}(\Omega)}
$$

for some constant $C$ independent of $u_{1}$ and $u_{2}$. The Poincaré inequality thus implies that

$$
\|w-v\|_{L^{2}(\Omega)} \leq C_{*}\left\|\chi_{\left\{y_{2}>0\right\}}-\chi_{\left\{y_{1}>0\right\}}\right\|_{L^{3}(\Omega)}\|v\|_{L^{2}(\Omega)},
$$

which is identical to (3.11).

Proposition 3.4. Let $Q: L^{2}(\Omega)^{2} \rightarrow \mathbb{L}\left(L^{2}(\Omega)\right)$ be the mapping defined as in Lemma 3.3, let $\bar{u} \in L^{2}(\Omega)$ be arbitrary, and let $\rho$ be a positive number. Then, for any $u_{1}, u_{2} \in \bar{B}_{L^{2}(\Omega)}(\bar{u}, \rho)$, it holds that

$$
\left\|I-Q\left(u_{1}, u_{2}\right)\right\|_{\mathbb{L}\left(L^{2}(\Omega)\right)} \leq \kappa(\rho),
$$


where

$$
\kappa(\rho):=C_{*}\left|\left\{|\bar{y}| \leq C_{F} \rho\right\}\right|^{1 / 3}
$$

with $\bar{y}:=F(\bar{u})$ and $C_{*}$ defined as in Lemma 3.3.

Proof. Set $\bar{y}=F(\bar{u})$. According to (3.2), we thus have that

$$
\left\|\bar{y}-y_{u}\right\|_{C(\bar{\Omega})} \leq C_{F}\|\bar{u}-u\|_{L^{2}(\Omega)} \leq C_{F} \rho=: \varepsilon
$$

for all $u \in \bar{B}_{L^{2}(\Omega)}(\bar{u}, \rho)$ and $y_{u}:=F(u)$. Similar to (3.6), it holds that

$$
\left|\chi_{\left\{y_{1}>0\right\}}-\chi_{\left\{y_{2}>0\right\}}\right| \leq \chi_{\{-\varepsilon \leq \bar{y} \leq \varepsilon\}}
$$

which together with the definition of $C\left(u_{1}, u_{2}\right)$ yields

$$
C\left(u_{1}, u_{2}\right) \leq C_{*}|\{|\bar{y}| \leq \varepsilon\}|^{1 / 3} .
$$

This and Lemma 3.3 give the desired conclusion.

From (3.4) and (3.12), we immediately obtain that $\kappa(\rho)$ and $\eta(\rho)$ can be made arbitrarily small provided that $|\{\bar{y}=0\}|$ is small enough. In particular, we deduce that (GTCC) holds with the required bound on the constant $\eta(\rho)$.

COROLLARY 3.5. Let functions $\kappa$ and $\eta$ be, respectively, defined by (3.12) and (3.4). Let $\bar{u} \in L^{2}(\Omega)$ be such that $|\{F(\bar{u})=0\}|$ is sufficiently small. Then (2.2) holds.

3.3. Bouligand-Levenberg-Marquardt iteration. The results obtained so far indicate that the solution mapping $F$ of (3.1) and the mapping $u \mapsto G_{u}$ with $G_{u}$ the Bouligand subderivative defined as in Proposition 3.1 satisfy Assumption (A1), provided that $\left|\left\{F\left(u^{\dagger}\right)=0\right\}\right|$ is small enough. We note that in this case $F$ is injective, i.e., $u^{\dagger}$ is the unique solution to (2.1). We can therefore exploit $G_{u}$ in the Levenberg-Marquardt method (2.8)-(2.11) to obtain a convergent Bouligand-Levenberg-Marquardt iteration for the iterative regularization of the non-smooth ill-posed problem $F(u)=y$.

COROLlaRY 3.6. Let $u^{\dagger} \in L^{2}(\Omega)$ be such that $\left|\left\{y^{\dagger}=0\right\}\right|$ is small enough with $y^{\dagger}:=F\left(u^{\dagger}\right)$. Let $\left\{\alpha_{n}\right\}$ be defined by (2.9) with $\alpha_{0}^{1 / 2} \geq\left\|G_{u^{\dagger}}\right\|_{\mathbb{L}\left(L^{2}(\Omega)\right)}$. Then there exists $\rho^{*}>0$ such that for all starting points $u_{0} \in \bar{B}_{L^{2}(\Omega)}\left(u^{\dagger}, \rho^{*}\right)$, the Bouligand-LevenbergMarquardt iteration (2.8) stopped according to the discrepancy principle (2.11) is a well-posed and strongly convergent regularization method.

Proof. Take $U=Y=L^{2}(\Omega)$ and note that $\mathcal{N}\left(G_{u^{\dagger}}\right)=\{0\}$ and so $\mathcal{N}\left(G_{u^{\dagger}}\right)^{\perp}=L^{2}(\Omega)$. Then, Assumption (A1) is satisfied according to Propositions 3.2 and 3.4, Lemma 3.3, and Corollary 3.5. Assumption (A2) follows directly from Proposition 3.1 together with the compactness of the embedding $H_{0}^{1}(\Omega) \hookrightarrow L^{2}(\Omega)$. Finally, the various requirements on the smallness of constants involving $\eta(\rho)$ and $\kappa(\rho)$ are satisfied due to Proposition 3.4. The claim now follows from Theorem 2.20.

We point out that the assumption on the support of $F\left(u^{\dagger}\right)$ does not entail a similar requirement on $F\left(u_{n}^{\delta}\right)$ and that this non-differentiability of $F$ at the iterates is the primary source of difficulty in showing convergence.

To close this section, we comment on the practical implementation of the BouligandLevenberg-Marquardt iteration (2.8) for the non-smooth PDE (3.1). Let $y^{\delta} \in L^{2}(\Omega)$. For any $u_{n}^{\delta} \in L^{2}(\Omega)$, we set $y_{n}^{\delta}:=F\left(u_{n}^{\delta}\right)$ and define the correction step

$$
s_{n}^{\delta}:=\left(\alpha_{n} I+\left(G_{n}^{\delta}\right)^{*} G_{n}^{\delta}\right)^{-1}\left(G_{n}^{\delta}\right)^{*}\left(y^{\delta}-y_{n}^{\delta}\right) .
$$


From this, (2.8) can be rewritten as $u_{n+1}^{\delta}=u_{n}^{\delta}+s_{n}^{\delta}$ with

$$
\alpha_{n} s_{n}^{\delta}=\left(G_{n}^{\delta}\right)^{*}\left(-G_{n}^{\delta} s_{n}^{\delta}+y^{\delta}-y_{n}^{\delta}\right) .
$$

By introducing $z_{n}^{\delta}:=G_{n}^{\delta} s_{n}^{\delta}$ and $b_{n}^{\delta}:=y^{\delta}-y_{n}^{\delta}$, we deduce that $s_{n}^{\delta}$ and $z_{n}^{\delta}$ satisfy

$$
\left\{\begin{array}{lll}
-\Delta z_{n}^{\delta}+\chi_{\left\{y_{n}^{\delta}>0\right\}} z_{n}^{\delta}=s_{n}^{\delta} & \text { in } \Omega, & z_{n}^{\delta}=0 \text { on } \partial \Omega, \\
-\Delta s_{n}^{\delta}+\chi_{\left\{y_{n}^{\delta}>0\right\}} s_{n}^{\delta}=\frac{1}{\alpha_{n}}\left(-z_{n}^{\delta}+b_{n}^{\delta}\right) & \text { in } \Omega, & s_{n}^{\delta}=0 \text { on } \partial \Omega .
\end{array}\right.
$$

A Bouligand-Levenberg-Marquardt step can thus be performed by solving a coupled system of two elliptic equations.

4. Numerical experiments. This section provides numerical results that illustrate the performance of the Bouligand-Levenberg-Marquardt iteration. In the first section, we give a short description of our discretization scheme and the solution of the non-smooth PDE using a semismooth Newton (SSN) method. The second section reports the results of numerical examples.

4.1. Discretization. In the following, we restrict ourselves to the case where $\Omega$ is an open bounded convex polygonal domain in $\mathbb{R}^{2}$. We shall use the standard continuous piecewise linear finite elements (FE) (see, e.g., [5, 17]) to discretize the non-smooth semilinear elliptic equation (3.1) as well as the linear system (3.13). In [2,3], the discrete version of (3.1) as well as its equivalent nonlinear algebraic system were obtained by employing a mass lumping scheme for the non-smooth nonlinearity. We shall use the same technique to discretize the system (3.13). Let $\mathcal{T}_{h}$ stand for the triangulation of $\Omega$ corresponding to the parameter $h$, where $h$ denotes the maximum length of the edges of all the triangles of $\mathcal{T}_{h}$. For each triangulation $\mathcal{T}_{h}$, let $V_{h} \subset H_{0}^{1}(\Omega)$ be the space of piecewise linear finite elements on $\Omega$. We denote by $d_{h}$ and $\left\{\varphi_{j}\right\}_{j=1}^{d_{h}}$, respectively, the dimension and the basis of $V_{h}$ corresponding to the set of nodes $\mathcal{N}_{h}:=\left\{x_{1}, \ldots, x_{d_{h}}\right\}$. For each $T \in \mathcal{T}_{h}$, we write $\bar{T}$ for the closure of $T$ (i.e., the inner sum is over all vertices of the triangle $T$ ).

We first consider the nonlinear equation (3.1). Let $y_{h}$ and $u_{h} \in V_{h}$ be the FE approximations of $y$ and $u$, respectively, with $y$ and $u$ satisfying (3.1). As shown in [2,3], the discrete equation of (3.1) is given by

$$
\begin{aligned}
\int_{\Omega} \nabla y_{h} \cdot \nabla v_{h} d x+\frac{1}{3} \sum_{T \in \mathcal{T}_{h}}|T| \sum_{x_{i} \in \bar{T} \cap \mathcal{N}_{h}} \max \left(0, y_{h}\left(x_{i}\right)\right) v_{h}\left(x_{i}\right) & \\
& =\int_{\Omega} u_{h} v_{h} d x, \quad v_{h} \in V_{h},
\end{aligned}
$$

and its equivalent nonlinear algebraic system is defined as

$$
\mathbf{A} y+\mathbf{D} \max (y, 0)=\mathbf{M} u,
$$

where $\mathbf{A}:=\left(\left(\nabla \varphi_{j}, \nabla \varphi_{i}\right)_{L^{2}(\Omega)}\right)_{i, j=1}^{d_{h}}$ is the stiffness matrix, $\mathbf{M}:=\left(\left(\varphi_{j}, \varphi_{i}\right)_{L^{2}(\Omega)}\right)_{i, j=1}^{d_{h}}$ is the mass matrix, $\mathbf{D}:=\frac{1}{3} \operatorname{diag}\left(\omega_{1}, \ldots, \omega_{d_{h}}\right)$ with $\omega_{i}:=\left|\left\{\varphi_{i} \neq 0\right\}\right|$ is the lumped mass matrix, and $\max (\cdot, 0): \mathbb{R}^{d_{h}} \rightarrow \mathbb{R}^{d_{h}}$ is the componentwise max-function. According to [3], the equation (4.1) is semismooth in $\mathbb{R}^{d_{h}}$ and can be solved via a SSN method. Here, with a slight abuse of notation, we write $y \in \mathbb{R}^{d_{h}}$ and $u \in \mathbb{R}^{d_{h}}$, respectively, instead of $\left(y_{h}\left(x_{i}\right)\right)_{i=1}^{d_{h}}$ and $\left(u_{h}\left(x_{i}\right)\right)_{i=1}^{d_{h}}$. 
We now turn to the system (3.13). According to [5, Sec. 2.5] (see also [28, Sec. 9.1.3]), for a fixed $\delta>0$, the discrete linear system of (3.13) is given by

$$
\left\{\begin{array}{c}
\int_{\Omega} \nabla z_{h} \cdot \nabla v_{h} d x+\frac{1}{3} \sum_{T \in \mathcal{T}_{h}}|T| \sum_{x_{i} \in \bar{T} \cap \mathcal{N}_{h}} \chi_{\left\{y_{n}^{\delta}>0\right\}}\left(x_{i}\right) z_{h}\left(x_{i}\right) v_{h}\left(x_{i}\right)=\int_{\Omega} s_{h} v_{h} d x \\
\int_{\Omega} \nabla s_{h} \cdot \nabla w_{h} d x+\frac{1}{3} \sum_{T \in \mathcal{T}_{h}}|T| \sum_{x_{i} \in \bar{T} \cap \mathcal{N}_{h}} \chi_{\left\{y_{n}^{\delta}>0\right\}}\left(x_{i}\right) s_{h}\left(x_{i}\right) w_{h}\left(x_{i}\right)= \\
-\frac{1}{\alpha_{n}} \int_{\Omega}\left(z_{h}-b_{h}\right) w_{h} d x
\end{array}\right.
$$

for all $v_{h}, w_{h} \in V_{h}$, where $z_{h}, s_{h}$, and $b_{h}$ stand for the FE approximations of $z_{n}^{\delta}, s_{n}^{\delta}$, and $b_{n}^{\delta}$, respectively. By standard computations, the above variational system can be reformulated as

$$
\left\{\begin{array}{l}
\mathbf{A} z+\mathbf{K}_{\mathbf{y}} z=\mathbf{M} s \\
\mathbf{A} s+\mathbf{K}_{\mathbf{y}} s=-\frac{1}{\alpha_{n}} \mathbf{M}(z-b)
\end{array}\right.
$$

with

$$
\mathbf{K}_{\mathbf{y}}=\frac{1}{3} \operatorname{diag}\left(\omega_{i} \chi_{\left\{\mathbf{y}_{i}>0\right\}}\right) \in \mathbb{R}^{d_{h} \times d_{h}}, \quad \mathbf{y}_{i}:=y_{n}^{\delta}\left(x_{i}\right), \quad \text { for all } 1 \leq i \leq d_{h} .
$$

Here, again, we denote the coefficient vectors $\left(z_{h}\left(x_{i}\right)\right)_{i=1}^{d_{h}},\left(s_{h}\left(x_{i}\right)\right)_{i=1}^{d_{h}}$, and $\left(b_{h}\left(x_{i}\right)\right)_{i=1}^{d_{h}}$ by $z \in \mathbb{R}^{d_{h}}, s \in \mathbb{R}^{d_{h}}$, and $b \in \mathbb{R}^{d_{h}}$, respectively. A standard argument shows that (4.2) is uniquely solvable.

4.2. Numerical examples. In this section, we consider $\Omega:=(0,1) \times(0,1) \subset \mathbb{R}^{2}$ and employ a uniform triangular Friedrichs-Keller triangulation with $n_{h} \times n_{h}$ vertices for $n_{h}=512$ unless noted otherwise. A direct sparse solver is used to solve the SSN system (4.1) and the linear system (4.2). The SSN iteration for solving (4.1) is initiated at $y^{0}=0$ and terminated if the active sets $A C^{k}:=\left\{i: y_{i}^{k}>0\right\}$ at two consecutive iterates coincide. The Python implementation used to generate the following results (as well as a Julia implementation) can be downloaded from https://github. com/clason/bouligandlevenbergmarquardt. The timings reported in the following were obtained using an Intel Core i7-7600U CPU $(2.80 \mathrm{GHz})$ and $16 \mathrm{GByte}$ RAM.

As in [3], we choose the exact solution

$$
\begin{aligned}
u^{\dagger}\left(x_{1}, x_{2}\right):=\max & \left(y^{\dagger}\left(x_{1}, x_{2}\right), 0\right) \\
+ & {\left[4 \pi^{2} y^{\dagger}\left(x_{1}, x_{2}\right)\right.} \\
& \left.-2\left(\left(2 x_{1}-1\right)^{2}+2\left(x_{1}-1+\beta\right)\left(x_{1}-\beta\right)\right) \sin \left(2 \pi x_{2}\right)\right] \chi_{[\beta, 1-\beta]}\left(x_{1}\right)
\end{aligned}
$$

where

$$
y^{\dagger}\left(x_{1}, x_{2}\right):=\left[\left(x_{1}-\beta\right)^{2}\left(x_{1}-1+\beta\right)^{2} \sin \left(2 \pi x_{2}\right)\right] \chi_{[\beta, 1-\beta]}\left(x_{1}\right),
$$

for some $\beta \in[0,0.5]$, is the corresponding exact state. Obviously, $y^{\dagger} \in H^{2}(\Omega) \cap H_{0}^{1}(\Omega)$ and satisfies (3.1) for the right-hand side $u^{\dagger}$. Moreover, $y^{\dagger}$ vanishes on a set of measure $2 \beta$. The forward operator $F: L^{2}(\Omega) \rightarrow L^{2}(\Omega)$ is therefore not Gâteaux differentiable at $u^{\dagger}$ 
whenever $\beta \in(0,0.5]$; see, e.g., [3, Prop. 3.4]. Let us denote by $y_{h}^{\dagger}$ the discrete projection of $y^{\dagger}$ to $V_{h}$. We now add a random Gaussian noise componentwise to $y_{h}^{\dagger}$ to create noisy data $y_{h}^{\delta}$ corresponding to the noise level

$$
\delta:=\left\|y_{h}^{\dagger}-y_{h}^{\delta}\right\|_{L^{2}(\Omega)} .
$$

Here and below, all norms for the discrete functions $v_{h}$ are computed exactly by $\left\|v_{h}\right\|_{L^{2}(\Omega)}^{2}=v_{h}^{T} \mathbf{M} v_{h}$ (identifying again the function $v_{h}$ with its vector of expansion coefficients). From now on, to simplify the notation, we omit the subscript $h$. In the following, we consider different choices of the parameter $\beta$ and two different choices of starting points: the trivial point $u_{0} \equiv 0$ and the discrete projection of

$$
\bar{u}:=u^{\dagger}-20 \sin \left(\pi x_{1}\right) \sin \left(2 \pi x_{2}\right) .
$$

We point out that for the second starting point, $u^{\dagger}$ satisfies the generalized source condition

$$
u^{\dagger}-\bar{u} \in \mathcal{R}\left[\left(G_{u^{\dagger}}^{*} G_{u^{\dagger}}\right)^{1 / 2}\right] \subset \mathcal{R}\left[\left(G_{u^{\dagger}}^{*} G_{u^{\dagger}}\right)^{\nu}\right]
$$

for some $\nu \in(0,1 / 2)$. Note also that $\bar{u}$ is far from the exact solution $u^{\dagger}$ and that $u_{0} \equiv 0$ is not close to $u^{\dagger}$ when the parameter $\beta$ is far from 0.5. For the case $\beta=0.005$, the exact solution $u^{\dagger}$ and the starting point $\bar{u}$ are displayed in Figure 4.1 and Figure 4.2, respectively. The corresponding noisy data $y^{\delta}$ and the reconstructions $u_{N_{\delta}}^{\delta}$ with respect to the noise level $\delta \in\left\{1.056 \cdot 10^{-2}, 1.058 \cdot 10^{-4}\right\}$ are presented in Figure 4.3 for the parameters $\alpha_{0}=1$, $r=0.5, \beta=0.005, \tau=1.5$, and for the starting point $u_{0}=\bar{u}$.

We now address the regularization property of the Bouligand-Levenberg-Marquardt iteration from Corollary 3.6. We first illustrate the effects of the starting guess on the convergence of the iteration. Table 4.1 displays for the same parameters $\alpha_{0}=1, r=0.5, \beta=0.005$, $\tau=1.5$, a decreasing sequence of noise levels, and both starting points (for the same realization of the random data) the values of the stopping index $N_{\delta}=N\left(\delta, y^{\delta}\right)$, the logarithmic rate of the stopping index

$$
L R_{\delta}:=\frac{N_{\delta}}{1+|\log (\delta)|}
$$

the relative error

$$
E^{\delta}:=\frac{\left\|u^{\dagger}-u_{N_{\delta}}^{\delta}\right\|_{L^{2}(\Omega)}}{\left\|u^{\dagger}\right\|_{L^{2}(\Omega)}},
$$

the empirical convergence rate

$$
R^{\delta}:=\frac{\left\|u^{\dagger}-u_{N_{\delta}}^{\delta}\right\|_{L^{2}(\Omega)}}{\sqrt{\delta}}
$$

as well as the final Tikhonov parameter $\alpha_{N_{\delta}}$ from (1.7). This table indicates that the speed of convergence of the iteration for the starting point $u_{0}=\bar{u}$ is faster than that for the trivial starting point $u_{0} \equiv 0$. While the growth of the stopping index $N_{\delta}$ for the trivial starting point is slightly faster than that for $\bar{u}$, the logarithmic rates (4.3) for both starting points are stable. This fits with Theorem 2.20. For the starting guess $u_{0}=\bar{u}$, the empirical convergence rate $R^{\delta}$ is not greater than 0.4 as $\delta$ is small enough. This agrees with the convergence rate $\mathcal{O}(\sqrt{\delta})$ expected from the classical source condition $u^{\dagger}-u_{0} \in \mathcal{R}\left[\left(F^{\prime}\left(u^{\dagger}\right)^{*} F^{\prime}\left(u^{\dagger}\right)^{1 / 2}\right]\right.$. 


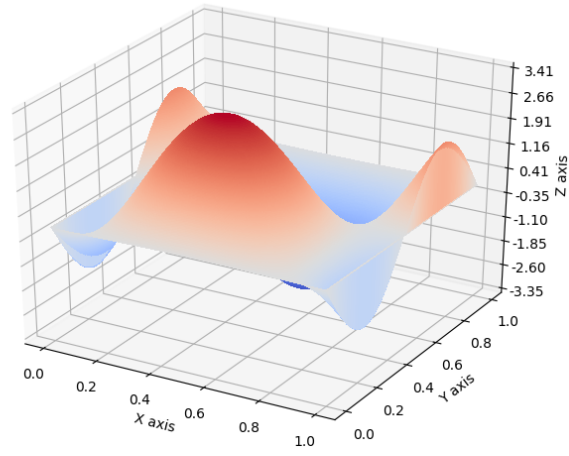

FIG. 4.1. Exact solution $u^{\dagger}$ for $\beta=0.005$.

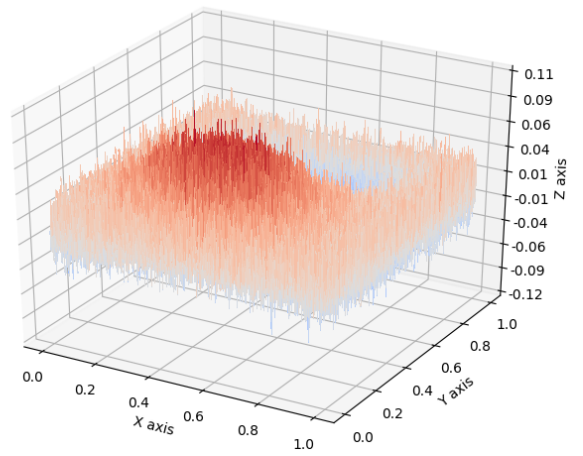

(a) $y^{\delta}, \delta=1.056 \cdot 10^{-2}$.

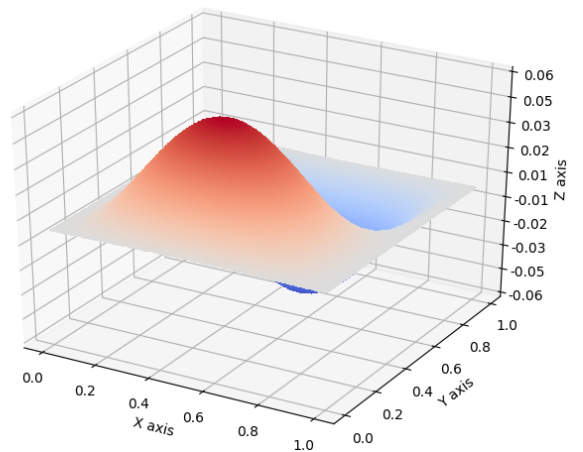

(c) $y^{\delta}, \delta=1.058 \cdot 10^{-4}$.

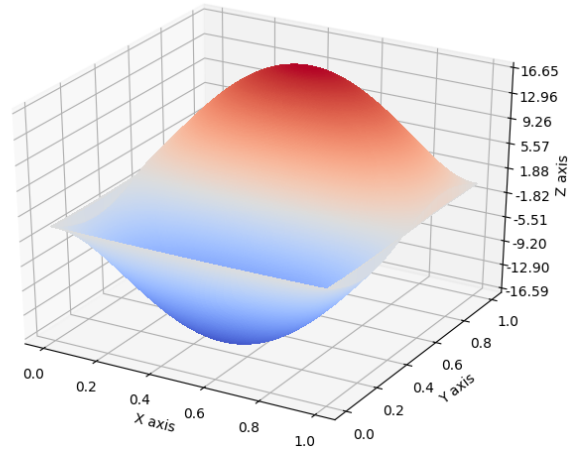

FIG. 4.2. Starting point $u_{0}=\bar{u}$ for $\beta=0.005$.

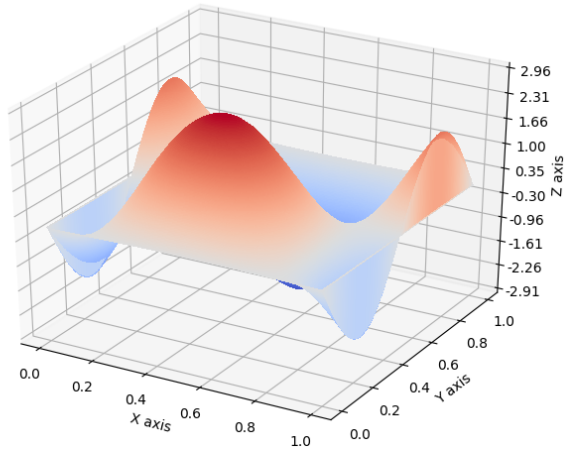

(b) $u_{N_{\delta}}^{\delta}, N_{\delta}=14$.

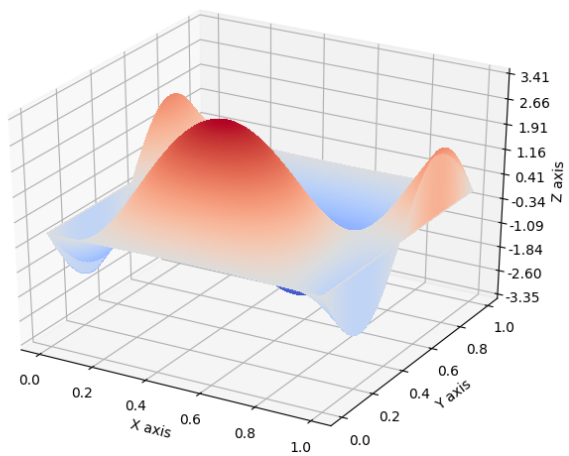

(d) $u_{N_{\delta}}^{\delta}, N_{\delta}=16$.

FIG. 4.3. Noisy data $y^{\delta}$ and reconstructions $u_{N_{\delta}}^{\delta}$ for $u_{0}=\bar{u}$ and $\alpha_{0}=1, r=0.5, \beta=0.005, \tau=1.5$.

To show the dependence on the parameter $\beta$ of the performance of the BouligandLevenberg-Marquardt iteration, we summarize in Table 4.2 the results obtained for $\beta \in$ $\{0,0.15,0.3\}, \alpha_{0}=1, r=0.5, \tau=1.5$, and $u_{0}=\bar{u}$. Table 4.2 indicates that the stopping index does not seem to be significantly influenced by the parameter $\beta$. However, it is not surprising that the relative error $E^{\delta}$ increases with respect to $\beta$ since $\left|\left\{y^{\dagger}=0\right\}\right| \rightarrow 0$ as $\beta \rightarrow 0^{+}$. 


\section{ETNA}

Kent State University and

Johann Radon Institute (RICAM)

TABLE 4.1

Regularization property for $\alpha_{0}=1, r=0.5, \beta=0.005, \tau=1.5$ : noise level $\delta$; stopping index $N_{\delta}$; logarithmic rate $L R_{\delta}$ from (4.3); relative error $E^{\delta}$ from (4.4); empirical convergence rate $R^{\delta}$ from (4.5); final Tikhonov parameter $\alpha_{N_{\delta}}$.

\begin{tabular}{|c|c|c|c|c|c|c|c|c|c|}
\hline \multirow[b]{2}{*}{$\delta$} & \multicolumn{4}{|c|}{$u_{0} \equiv 0$} & \multicolumn{5}{|c|}{$u_{0}=\bar{u}$} \\
\hline & $N_{\delta}$ & $L R_{\delta}$ & $E^{\delta}$ & $\alpha_{N_{\delta}}$ & $N_{\delta}$ & $L R_{\delta}$ & $E^{\delta}$ & $R^{\delta}$ & $\alpha_{N_{\delta}}$ \\
\hline $1.06 \cdot 10^{-2}$ & 12 & 2.2 & $4.70 \cdot 10^{-1}$ & $4.1 \cdot 10^{-9}$ & 14 & 2.5 & $1.55 \cdot 10^{-1}$ & 2.3 & $1.6 \cdot 10^{-10}$ \\
\hline $1.06 \cdot 10^{-3}$ & 16 & 2.0 & $2.36 \cdot 10^{-1}$ & $6.6 \cdot 10^{-12}$ & 15 & 1.9 & $2.07 \cdot 10^{-2}$ & 1.0 & $3.3 \cdot 10^{-11}$ \\
\hline $1.06 \cdot 10^{-4}$ & 20 & 2.0 & $1.44 \cdot 10^{-1}$ & $1.0 \cdot 10^{-14}$ & 16 & 1.6 & $1.57 \cdot 10^{-3}$ & 0.2 & $6.6 \cdot 10^{-12}$ \\
\hline $1.05 \cdot 10^{-5}$ & 25 & 2.0 & $7.33 \cdot 10^{-2}$ & $3.4 \cdot 10^{-18}$ & 17 & 1.4 & $3.57 \cdot 10^{-4}$ & 0.2 & $1.3 \cdot 10^{-12}$ \\
\hline $1.06 \cdot 10^{-6}$ & 30 & 2.0 & $3.55 \cdot 10^{-2}$ & $1.1 \cdot 10^{-21}$ & 18 & 1.2 & $1.85 \cdot 10^{-4}$ & 0.3 & $2.6 \cdot 10^{-13}$ \\
\hline $1.06 \cdot 10^{-7}$ & 34 & 2.0 & $2.77 \cdot 10^{-2}$ & $1.7 \cdot 10^{-24}$ & 21 & 1.2 & $6.47 \cdot 10^{-5}$ & 0.3 & $2.1 \cdot 10^{-15}$ \\
\hline
\end{tabular}

TABLE 4.2

Regularization property for $\alpha_{0}=1, r=0.5, \tau=1.5, u_{0}=\bar{u}$ : noise level $\delta$; stopping index $N_{\delta}=N\left(\delta, y^{\delta}\right)$; relative error $E^{\delta}$ from (4.4).

\begin{tabular}{|c|c|c|c|c|c|c|c|}
\hline \multirow{2}{*}{\multicolumn{2}{|c|}{$\delta$}} & \multicolumn{2}{|r|}{$\beta=0$} & \multicolumn{2}{|c|}{$\beta=0.15$} & \multicolumn{2}{|r|}{$\beta=0.3$} \\
\hline & & $N_{\delta}$ & $E^{\delta}$ & $N_{\delta}$ & $E^{\delta}$ & $\overline{N_{\delta}}$ & $E^{\delta}$ \\
\hline 1.06 & $10^{-1}$ & 11 & 3.10 & 11 & $1.13 \cdot 10^{1}$ & 11 & $6.41 \cdot 10^{1}$ \\
\hline 1.06 & $10^{-2}$ & 14 & $1.50 \cdot 10^{-1}$ & 14 & $5.47 \cdot 10^{-1}$ & 14 & 3.10 \\
\hline 1.06 & $10^{-3}$ & 15 & $1.96 \cdot 10^{-2}$ & 15 & $7.11 \cdot 10^{-2}$ & 15 & $4.11 \cdot 10^{-1}$ \\
\hline 1.06 & $10^{-4}$ & 16 & $1.50 \cdot 10^{-3}$ & 16 & $5.75 \cdot 10^{-3}$ & 16 & $3.52 \cdot 10^{-2}$ \\
\hline 1.06 & $10^{-5}$ & 17 & $3.43 \cdot 10^{-4}$ & 17 & $3.56 \cdot 10^{-3}$ & 17 & $1.02 \cdot 10^{-2}$ \\
\hline 1.06 & $10^{-6}$ & 18 & $1.81 \cdot 10^{-4}$ & 19 & $3.74 \cdot 10^{-3}$ & 18 & $5.29 \cdot 10^{-3}$ \\
\hline
\end{tabular}

Finally, the stopping index as well as the total CPU time (in seconds) of the proposed Bouligand-Levenberg-Marquardt (BLM) iteration and of the Bouligand-Landweber (BL) iteration from [3] are compared in Figure 4.4. Recall that the BL iteration is defined by

$$
u_{n+1}^{\delta}=u_{n}^{\delta}+w_{n} G_{u_{n}^{\delta}}^{*}\left(y^{\delta}-F\left(u_{n}^{\delta}\right)\right), \quad n \geq 0
$$

with the parameter $w_{n}>0$ and is terminated via the discrepancy principle (2.11). To compare the numerical results, we set $\alpha_{0}=1, r=0.5, \beta=0.005, \tau=1.5, w_{n}=(2-2 \mu) / \bar{L}^{2}$ for all $n \geq 0$ with $\mu=0.1$ and $\bar{L}=0.05$. Figure 4.4 displays the stopping index of the two iterative methods versus the noise level $\delta$ for both $u_{0}=\bar{u}$ and $u_{0} \equiv 0$. Figures $4.4 \mathrm{c}$ and $4.4 \mathrm{a}$ indicate that for the BLM iteration, in both cases $N_{\delta}=O(1+|\log (\delta)|)$ as $\delta \rightarrow 0$, as expected from Theorem 2.20. On the other hand, Figures $4.4 \mathrm{~d}$ and $4.4 \mathrm{~b}$ show that for the BL iteration, $N_{\delta}=O\left(\delta^{-1}\right)$ for $u_{0}=\bar{u}$ and $N_{\delta}=O\left(\delta^{-2}\right)$ for $u_{0} \equiv 0$ as $\delta \rightarrow 0$. As also shown in these figures, the total CPU time to run each method is almost directly proportional to their stopping indices (approximately 52 seconds per step for the BLM iteration and 16 seconds per step for the BL iteration, corresponding to the size of (4.2) compared to that of the discretization of (4.6)). For $u_{0} \equiv 0$ and $\delta \approx 5 \cdot 10^{-5}$, the total CPU time of the BLM iteration is only 1136 seconds while that of the BL iteration is nearly 41337 seconds. Similarly, for $u_{0}=\bar{u}$ and $\delta \approx 10^{-7}$ it takes 1087 seconds for the BLM iteration and approximately 18054 seconds for the BL iteration to terminate. Hence even though the cost of each step of the two iterations is different, the BLM iteration is significantly faster also in terms of CPU time for small values of $\delta$. 


\section{ETNA}

Kent State University and Johann Radon Institute (RICAM)

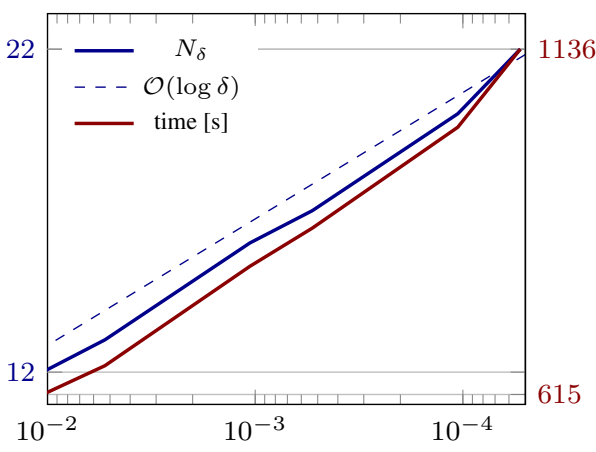

(a) $u_{0}=0$, BLM.

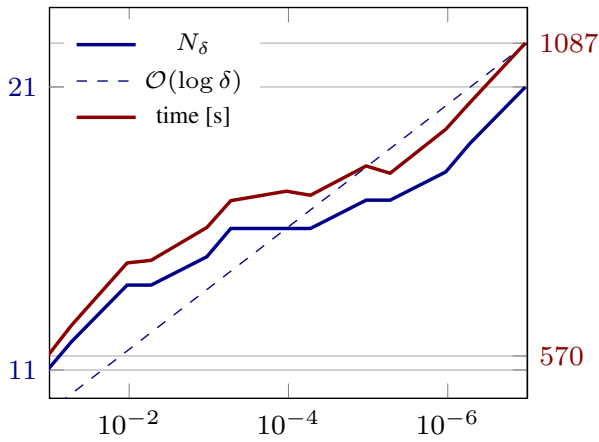

(c) $u_{0}=\bar{u}$, BLM.

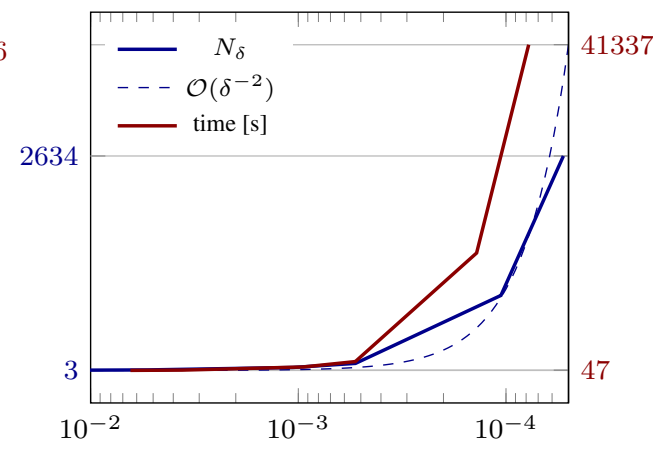

(b) $u_{0}=0, \mathrm{BL}$.

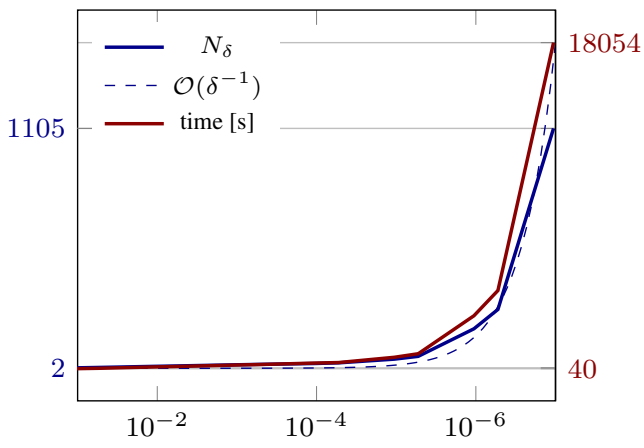

(d) $u_{0}=\bar{u}$, BL.

FIG. 4.4. Comparison of the stopping index $N_{\delta}$ and the total CPU time (in seconds) for the BouligandLevenberg-Marquardt (BLM) and the Bouligand-Landweber (BL) iterations.

5. Conclusion. We have proposed a novel Newton-type regularization method for nonsmooth ill-posed inverse problems that extends the classical Levenberg-Marquardt iteration. Using a family of bounded operators $\left\{G_{u}\right\}$ to replace the Fréchet derivative in the classical Levenberg-Marquardt iteration, we have proved under a generalized tangential cone condition the asymptotic stability of the iterates and from this derived the regularization property of the iteration. In particular, when considering ill-posed inverse problem where the forward operator corresponds to the solution of a non-smooth semilinear elliptic PDE, we can take $G_{u}$ from the Bouligand subdifferential of the forward operator. If the non-differentiability of the forward mapping is sufficiently "weak" at the exact solution, then these operators satisfy the required assumptions and the resulting Bouligand-Levenberg-Marquardt iteration thus provides a convergent regularization method. As the numerical example illustrates, this iteration requires significantly less iterations and can be much faster than first-order methods such as the Bouligand-Landweber iteration from [3].

This work can be extended in several directions. First, it would be interesting to derive convergence rates under the generalized source condition (2.44). Of particular interest would be the extension of the proposed iteration for non-smooth ill-posed inverse problems with additional constraints such as non-negativity of the unknown parameter. Finally, similar non-smooth extensions of other Newton-type methods such as the iteratively regularized Gauss-Newton method could be derived. 
Acknowledgments. This work was supported by the DFG under the grants CL 487/2-1 and RO 2462/6-1, both within the priority program SPP 1962 "Non-smooth and Complementarity-based Distributed Parameter Systems: Simulation and Hierarchical Optimization". The authors would like to thank the two anonymous reviewers for their constructive comments which led to notable improvements of the paper.

Appendix A. Auxiliary lemmas.

This section provides some estimates on the sequence of parameters defined by (2.9) and on bounded linear operators between Hilbert spaces.

LEMMA A.1 ([8]). Let $\left\{\alpha_{n}\right\}$ be defined via (2.9). Then it holds for all $k \geq 0$

$$
\begin{aligned}
\left(\sum_{j=0}^{k} \alpha_{j}^{-1}\right)^{-1} \leq c_{0}^{2} \alpha_{k+1}, & \sum_{m=0}^{k} \alpha_{m}^{-1 / 2}\left(\sum_{j=m}^{k} \alpha_{j}^{-1}\right)^{-1 / 2} \leq c_{1}, \\
\sum_{m=0}^{k} \alpha_{m}^{-1 / 2}\left(\sum_{j=m}^{k} \alpha_{j}^{-1}\right)^{-1} \leq c_{2} \alpha_{k+1}^{1 / 2}, & \sum_{m=0}^{k} \alpha_{m}^{-1}\left(\sum_{j=m}^{k} \alpha_{j}^{-1}\right)^{-1 / 2} \leq c_{3} \alpha_{k+1}^{-1 / 2}, \\
& \sum_{m=0}^{k} \alpha_{m}^{-1}\left(\sum_{j=m}^{k} \alpha_{j}^{-1}\right)^{-1} \leq c_{4},
\end{aligned}
$$

where the constants $c_{i}, i=0, \ldots, 4$, are defined in (2.14).

The next lemma provides some more estimates for the sequence $\left\{\alpha_{n}\right\}$ defined via (2.9) with an exponent $\nu \in[0,1 / 2)$. Its proof is standard and thus is omitted. $k \geq 0$

LEMmA A.2. Let $\left\{\alpha_{n}\right\}$ be defined via (2.9), and let $0 \leq \nu<\frac{1}{2}$. Then it holds for all

$$
\sum_{m=0}^{k} \alpha_{m}^{\nu-1 / 2}\left(\sum_{j=m}^{k} \alpha_{j}^{-1}\right)^{-1 / 2} \leq K_{0}(r, \nu) \alpha_{k+1}^{\nu}
$$

and

$$
\sum_{m=0}^{k} \alpha_{m}^{\nu-1 / 2}\left(\sum_{j=m}^{k} \alpha_{j}^{-1}\right)^{-1} \leq K_{1}(r, \nu) \alpha_{k+1}^{\nu+1 / 2}
$$

with $K_{0}(r, \nu)$ and $K_{1}(r, \nu)$ defined in (2.15).

The next lemmas give useful estimates of a bounded linear operator between Hilbert spaces and generalize the corresponding results in [7]. Their proofs are based on spectral theory and the functional calculus of self-adjoint operators; see, e.g., [4, 26].

Lemma A.3 ([8, Lem. 2]). Let $\left\{\alpha_{k}\right\}$ be a sequence of positive numbers, and let $T: H_{1} \rightarrow H_{2}$ be a bounded linear operator between Hilbert spaces. Then, for any $0 \leq \nu \leq 1$ and any integers $0 \leq m \leq l$, it holds that

$$
\left\|\prod_{j=m}^{l} \alpha_{j}\left(\alpha_{j} I+T^{*} T\right)^{-1}\left(T^{*} T\right)^{\nu}\right\|_{\mathbb{L}\left(H_{1}\right)} \leq\left(\sum_{j=m}^{l} \alpha_{j}^{-1}\right)^{-\nu} .
$$

This result can be improved for the specific case $\nu=\frac{1}{2}$ to be sharp, as can be verified by the choice $m=l$. 
LEMmA A.4. Let $\left\{\alpha_{k}\right\}$ be a sequence of positive numbers, and let $T: H_{1} \rightarrow H_{2}$ be a bounded linear operator between Hilbert spaces. Then, for any integers $0 \leq m \leq l$, it holds that

$$
\left\|\prod_{j=m}^{l} \alpha_{j}\left(\alpha_{j} I+T^{*} T\right)^{-1} T^{*}\right\|_{\mathbb{L}\left(H_{2}, H_{1}\right)} \leq \frac{1}{2}\left(\sum_{j=m}^{l} \alpha_{j}^{-1}\right)^{-1 / 2} .
$$

Proof. Set $t_{0}:=\|T\|_{\mathbb{L}\left(H_{1}, H_{2}\right)}^{2}$ and define the continuous function

$$
g(t):=\prod_{j=m}^{l} \alpha_{j}\left(\alpha_{j}+t\right)^{-1}, \quad t \geq 0
$$

From the spectral theory of self-adjoint operators (see, e.g., [4, Chap. 2]) we have that

$$
\left\|g\left(T^{*} T\right) T^{*}\right\|_{\mathbb{L}\left(H_{2}, H_{1}\right)} \leq \sup \left\{\sqrt{t}|g(t)|: 0 \leq t \leq t_{0}\right\} .
$$

On the other hand, a simple computation and the Cauchy-Schwarz inequality give

$$
\prod_{j=m}^{l}\left(\alpha_{j}+t\right) \geq \prod_{j=m}^{l} \alpha_{j}\left(1+t \sum_{j=m}^{l} \alpha_{j}^{-1}\right) \geq 2 \sqrt{t} \prod_{j=m}^{l} \alpha_{j}\left(\sum_{j=m}^{l} \alpha_{j}^{-1}\right)^{1 / 2},
$$

which leads to

$$
\sqrt{t}|g(t)| \leq \frac{1}{2}\left(\sum_{j=m}^{l} \alpha_{j}^{-1}\right)^{-1 / 2}
$$

for all $t \geq 0$. Combining this with (A.1) yields the desired estimate.

Finally, we have the following direct consequence of Lemmas A.3 and A.4.

COROLLARY A.5. Let $\left\{\alpha_{k}\right\}$ be a sequence of positive numbers, and let $T: H_{1} \rightarrow H_{2}$ be a bounded linear operator between Hilbert spaces. Then, for any $0 \leq \nu \leq \frac{1}{2}$ and any integers $0 \leq m \leq l$, it holds that

$$
\left\|\prod_{j=m}^{l} \alpha_{j}\left(\alpha_{j} I+T^{*} T\right)^{-1}\left(T^{*} T\right)^{\nu} T^{*}\right\|_{\mathbb{L}\left(H_{2}, H_{1}\right)} \leq\left(\sum_{j=m}^{l} \alpha_{j}^{-1}\right)^{-\nu-1 / 2} .
$$

\section{REFERENCES}

[1] M. BRILl AND E. SCHOCK, Iterative solution of ill-posed problems-a survey, in Model Optimization in Exploration Geophysics (Berlin, 1986), A. Vogel, ed., vol. 1 of Theory Practice Appl. Geophys., Vieweg, Braunschweig, 1987, pp. 13-37.

[2] C. Christof, C. Meyer, S. Walther, and C. Clason, Optimal control of a non-smooth semilinear elliptic equation, Math. Control Relat. Fields, 8 (2018), pp. 247-276.

[3] C. Clason AND V. H. NhU, Bouligand-Landweber iteration for a non-smooth ill-posed problem, Numer. Math., 142 (2019), pp. 789-832.

[4] H. W. Engl, M. Hanke, AND A. Neubauer, Regularization of Inverse Problems, Kluwer, Dordrecht, 1996.

[5] R. GLowins KI, Numerical Methods for Nonlinear Variational Problems, Springer, New York, 1984.

[6] M. HANKE, A regularizing Levenberg-Marquardt scheme, with applications to inverse groundwater filtration problems, Inverse Problems, 13 (1997), pp. 79-95. 


\section{ETNA}

Kent State University and

Johann Radon Institute (RICAM)

[7] M. Hanke And C. W. Groetsch, Nonstationary iterated Tikhonov regularization, J. Optim. Theory Appl., 98 (1998), pp. 37-53.

[8] Q. JIN, On a regularized Levenberg-Marquardt method for solving nonlinear inverse problems, Numer. Math., 115 (2010), pp. 229-259.

[9] - Inexact Newton-Landweber iteration for solving nonlinear inverse problems in Banach spaces, Inverse Problems, 28 (2012), Art. 065002, 15 pages.

[10] _ Inexact Newton-Landweber iteration in Banach spaces with nonsmooth convex penalty terms, SIAM J. Numer. Anal., 53 (2015), pp. 2389-2413.

[11] _ Hanke-Raus heuristic rule for variational regularization in Banach spaces, Inverse Problems, 32 (2016), Art. 085008, 19 pages.

[12] Q. JIN AND H. YANG, Levenberg-Marquardt method in Banach spaces with general convex regularization terms, Numer. Math., 133 (2016), pp. 655-684.

[13] Q. JIN AND M. ZHONG, On the iteratively regularized Gauss-Newton method in Banach spaces with applications to parameter identification problems, Numer. Math., 124 (2013), pp. 647-683.

[14] B. KAltenBACher AND B. HofmAnn, Convergence rates for the iteratively regularized Gauss-Newton method in Banach spaces, Inverse Problems, 26 (2010), Art. 035007, 21 pages.

[15] B. Kaltenbacher, A. Neubauer, AND O. SCherZer, Iterative Regularization Methods for Nonlinear Ill-Posed Problems, de Gruyter, Berlin, 2008.

[16] B. KALTENBACHER, F. SCHÖPFER, AND T. SCHUSTER, Iterative methods for nonlinear ill-posed problems in Banach spaces: convergence and applications to parameter identification problems, Inverse Problems, 25 (2009), Art. 065003, 19 pages.

[17] P. Knabner and L. Angermann, Numerical Methods for Elliptic and Parabolic Partial Differential Equations, Springer, New York, 2003.

[18] A. LECHLEITER AND A. RIEDER, Towards a general convergence theory for inexact Newton regularizations, Numer. Math., 114 (2010), pp. 521-548.

[19] F. MARGOTTI AND A. RIEDER, An inexact Newton regularization in Banach spaces based on the nonstationary iterated Tikhonov method, J. Inverse Ill-Posed Probl., 23 (2015), pp. 373-392.

[20] F. MARGOtTI, A. RIEDER, AND A. LeITÃO, A Kaczmarz version of the reg inn-Landweber iteration for ill-posed problems in Banach spaces, SIAM J. Numer. Anal., 52 (2014), pp. 1439-1465.

[21] A. RIEDER, On the regularization of nonlinear ill-posed problems via inexact Newton iterations, Inverse Problems, 15 (1999), pp. 309-327.

[22] — On convergence rates of inexact Newton regularizations, Numer. Math., 88 (2001), pp. 347-365.

[23] - Inexact Newton regularization using conjugate gradients as inner iteration, SIAM J. Numer. Anal., 43 (2005), pp. 604-622.

[24] O. SCHERZER, Convergence criteria of iterative methods based on Landweber iteration for solving nonlinear problems, J. Math. Anal. Appl., 194 (1995), pp. 911-933.

[25] — ed., Handbook of Mathematical Methods in Imaging. Vol. 1, 2, 3, 2nd ed., Springer, New York, 2015.

[26] K. SCHMÜDGEn, Unbounded Self-Adjoint Operators on Hilbert Space, Springer, Dordrecht, 2012.

[27] F. TRÖltzsch, Optimal Control of Partial Differential Equations, American Mathematical Society, Providence, 2010.

[28] M. Ulbrich, Semismooth Newton Methods for Variational Inequalities and Constrained Optimization Problems in Function Spaces, Society for Industrial and Applied Mathematics (SIAM), Philadelphia, 2011. 